\title{
REPRESENTATIONS OF SEMISIMPLE LIE GROUPS. II
}

\author{
BY \\ HARISH-CHANDRA
}

In an earlier paper [5] we have established a close relationship between an irreducible representation of a semisimple Lie group on a Banach space and the corresponding representation of its Lie algebra. The object of the present paper is to make a deeper study of the representations of the algebra. We shall show that every irreducible representation of the group (at least in case it is complex) is infinitesimally equivalent to one constructed in a certain standard way (Theorem 4). This is the principal result of this paper. Although the proof is rather long the idea behind it is quite simple and has been employed before (see [3]). It can be explained as follows. Let $\mathfrak{B}$ be the universal enveloping algebra of the Lie algebra $g$ of our group. The finitedimensional representations of $\mathfrak{B}$ are very well known and their knowledge enables us to deduce certain algebraic identities in $\mathfrak{B}$. Since these identities must be preserved in every representation, they provide us with useful information about infinite-dimensional representations as well. Roughly speaking one can say that the representations of finite degree are characterised by a certain set of parameters which take integral values. If we keep to the same algebraic pattern but give arbitrary complex values to these parameters we obtain the infinite-dimensional representations.

The results of this paper were obtained in the summer of 1951 and they have been announced in a short note [4(b)].

1. Proof of Theorem 1. Let $g_{0}$ be a semisimple Lie algebra over the field $R$ of real numbers. We denote by $\mathfrak{g}$ its complexification. Define $\mathfrak{l}, \mathfrak{p}, \mathfrak{t}_{0}$, and $\mathfrak{p}_{0}$ as in [5, Part I]. Let $\mathrm{c}$ be the center of $\mathfrak{t}$ and $\mathfrak{t}^{\prime}$ the derived algebra of $\mathfrak{t}$. Then $\mathfrak{l}^{\prime}$ is semisimple and $\mathfrak{t}$ is the direct sum of $\mathfrak{c}$ and $\mathfrak{t}^{\prime}$. Moreover $\mathfrak{t}$ is reductive in $\mathfrak{g}$ (see [5, Lemma 3]). Let $\Omega^{\prime}$ be the set of all equivalence classes of finitedimensional simple representations of $\mathfrak{t}^{\prime}$. Let $\Omega_{F}^{\prime}$ denote the subset consisting of all $\mathfrak{D}^{\prime} \in \Omega^{\prime}$ which occur in the reduction of some finite-dimensional representation of $\mathfrak{g}$ with respect to $\mathfrak{l}^{\prime}$. Let $\mathfrak{B}$ be the universal enveloping algebra of $\mathfrak{g}$ and $\mathfrak{X}, \mathfrak{X}^{\prime}$ the subalgebras of $\mathfrak{B}$ generated by $(1, \mathfrak{t})$ and $\left(1, \mathfrak{t}^{\prime}\right)$ respectively. Our main object at present is to prove the following theorem which provides the basis for most of our subsequent arguments.

THEOREM 1. Let $\mathfrak{Y}^{\prime}$ be a maximal left ideal in $\mathfrak{X}^{\prime}$ such that the natural representation $\left({ }^{1}\right)$ of $\mathfrak{l}^{\prime}$ on $\mathfrak{X}^{\prime} / \mathfrak{Y}^{\prime}$ lies in some class in $\Omega_{F^{\prime}}$. Let $F\left(\mathfrak{Y}^{\prime}\right)$ be the set of all maximal left ideals $\mathfrak{M}$ in $\mathfrak{B}$ with the following two properties: (a) $\mathfrak{M} \supset \mathfrak{Y}^{\prime}$; (b) the space $\mathfrak{B} / \mathfrak{M}$ is of finite dimension. Then $\mathfrak{B} \mathfrak{Y}^{\prime}=\bigcap_{\mathfrak{M} \in F\left(\mathfrak{D}^{\prime}\right)} \mathfrak{M}$.

Received by the editors October 30, 1952.

(1) See [5], beginning of $\$ 3$. 
We first introduce some terminology. Let $C$ be the field of complex numbers and $V$ a vector space over $C$. Let $y_{1}, \cdots, y_{r}$ be a finite set of independent commutative indeterminates and $C[y]$ the ring of polynomials in $(y)$ with coefficient in $C$. Consider the tensor product $V \times C[y]$ which we regard as a twosided $C[y]$-module so that if $f, g \in C[y]$ and $v \in V$ we have $f(v \times g)=(v \times g) f$ $=v \times f g$. We may identify $V$ with a subspace of this tensor product under the mapping $v \rightarrow v \times 1$. Then every element in $V \times C[y]$ can be written as a sum of elements of the form $v f$ where $v \in V$ and $f \in C[y]$. We shall often refer to the elements of $V \times C[y]$ as polynomials in $(y)$ with coefficients in $V$. In accordance with this interpretation we shall write $V[y]$ to denote $V \times C[y]$. The value of such a polynomial at the point $y_{j}=\mu_{j}, 1 \leqq j \leqq r\left(\mu_{j} \in C\right)$, is the element in $V$ obtained by replacing each $y_{j}$ by $\mu_{j}$. If $V$ is an algebra we define multiplication in $V[y]$ according to the rule $\left(v_{1} f_{1}\right)\left(v_{2} f_{2}\right)=\left(v_{1} v_{2}\right)\left(f_{1} f_{2}\right)\left(v_{1}, v_{2} \in V ; f_{1}, f_{2}\right.$ $\in C[y])$. This makes $V[y]$ into an algebra.

Now consider the symmetric algebra $S(\mathfrak{g})$ over $\mathfrak{g}$. We identify $S(\mathfrak{g})$ with the algebra of polynomial functions on $\mathfrak{g}$ as explained in [5]. Moreover if $\mathfrak{l}$ is a linear subspace of $\mathfrak{g}$ the symmetric algebra $S(\mathfrak{l})$ may be regarded as a subalgebra of $S(\mathfrak{g})$. Let $\lambda$ denote the canonical mapping of $S(\mathfrak{g})$ into $\mathfrak{B}$ which was defined in $[5, \S 2]$. Since $\mathfrak{g}$ is the direct sum of $\mathfrak{p}, \mathfrak{l}^{\prime}$, and $\mathfrak{c}$, it follows from Lemma 10 of [5] that the mapping $(f, g, h) \rightarrow \lambda(f) \lambda(g) \lambda(h)$ is a linear isomorphism of the tensor product $S(\mathfrak{p}) \times S\left(\mathfrak{l}^{\prime}\right) \times S(\mathfrak{c})$ with $\mathfrak{B}$. Put $\mathfrak{B}=\lambda(S(\mathfrak{p}))$, $\mathfrak{E}=\lambda(S(\mathfrak{c}))$. If $\left(w_{i}\right)_{i \in I},\left(x_{j}\right)_{j \in I^{\prime}},\left(\gamma_{k}\right)_{k \in I^{\prime \prime}}$ are bases for $\mathfrak{B}, \mathfrak{X}^{\prime}$, and $\mathfrak{E}$ respectively, then $w_{i} x_{j} \gamma_{k}$ and $x_{j} \gamma_{k}$ respectively are bases for $\mathfrak{B}$ and $\mathfrak{X}$. This fact should be constantly borne in mind during the following discussion.

We know that the simply-connected analytic group $G$ corresponding to $\mathfrak{g}_{k}=\mathfrak{f}_{0}+(-1)^{1 / 2} \mathfrak{p}_{0}$ is compact. Let $K$ and $K^{\prime}$ be the analytic subgroups of $G$ corresponding to $\mathfrak{l}_{0}$ and $\mathfrak{t}_{0}^{\prime}=\mathfrak{t}^{\prime} \cap \mathfrak{t}_{0}$. We can choose a base $\Gamma_{1}^{\prime}, \cdots, \Gamma_{r}^{\prime}$ for $\mathfrak{c}_{0}=\mathfrak{c} \cap \mathfrak{f}_{0}$ over $R$ such that $\exp \left(t_{1} \Gamma_{1}^{\prime}+\cdots+t_{r} \Gamma_{r}^{\prime}\right) \in K^{\prime}\left(t_{1}, \cdots, t_{r} \in R\right)$ if and only if $t_{j} / 2 \pi, 1 \leqq j \leqq r$, are all integers. Put $\Gamma_{j}=(-1)^{1 / 2} \Gamma_{j}^{\prime}$. Then $\Gamma_{1}, \cdots$, $\Gamma_{r}$ is a base for $\mathrm{c}$ over $C$. Let $M$ be a linear function on $\mathrm{c}$. We shall say that $M$ is integral if $m_{j}=M\left(\Gamma_{j}\right)$ are all integers $\geqq 0$ and in that case we write $\Gamma^{M}$ $=\Gamma_{1}^{m_{1}} \Gamma_{2}^{m_{2}} \cdots \Gamma_{r}^{m_{r}}$. It is clear that every element $b \in \mathfrak{B}$ can be written uniquely in the form $b=\sum w_{M} \Gamma^{M}$ where $w_{M} \in \mathfrak{P X ^ { \prime }}$ and $M$ runs over all integral functions. Let $y_{1}, \cdots, y_{r}$ be $r$ independent commuting indeterminates. Put $y^{M}=y_{1}^{m_{1}} \cdots y_{r}^{m_{r}}$ where $m_{j}=M\left(\Gamma_{j}\right)$ and $M$ is integral. Then if we put $\alpha_{y}(b)$ $=\sum_{M} w_{M} y^{M}$, the mapping $\alpha_{y}$ is a linear isomorphism of $\mathfrak{B}$ with $\mathfrak{B X ^ { \prime }}[y]$. If $\mu$ is a linear function on $\mathrm{c}$ we shall denote by $\alpha_{\mu}(b)$ the value of the polynomial $\alpha_{y}(b)$ at the point $y_{j}=\mu\left(\Gamma_{j}\right), 1 \leqq j \leqq r$.

Now we come to the proof of Theorem 1 which would consist of three stages.

First reduction. Let $b_{0}$ be an element in $\mathfrak{B}$ which is not in $\mathfrak{B} \mathfrak{Y}^{\prime}$. Then we have to find an $\mathfrak{M} \in F\left(\mathfrak{Y}^{\prime}\right)$ such that $b_{0} \notin \mathfrak{M}$. We can write $b_{0}$ in the form $b_{0}=\sum_{M} w_{M} \Gamma^{M}\left(w_{M} \in \mathfrak{B X} \mathfrak{X}^{\prime}\right)$. Since $b_{0} \notin \mathfrak{B \mathfrak { Y } ^ { \prime }}$, the elements $w_{M}$ cannot all lie 
in $\mathfrak{B Y} \mathfrak{Y}^{\prime}$. Hence $\alpha_{y}\left(b_{0}\right)=\sum_{M} w_{M} y^{M} \notin \mathfrak{P Y} \mathfrak{Y}^{\prime}[y]$. Let $w_{1}, \cdots, w_{j}$ be a maximal set of elements among $w_{M}$ which are linearly independent mod $\mathfrak{B Y} \mathfrak{Y}^{\prime}$. Then $\left.\alpha_{y}\left(b_{0}\right) \equiv \sum_{i=1}^{s} w_{i} f_{i}(y) \bmod \mathfrak{B Y}\right)^{\prime}[y]$ where $f_{i}(y) \in C[y]$ and not all $f_{i}(y)$ are zero. So let us assume $f_{1}(y) \neq 0$. Then we can find an integral linear function $\mu$ on $c$ such that $f_{1}(\mu) \neq 0\left(f_{1}(\mu)\right.$ is the value of the polynomial $f_{1}(y)$ at $\dot{y}_{j}=\mu\left(\Gamma_{j}\right)$, $1 \leqq j \leqq r)$. Put $\mathfrak{Y}=\mathfrak{X Y} \mathfrak{Y}^{\prime}+\sum_{j=1}^{r} \mathfrak{X}\left(\Gamma_{j}-\mu\left(\Gamma_{j}\right)\right)$. Then $\mathfrak{Y}$ is a left ideal in $\mathfrak{X}$. It would be sufficient to find a maximal left ideal $\mathfrak{M}$ in $\mathscr{B}$ such that $b_{0} \in \mathfrak{M}$, $\mathfrak{M} \supset \mathfrak{Y}$, and $\operatorname{dim} \mathfrak{B} / \mathfrak{M}<\infty$. First of all we claim that $b_{0} \in \mathfrak{B} \mathfrak{Y}$. For since $\Gamma_{j} \equiv \mu\left(\Gamma_{j}\right) \bmod \mathfrak{Y}(1 \leqq j \leqq r), b_{0} \equiv \alpha_{\mu}\left(b_{0}\right) \bmod \mathfrak{B Y}$. Therefore if $b_{0} \in \mathfrak{B} \mathfrak{Y}, \alpha_{\mu}\left(b_{0}\right)$ $\in \mathfrak{B Y} \cap \mathfrak{P} \mathfrak{X}^{\prime}$. But $\mathfrak{B Y}=\mathfrak{B Y}$ and therefore $\mathfrak{B Y} \cap \mathfrak{B} \mathfrak{X}^{\prime}=\mathfrak{B}\left(\mathfrak{Y} \cap \mathfrak{X}^{\prime}\right)=\mathfrak{P Y} \mathfrak{Y}^{\prime}$. (In order to prove these statements we have to make use of our earlier remarks about bases.) Hence $\alpha_{\mu}\left(b_{0}\right) \in \mathfrak{P Y}^{\prime}$. But $\left.\alpha_{\mu}\left(b_{0}\right) \equiv \sum_{i=1}^{s} f_{i}(\mu) w_{i} \bmod \mathfrak{P Y}\right)^{\prime}$ and $w_{i}(1 \leqq i \leqq s)$ are linearly independent $\bmod \mathfrak{B Y ^ { \prime }}$ and $f_{1}(\mu) \neq 0$. Therefore $\alpha_{\mu}\left(b_{0}\right) \notin \mathfrak{B Y} \mathfrak{Y}^{\prime}$ and we get a contradiction. This shows that $b_{0} \notin \mathfrak{B} \mathfrak{Y}$. Moreover since $\mathfrak{Y}^{\prime}=\mathfrak{X}^{\prime} \cap \mathfrak{Y}$ and $\Gamma \equiv \mu(\Gamma) \bmod \mathfrak{Y}(\Gamma \in \mathfrak{c})$ we may identify $\mathfrak{X} / \mathfrak{Y}$ and $\mathfrak{X}^{\prime} / \mathfrak{Y}^{\prime}$ in a natural way. Let $\rho$ be the natural representation of $\mathfrak{l}$ on $\mathfrak{X} / \mathfrak{Y}$. Then $\rho$ coincides on $\mathfrak{l}^{\prime}$ with its natural representation $\rho^{\prime}$ on $\mathfrak{X}^{\prime} / \mathfrak{Y}^{\prime}$. Since the equivalence class of $\rho^{\prime}$ lies in $\Omega_{F}^{\prime}$ it is clear that $\rho^{\prime}$ can be extended to a representation of $K^{\prime}$ on $\mathfrak{X}^{\prime} / \mathfrak{Y}^{\prime}$. Hence if we bear in mind the fact that the linear function $\mu$ is integral, we see easily that $\rho$ can also be extended to a representation of $K$ on $\mathfrak{X} / \mathfrak{Y}$. Now $K$ is a closed $\left({ }^{2}\right)$ subgroup of the compact group $G$ and therefore every finite-dimensional simple representation of $K$ occurs in the reduction of some representation of $G$ of the same type. This shows that the class $\mathfrak{D}$ of $\rho$ occurs in the reduction of some finite-dimensional representation of $\mathrm{g}$. Hence it is sufficient to prove the following lemma.

LEммA 1. Let $\mathfrak{Y}$ be a maximal left ideal in $\mathfrak{X}$ such that the natural representation of $\mathfrak{l}$ on $\mathfrak{X} / \mathfrak{Y}$ is finite-dimensional and its equivalence class occurs in the reduction of some finite-dimensional representation of $\mathfrak{g}$ with respect to $\mathfrak{t}$. Then

$$
\mathfrak{B Y}=\bigcap_{\mathfrak{M} \in F(\mathfrak{D})} \mathfrak{M}
$$

where $F(\mathfrak{Y})$ is the set of all left ideals $\mathfrak{M}$ in $\mathfrak{B}$ containing $\mathfrak{Y}$ for which $\operatorname{dim} \mathfrak{B} / \mathfrak{M}$ $<\infty$.

The second step. We shall first prove this lemma in the special case when $\mathfrak{V}=\mathfrak{X f}$. Let $\Sigma$ denote the set of all finite-dimensional simple representations $\pi$ of $\mathfrak{g}$ such that the zero representation of $\mathfrak{f}$ occurs in $\pi$. Let $\mathfrak{n}_{\boldsymbol{\pi}}$ denote the kernel of $\pi$ in $\mathfrak{g}$.

(2) This is seen as follows. We know that $\mathfrak{t}_{0}$ is the subalgebra of $g_{k}$ which consists of all points which are left fixed by an automorphism of $\mathfrak{g}_{k}$ of order two. $K$ is therefore the component of identity of the subgroup of $G$ consisting of the fixed points of the corresponding automorphism of $G$. Hence $K$ is closed. 


\section{Lemma 2. $\bigcap_{\pi \in \Sigma} \mathfrak{n}_{\boldsymbol{x}} \subset \mathfrak{l}$.}

Consider the compact groups $G$ and $K$ defined above and let $V$ be the factor space $G / K$ consisting of all cosets of the form $x K(x \in G)$. Then $G$ operates on $V$ in the usual fashion. Let $C(V)$ be the space of all continuous functions on $V$. For any $f \in C(V)$ and $x \in G$ define $\rho(x) f$ as the function $v \rightarrow f\left(x^{-1} v\right)(v \in V)$ on $V$. Then the mapping $\rho: x \rightarrow \rho(x)$ is a representation of $G$ on $C(V)$. Let $C_{0}(V)$ be the set of those elements $f \in C(V)$ whose transforms $\rho(x) f(x \in G)$ span a finite-dimensional subspace. Then it is well known that every continuous function on $V$ is the uniform limit of functions in $C_{0}(V)$. Let us denote by $v_{0}$ the coset $K$ regarded as a point of $V$. Then if $x \notin K, x^{-1} v_{0} \neq v_{0}$. Hence we can find a continuous function $f$ on $V$ such that $f\left(x^{-1} v_{0}\right) \neq f\left(v_{0}\right)$. Since $f$ can be approximated uniformly by elements in $C_{0}(V)$ we may assume that $f$ lies in $C_{0}(V)$. Let $U$ be the linear space spanned by $\rho(y) f(y \in G)$ and let $\rho_{U}$ be the representation of $G$ induced on $U$. Then $\rho_{U}$ is a finite-dimensional continuous representation of $G$ and since $\rho(x) f \neq f, x$ does not lie in the kernel of $\rho_{U} . G$ being compact, $\rho_{U}$ is fully reducible and so we can find a simple component $\sigma_{x}$ of $\rho_{U}$ such that $x$ is not in the kernel of $\sigma_{x}$. On the other hand it follows from the Frobenius reciprocity relation (see Weil $[7$, p. 83]) that the trivial representation of $K$ occurs in $\sigma_{x}$. Now let $X \in \mathfrak{g}_{k}, X \notin \mathfrak{f}_{0}$. Then we can find a real number $t$ such that $\exp t X \notin K$. Consider the representation $\sigma_{x}$ corresponding to $x=\exp t X$. Then if $\pi$ is the representation of $\mathrm{g}_{k}$ (and therefore of $\mathfrak{g}$ ) corresponding to $\sigma_{x}$ it is clear that $\pi \in \Sigma$ and $X \notin \mathfrak{n}_{\mathfrak{x}}$. This shows $\mathfrak{n} \cap \mathfrak{g}_{k}$ $\subset \mathfrak{l}_{0}$ where $\mathfrak{n}=\bigcap_{\pi \in \Sigma} \mathfrak{n}_{\boldsymbol{\pi}}$. Let $\eta$ be the conjugation of $\mathfrak{g}$ with respect to $\mathfrak{g}_{k}$ so that $\eta\left(X+(-1)^{1 / 2} Y\right)=X-(-1)^{1 / 2} Y\left(X, Y \in \mathfrak{g}_{k}\right)$. Let $\Sigma_{0}$ be the set of all matrix representations in $\Sigma$. Then it is clear that $\mathfrak{n}=\bigcap_{\pi \in \Sigma_{0}} \mathfrak{n}_{\pi}$. Now corresponding to any $\pi \in \Sigma_{0}$ define a representation $\bar{\pi}$ by the rule $\bar{\pi}(X)=\overline{\pi(\eta(X))}$ $(X \in g)$ where $\overline{\pi(\eta(X))}$ is the complex-conjugate of the matrix $\pi(\eta(X))$. It is obvious that $\bar{\pi} \in \Sigma_{0}$, and $\mathfrak{n}_{\bar{\pi}}=\eta\left(\mathfrak{n}_{\mathfrak{r}}\right)$ and therefore $\eta(\mathfrak{n})=\mathfrak{n}$. Hence if $X \in \mathfrak{n}$, $X+\eta(X)$ and $(-1)^{1 / 2}(X-\eta(X))$ are both in $\mathfrak{n} \cap \mathfrak{g}_{k} \subset \mathfrak{f}_{0}$. Therefore $X \in \mathfrak{l}$ and this proves the lemma.

Now let $\mathfrak{A}$ be the intersection of all left ideals in $F(\mathfrak{X} \mathfrak{f})$. We have to prove that $\mathfrak{A}=\mathfrak{B} \mathfrak{f}$. First we shall show that $\mathfrak{A} \cap \mathfrak{g}=\mathfrak{l}$. Since $\mathfrak{A} \supset \mathfrak{f}$ it is enough to prove that $\mathfrak{A} \cap \mathfrak{p}=\{0\}$. In order to do this we make use of an argument due to E. Cartan [1]. Let $\mathfrak{p}_{1}=\mathfrak{A} \cap \mathfrak{p}$ and let $\mathfrak{p}_{2}$ be the set of all $Y \in \mathfrak{p}$ such that $B(X, Y)$ $=\operatorname{sp}(\operatorname{ad} X$ ad $Y)=0$ for all $X \in \mathfrak{p}_{1}$. Since the bilinear form $B$ is nondegenerate on $\mathfrak{g}$ and since $\mathfrak{f}$ and $\mathfrak{p}$ are orthogonal under $B$ it follows that $B$ is nondegenerate on $\mathfrak{p}$ and therefore $\mathfrak{p}=\mathfrak{p}_{1}+\mathfrak{p}_{2}, \mathfrak{p}_{1} \cap \mathfrak{p}_{2}=\{0\}$. Let $X \in \mathfrak{p}_{1}, Y \in \mathfrak{p}_{2}$, and $Z \in \mathfrak{l}$. Then $B([X, Y], Z)=-B(Y,[X, Z])$. Since $\mathscr{A}$ is a left ideal containing $\mathfrak{l}$ and $[\mathfrak{l}, \mathfrak{p}] \subset \mathfrak{p}$ it is clear that $\left[\mathfrak{t}, \mathfrak{p}_{1}\right] \subset \mathfrak{p}_{1}$. Hence $[X, Z] \in \mathfrak{p}_{1}$ and therofore $B(Y,[X, Z])=0$. This means that $B([X, Y], Z)=0$ for all $Z \in f$. On the other hand $[X, Y] \in[\mathfrak{p}, \mathfrak{p}] \subset \mathfrak{l}$ and since $B$ is nondegenerate on $\mathfrak{f}$ it follows that $[X, Y]=0$. Hence $\left[p_{1}, p_{2}\right]=\{0\}$. Moreover $\left[\mathfrak{f}, \mathfrak{p}_{1}\right] \subset \mathfrak{p}_{1}$ and therefore 
$\left[\mathfrak{l}, \mathfrak{p}_{2}\right] \subset \mathfrak{p}_{2}$. Let $\mathfrak{l}$ be the centraliser of $\mathfrak{p}_{2}$ in $\mathfrak{l}$. Then we claim that $\mathfrak{p}_{1}+\mathfrak{l}$ is an ideal in $g$. Since $\left[p_{1}+\mathfrak{l}, p_{2}\right]=\{0\}$ it follows that

$$
\left[\mathfrak{p}_{1}+\mathfrak{l}, \mathfrak{p}\right]=\left[\mathfrak{p}_{1}+\mathfrak{l}, \mathfrak{p}_{1}\right]=\left[\mathfrak{p}_{1}, \mathfrak{p}_{1}\right]+\left[\mathfrak{l}, \mathfrak{p}_{1}\right] .
$$

But $\left[p_{1}, p_{1}\right] \subset \mathfrak{l}$ and $\left[p_{2},\left[p_{1}, p_{1}\right]\right]=\{0\}$. Hence $\left[p_{1}, p_{1}\right] \subset \mathfrak{l}$. Furthermore we know that $\left[\mathfrak{f}, \mathfrak{p}_{1}\right] \subset \mathfrak{p}_{1}$. Therefore

$$
\left[\mathfrak{p}_{1}+\mathfrak{l}, \mathfrak{p}\right] \subset \mathfrak{l}+\mathfrak{p}_{1} .
$$

On the other hand since $\left[\mathfrak{l}, \mathfrak{p}_{2}\right] \subset \mathfrak{p}_{2}$ it is obvious that $[\mathfrak{l}, \mathfrak{l}] \subset \mathfrak{l}$. Hence $\left[\mathfrak{p}_{1}+\mathfrak{l}, \mathfrak{l}\right] \subset \mathfrak{p}_{1}+\mathfrak{l}$. This proves that $\mathfrak{p}_{1}+\mathfrak{l}$ is an ideal in $\mathfrak{g}$ and it is clear that $\mathfrak{A} \supset \mathfrak{p}_{1}+\mathfrak{l}$.

Now suppose $p_{1} \neq\{0\}$ and let $X \neq 0$ be an element in $p_{1}$. Then from Lemma 2 we can find a finite-dimensional simple representation $\pi$ of $g$ such that $\pi(X) \neq 0$ and the zero representation of $t$ occurs in $\pi$. Choose a vector $\psi \neq 0$ in the representation space such that $\pi(Z) \psi=0$ for all $Z \in \mathfrak{l}$. We extend $\pi$ to a representation of $\mathscr{B}$ and consider the set. $\mathfrak{M}$ of all elements $b \in \mathfrak{B}$ such that $\pi(b) \psi=0$. Then it is obvious that $\mathfrak{M} \in F(\mathfrak{X} \mathfrak{i})$ and therefore $\mathfrak{M} \supset \mathfrak{A} \supset \mathfrak{p}_{1}+\mathfrak{l}$. Since $\pi$ is irreducible and $\mathfrak{p}_{1}+\mathfrak{l}$ is an ideal in $\mathfrak{g}$ and since $\pi\left(\mathfrak{p}_{1}+\mathfrak{l}\right) \psi \subset \pi(\mathfrak{M}) \psi$ $=\{0\}$, it follows that $\pi(Y)=0$ for all $Y \in \mathfrak{p}_{1}+\mathfrak{l}$. Therefore in particular $\pi(X)$ $=0$. Since this contradicts our choice of $\pi, p_{1}=\{0\}$.

Let $\mathfrak{p}^{\prime}$ be the linear subspace of $\mathfrak{B}$ spanned by the set $(\mathfrak{p}, 1)$. We claim that $\mathfrak{p}^{\prime} \cap \mathfrak{A}=\{0\}$. For suppose $c+Y \in \mathfrak{A}(c \in C, Y \in \mathfrak{p})$. Then it follows from the above result that if $Y \neq 0$ we can find an irreducible representation $\pi$ of $\mathfrak{g}$ on a finite-dimensional space $V$ and a vector $\psi \neq 0$ in $V$ such that $\pi(X) \psi=0$ for all $X \in \mathfrak{l}$ and $\pi(Y) \psi \neq 0$. Consider the representation $\pi_{2}$ of $\mathfrak{g}$ (and therefore of $\mathfrak{B}$ ) induced on $V \times V$. Let $\mathfrak{M}_{\pi}$ and $\mathfrak{M}_{\pi_{2}}$ respectively be the set of those elements $b \in \mathfrak{B}$ for which $\pi(b) \psi=0$ and $\pi_{2}(b)(\psi \times \mathbf{X} \psi)=0$. Then it is obvious that $\mathfrak{M}_{\pi}$ and $\mathfrak{M}_{\pi_{2}}$ are both in $F(\mathfrak{X} \mathfrak{l})$ and therefore $\mathfrak{A} \subset \mathfrak{M}_{\pi} \cap \mathfrak{M}_{x_{2}}$. Now let $Y^{\prime}=c+Y$. Then

$$
\begin{aligned}
0 & =\pi_{2}\left(Y^{\prime}\right)(\psi \times \psi \psi)=\pi\left(Y^{\prime}\right) \psi \times \psi \psi+\psi \times \pi\left(Y^{\prime}\right) \psi-c(\psi \times \\
& =-c(\psi \times \psi \psi
\end{aligned}
$$

since $Y^{\prime} \in \mathfrak{A}$. Hence $c=0$ and therefore $Y \in \mathfrak{A} \cap \mathfrak{p}=\{0\}$. This proves that $\mathscr{A} \cap \mathfrak{p}^{\prime}=\{0\}$. Since $\operatorname{dim} \mathfrak{p}^{\prime}$ is finite it follows that we can find a finite set $\mathfrak{M}_{1}, \cdots, \mathfrak{M}_{8}$ of left ideals in $F(\mathfrak{X} \mathfrak{i})$ such that $\mathfrak{M}_{1} \cap \ldots \cap \mathfrak{M}_{8} \cap \mathfrak{p}^{\prime}=\{0\}$. Put $\mathfrak{M}_{0}=\mathfrak{M}_{1} \cap \ldots \cap \mathfrak{M}_{\mathfrak{s}}$. Then $\mathfrak{M}_{0} \supset \mathfrak{X l}$ and $\operatorname{dim} \mathfrak{B} / \mathfrak{M}_{0} \leqq \sum_{i=1}^{s} \operatorname{dim} \mathfrak{B} / \mathfrak{M}_{i}$ $<\infty$. Hence $\mathfrak{M}_{0} \in F(\mathfrak{X} \mathfrak{i})$.

We are now in a position to prove Lemma 1 in the case $\mathfrak{V}=\mathfrak{X}$. Choose a base $Y_{1}, \cdots, Y_{p}$ for $p$ and let $Q$ be any monomial in $S(\mathfrak{p})$ constructed from the elements of this base. Then the elements $\lambda(Q)$ taken together for all $Q$ form a base for $\mathfrak{B}$. Since $\mathfrak{B}=\mathfrak{B X}$ it follows that $\mathfrak{B}=\mathfrak{B}+\mathfrak{B} \mathfrak{P}$. Now let $b_{0}$ be an element of $\mathfrak{B}$ which is not in $\mathfrak{B} \mathfrak{l}$. Then $b_{0} \equiv w \bmod \mathfrak{B} \mathfrak{l}$ where $w \in \mathfrak{P}$ and $w \neq 0$. Since $w$ is a linear combination of $\lambda(Q)$ it could be written in the form 


$$
w=\sum_{0 \leqq r \leqq M} \sum_{1 \leqq i_{1}, \cdots, i_{r} \leqq p} a^{i_{1} i_{2} \cdots i_{r}} Y_{i_{1}} Y_{i_{2}} \cdots Y_{i_{r}} \quad\left(a^{i_{1} i_{2}} \cdots i_{r} \in C\right)
$$

where the $a$ 's are symmetric, i.e. $a^{i_{1} i_{2} \cdots i_{r}}=a^{i_{j_{1}} i_{2} \cdots i_{i_{r}}}$ for any permutation $\left(j_{1}, \cdots, j_{r}\right)$ of $(1,2, \cdots, r)$. We choose $M$ in such a way that some $a^{i_{1} i_{2} \cdots i_{M}} \neq 0$. Let $b \rightarrow b^{*}$ denote the natural mapping and $\pi$ the natural representation of $\mathfrak{B}$ on $\mathfrak{B} / \mathfrak{M}_{0}=\mathfrak{B}^{*}$. We know that $\mathfrak{M}_{0} \supset \mathfrak{l}$ and $\operatorname{dim} \mathfrak{B}^{*}<\infty$. Let $\mathfrak{B}_{\boldsymbol{M}}^{*}$ denote the Kronecker product of $\mathfrak{B}^{*}$ with itself $M$ times. Since $\mathfrak{M}_{0} \cap \mathfrak{p}^{\prime}=\{0\}$ it follows that $1^{*}, Y_{1}^{*}, \cdots, Y_{p}^{*}$ are linearly independent in $\mathfrak{B}^{*}$. Choose a base $z_{j}^{*}, 0 \leqq j \leqq h$, for $\mathfrak{B}^{*}$ such that $z_{0}^{*}=1^{*}$ and $z_{i}^{*}=Y_{i}^{*}, 1 \leqq i \leqq p$, and let $E_{M}^{*}$ be the subspace of $\mathfrak{B}_{M}^{*}$ spanned by elements of the form $z_{t_{1}}^{*} \times z_{t_{2}}^{*} \times \ldots$ $\mathrm{X} z_{i_{M}}^{*}\left(0 \leqq i_{1}, \cdots, i_{M} \leqq h\right)$ where $i_{r}=0$ for at least one $r(1 \leqq r \leqq M)$. Let $\pi_{M}$ denote the representation of $\mathfrak{g}$ (and therefore of $\mathfrak{B}$ ) induced on the Kronecker product $\mathfrak{B}_{M}^{*}$. Then it is easy to verify (see $[2$, p. 904]) that

$\pi_{M}(w)\left(1^{*} \times 1^{*} \times \cdots \times 1^{*}\right)$

$$
\equiv M ! \sum_{1 \leqq i_{1}, \cdots, i_{M} \leqq p} a^{i_{1} i_{2} \cdots i_{M}}\left(Y_{i_{1}}^{*} \times \cdots \times Y_{i_{M}}^{*}\right) \bmod E_{M}^{*} .
$$

Since $z_{j_{1}}^{*} \times z_{j_{2}}^{*} \times \cdots \times z_{j_{M}}^{*}\left(0 \leqq j_{1}, \cdots, j_{M} \leqq h\right)$ is a base for $\mathfrak{B}_{M}^{*}$, the elements $Y_{i_{1}}^{*} \times Y_{i_{2}}^{*} \times \cdots \times Y_{i_{M}}^{*}\left(1 \leqq i_{1}, \cdots, i_{M} \leqq p\right)$ are linearly independent $\bmod E_{M}^{*}$.

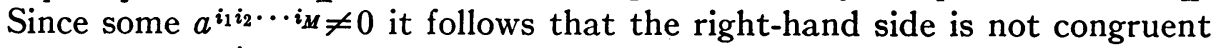
to zero $\bmod E_{M}^{*}$ and therefore $\pi_{M}(w)\left(1^{*} \times 1^{*} \times \cdots \times 1^{*}\right) \neq 0$. On the other hand since $\mathfrak{M}_{0} \supset$ it is clear that $\pi_{M}(X)\left(1^{*} \times 1^{*} \times \cdots \times 1^{*}\right)=0$ for all $X \in \mathfrak{l}$. Therefore $\pi_{M}\left(b_{0}\right)\left(1^{*} \times 1^{*} \times \cdots \times 1^{*}\right)=\pi_{M}(w)\left(1^{*} \times \cdots \times 1^{*}\right) \neq 0$. Let $\mathfrak{M}_{M}$ be the set of all elements $b \in \mathfrak{B}$ such that $\pi_{M}(b)\left(1^{*} \times \cdots \times 1^{*}\right)=0$. Then $b_{0} \in \mathfrak{M}_{M}$ and $\mathfrak{M}_{M} \in F(\mathfrak{X} \mathfrak{l})$ since $\operatorname{dim} \mathfrak{B} / \mathfrak{M}_{M} \leqq \operatorname{dim} \mathfrak{B}_{M}^{*}<\infty$. This shows that $b_{0} \in \mathfrak{A}$ and therefore we conclude that $\mathfrak{A}=\mathfrak{B} \mathfrak{l}$.

The final step. Now we come to the general case of Lemma 1. We recall that $\mathfrak{P}$ is the image of $S(\mathfrak{p})$ under $\lambda$. First we prove the following lemma.

Lemma 3. Let $w_{1}, \cdots, w_{r}$ be a finite set of elements in $\mathfrak{P}$ which are linearly independent. Then there exists a left ideal $\mathfrak{M} \in F(\mathfrak{X})$ such that $w_{1}, \cdots, w_{r}$ are linearly independent modulo $\mathfrak{M}$.

Let $W$ be the space spanned by $w_{1}, \cdots, w_{r}$. Then $W \cap \mathfrak{B l} \subset \mathfrak{B} \cap \mathfrak{B l}$ $=\mathfrak{B} \cap \mathfrak{B X f}$. Since $\lambda$ is a linear isomorphism of $S(\mathfrak{p}) \times S(\mathfrak{l})$ onto $\mathfrak{B}$ it follows that $\mathfrak{B} \cap \mathfrak{B X f}=\{0\}$ and therefore $W \cap \mathfrak{B P}=\{0\}$. Since $\operatorname{dim} W<\infty$ it follows from what we have proved above that we can find a finite set of elements $\mathfrak{M}_{1}, \cdots, \mathfrak{M}_{8}$ in $F(\mathfrak{X} \mathfrak{i})$ such that $W \cap \mathfrak{M}_{1} \cap \cdots \cap \mathfrak{M}_{8}=\{0\}$. Put $\mathfrak{M}=\mathfrak{M}_{1}$ $\cap \cdots \cap \mathfrak{M}_{s}$. Then $\mathfrak{M} \supset \mathfrak{l}$ and $\operatorname{dim} \mathfrak{B} / \mathfrak{M} \leqq \sum_{1 \leqq i \leqq o} \operatorname{dim} \mathfrak{B} / \mathfrak{M}_{i}<\infty$ and therefore $\mathfrak{M} \in F(\mathfrak{X} \mathfrak{f})$. Since $\mathfrak{M} \cap W=\{0\}$ our assertion is proved.

Let $\mathfrak{D}_{0}$ be the (equivalence) class of the natural representation of $\mathfrak{t}$ on $\mathfrak{X} / \mathfrak{Y}$ in Lemma 1 . Then we can find an irreducible representation $\sigma$ of $\mathfrak{g}$ (and therefore of $\mathfrak{B}$ ) on a finite-dimensional space $V$ and a vector $\psi \neq 0$ in $V$ such 
that $\sigma(\mathfrak{Y}) \psi=\{0\}$. Let $\mathfrak{B}_{1}$ be the set of all elements $b \in \mathfrak{B}$ such that $\sigma(b) \psi=0$. Then $\mathfrak{B}_{1} \supset \mathfrak{Y}$ and we may regard $\sigma$ as the natural representation of $\mathfrak{B}$ on $\mathfrak{B} / \mathfrak{B}_{1}$.

Let $a$ be an element in $\mathfrak{B}$ which is not in $\mathfrak{B V}=\mathfrak{B V}$. Since $\lambda(Q)$ form a base for $\mathfrak{B}$ we can write $a$ in the form $a=\sum_{Q} \lambda(Q) x_{Q}$ where $x_{Q} \in \mathfrak{X}$ and not all $x_{Q}$ are in $\mathfrak{Y}$. Let $|Q|$ denote the degree of the monomial $Q$ and let $M$ be the highest integer for which there exists a monomial $Q_{0}$ with degree $M$ such that $x_{Q_{0}} \notin \mathfrak{Y}$. From Lemma 3 there exists a left ideal $\mathfrak{B} \in F(\mathfrak{X} \mathfrak{)})$ such that the elements $\lambda(Q)(|Q| \leqq M)$ are linearly independent $\bmod \mathfrak{B}$. Let $b \rightarrow b^{*}$ denote the natural mapping and $\pi$ the natural representation of $\mathfrak{B}$ on $\mathfrak{B}^{*}=\mathfrak{B} / \mathfrak{B}$. Similarly let $b \rightarrow b$ denote the natural mapping of $\mathfrak{B}$ on $\bar{B}=\mathscr{B} / \mathfrak{B}_{1}$. Consider the representation $\nu$ of $g$ induced on $\mathfrak{B}^{*} \times \overline{\mathfrak{B}}$. Then it is obvious that (see $[5$, Lemma 13])

$$
\nu(a)\left(1^{*} \times \overline{1}\right)=\sum_{|Q|=M}(\lambda(Q))^{*} \times \bar{x}_{Q}+\sum_{|Q|<M}(\lambda(Q))^{*} \times \bar{b}_{Q} \quad\left(b_{Q} \in \mathfrak{B}\right) .
$$

Since $(\lambda(Q))^{*}(|Q| \leqq M)$ are linearly independent and since not all $\bar{x}_{Q}$ $(|Q|=M)$ are zero it follows that $\nu(a)\left(1^{*} \times \overline{1}\right) \neq 0$. Let $\mathfrak{M}$ be the set of all $b \in \mathfrak{B}$ for which $\nu(b)\left(1^{*} \times \overline{1}\right)=0$. Since $\pi(X) 1^{*}=0 \quad(X \in \mathfrak{l})$ it follows that $\mathfrak{M} \supset \mathfrak{Y}$. Moreover $\operatorname{dim} \mathfrak{B} / \mathfrak{M} \leqq \operatorname{dim}\left(\mathfrak{B}^{*} \times \overline{\mathfrak{B}}\right)<\infty$ and therefore $\mathfrak{M} \in F(\mathfrak{Y})$. Since $a \in \mathfrak{M}$, Lemma 1 and therefore Theorem 1 is proved.

2. Proof of Theorem 2. Let $\mathfrak{A}$ be an associative algebra with 1 and let $\mathfrak{M}_{1}, \mathfrak{M}_{2}$ be two left ideals in $\mathfrak{A}$. Let $\pi_{1}, \pi_{2}$ be the natural representations of $\mathfrak{A}$ on $\mathfrak{A} / \mathfrak{M}_{1}$ and $\mathfrak{A} / \mathfrak{M}_{2}$ respectively. We shall say that $\mathfrak{M}_{1}, \mathfrak{M}_{2}$ are equivalent if there exists a linear isomorphism $\alpha$ of $\mathfrak{A} / \mathfrak{M}_{1}$ onto $\mathfrak{A} / \mathfrak{M}_{2}$ such that $\pi_{2}(a) \alpha$ $=\alpha \pi_{1}(a)(a \in \mathfrak{H})$.

Let $\mathfrak{Q}$ be the centraliser of $\mathfrak{t}$ in $\mathscr{B}$ and $\mathbb{Z}$ the center of $\mathfrak{B}$. If $\chi \neq 0$ is a homomorphism of 3 into $C$ we denote by $B_{x}$ the kernel of $\chi$ in 3 . Similarly for any $\mathfrak{D}_{0} \in \Omega$ we denote by $\mathfrak{N}_{\mathbb{D}_{0}}$ the kernel (in $\mathfrak{X}$ ) of any representation of $\mathfrak{X}$ which lies in $\mathfrak{D}_{0}$.

ThEOREM 2. Let $M\left(\mathfrak{D}_{0}, \chi\right)$ be the set of all maximal left ideals in $\mathfrak{B}$ which contain $\mathfrak{N}_{\mathfrak{D}_{0}}+\mathfrak{Z}_{x}$. Then for any $\mathfrak{M} \in M\left(\mathfrak{D}_{0}, \chi\right), \mathfrak{M} \cap(\mathfrak{O X})$ is a maximal left ideal in $\mathfrak{O X}$. Two elements $\mathfrak{M}_{1}, \mathfrak{M}_{2}$ in $M\left(\mathfrak{D}_{0}, \chi\right)$ are equivalent in $\mathfrak{B}$ if and only if $\mathfrak{M}_{1} \cap \mathfrak{Q X}$ and $\mathfrak{M}_{2} \cap \mathfrak{O X}$ are equivalent in $\mathfrak{O X}$.

First we shall prove the following simple lemma.

Lemma 4. Let $\pi$ be a quasi semisimple representation of $\mathfrak{X}$ on a space $V$. Suppose $\mathfrak{D}_{1}, \cdots, \mathfrak{D}_{N}$ are all the distinct classes in $\Omega$ which occur in $\pi$ and let $V_{\mathfrak{D}_{j}}$ be the set of those elements in $V$ which transform under $\pi$ according to $\mathfrak{D}_{j}$. Then there exist elements $e_{1}, \cdots, e_{N}$ in $\mathfrak{X}$ such that $\pi\left(e_{i}\right) \psi=\psi$ or 0 according as $\psi \in V_{\mathfrak{D}_{i}}$ or $\psi \in V_{\mathfrak{D}_{j}}, j \neq i(1 \leqq i \leqq N)$.

Let $\pi_{j}$ be the representation of $\mathfrak{X}$ induced on $V_{\Phi_{j}}(1 \leqq j \leqq N)$ and let $\mathfrak{R}_{j}$ be the kernel of $\pi_{j}$ in $\mathfrak{X}$. Since the class $\mathfrak{D}_{j}$ is irreducible, $\mathfrak{N}_{j}$ is a maximal two-sided ideal in $\mathfrak{X}$ and $\mathfrak{N}_{i} \neq \mathfrak{N}_{j}$ if $i \neq j$. Put $\mathfrak{N}=\bigcap_{j=1}^{N} \mathfrak{N}_{j}$. Then $\mathfrak{N}$ is the kernel 
of $\pi$ and since $\mathfrak{D}_{1}, \cdots, \mathfrak{D}_{N}$ are all the distinct classes which occur in $\pi$ it is clear that $\mathfrak{N}_{1}, \cdots, \mathfrak{N}_{N}$ are the only maximal ideals in $\mathfrak{X}$ which contain $\mathfrak{N}$. It is obvious that the algebra $\mathfrak{X}^{*}=\mathfrak{X} / \mathfrak{N}$ is finite-dimensional and semisimple and $\mathfrak{Y}_{j}^{*}=\mathfrak{N}_{j} / \mathfrak{R}, 1 \leqq j \leqq N$, are all the distinct maximal ideals in $\mathfrak{X}^{*}$. Let $\mathfrak{X}_{j}^{*}$ be the ideal complementary to $\mathfrak{Y}_{j}^{*}$. Then it follows that $\mathfrak{X}_{1}^{*}, \cdots, \mathfrak{X}_{N}^{*}$ is a complete set of distinct minimal ideals in $\mathfrak{X}^{*}$. Now since $\mathfrak{N}$ is the kernel of $\pi$, we may regard $\pi$ as a faithful representation of $\mathfrak{X}^{*}$ on $V$. Since $\mathfrak{Y}_{j}{ }^{*} \mathfrak{X}_{j}^{*}=\{0\}$ it follows that $\pi\left(\mathfrak{N}_{j}\right) \pi\left(\mathfrak{X}_{j}^{*}\right) V=\{0\}$ and therefore $\pi\left(\mathfrak{X}_{j}^{*}\right) V \subset V_{\mathfrak{D}_{j}}$. Moreover $V=\pi\left(\mathfrak{X}^{*}\right) V=\sum_{j=1}^{N} \pi\left(\mathfrak{X}_{j}^{*}\right) V$. Since the sum $\sum_{j} V_{\mathfrak{D}_{j}}$ is direct we conclude that $\pi\left(\mathfrak{X}_{j}^{*}\right) V=V_{\mathfrak{D}_{j}}$. Let $e_{j}^{*}$ denote the unit element of the simple algebra $\mathfrak{X}_{j}^{*}$. Then $e_{i}^{*} e_{j}^{*}=0$ if $i \neq j$. Select an element $e_{j} \in \mathfrak{X}$ such that its residue class $\bmod \mathfrak{N}$ is $e_{j}^{*}$. Then $\pi\left(e_{j}\right) V=\pi\left(e_{j}^{*}\right) V=V_{\mathfrak{D}_{j}}$. Therefore if $\psi \in V_{\mathfrak{D}_{j}}, \psi=\pi\left(e_{j}\right) \phi$ $(\phi \in V)$. Hence $\pi\left(e_{i}\right) \psi=\pi\left(e_{i}^{*} e_{j}^{*}\right) \phi=\delta_{i j} \pi\left(e_{j}^{*}\right) \phi=\delta_{i j} \psi$. This proves the lemma.

Now we return to Theorem 2. Put $M=M\left(\mathfrak{D}_{0}, \chi\right)$ and $\mathfrak{N}=\mathfrak{N}_{\mathfrak{D}_{0}}$ for brevity. Let $b \rightarrow b^{*}$ denote the natural mapping and $\pi$ the natural representation of $\mathscr{B}$ on $\mathfrak{B}^{*}=\mathfrak{B} /\left(\mathfrak{B} \mathfrak{N}+\mathfrak{B} \mathfrak{Z}_{x}\right)$. Let $A$ be the set of all $a \in \mathfrak{B}$ such that $\mathfrak{N} a \subset \mathfrak{B N}$ $+\mathfrak{B} \mathfrak{Z}_{x}$. Then $A$ is a subalgebra of $\mathfrak{B}$ and $A \supset \mathfrak{D} \mathfrak{X}+\mathfrak{B} \mathfrak{N}+\mathfrak{B} \mathfrak{B}_{x}$. Let $\mathfrak{B}_{\mathfrak{D}}^{*}$ denote the set of all elements $b^{*} \in \mathfrak{B}^{*}$ which transform under $\pi(\mathfrak{l})$ according to $\mathfrak{D}(\mathfrak{D} \in \Omega)$. Then it is obvious that $\mathfrak{B}_{\mathfrak{D}_{0}}^{*}=A^{*}=A / \mathfrak{B N}+\mathfrak{B} \mathfrak{B}_{x}$.

Let $\mathfrak{M} \in M$. Denote by $b \rightarrow \bar{b}$ the natural mapping and by $\bar{\pi}$ the natural representation of $\mathfrak{B}$ on $\overline{\mathfrak{B}}=\mathfrak{B} / \mathfrak{M}$. Since $\mathfrak{M} \supset \mathfrak{B N}+\mathfrak{B} \mathfrak{B}$ x we may identify $\overline{\mathfrak{B}}$ with $\mathfrak{B}^{*} / \mathfrak{M}^{*}$ in a natural way $\left(\mathfrak{M}^{*}=\mathfrak{M} / \mathfrak{B} \mathfrak{N}+\mathfrak{B} \mathfrak{B}_{x}\right)$. Let $b^{*} \rightarrow \overline{b^{*}}$ denote the natural mapping of $\mathfrak{B}^{*}$ on $\overline{\mathfrak{B}}=\mathfrak{B}^{*} / \mathfrak{M}^{*}$. Then if $\overline{\mathfrak{B}}_{\mathfrak{D}}$ is the set of all elements in $\overline{\mathfrak{B}}$ which transform under $\bar{\pi}(\mathfrak{l})$ according to $\mathfrak{D}(\mathfrak{D} \in \Omega)$, it is obvious that

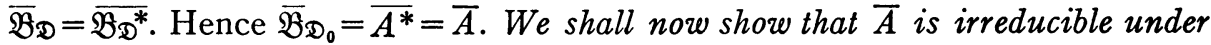
$\bar{\pi}(A)$. Let $a_{0}, a_{1}$ be two elements in $A$ such that $\bar{a}_{0} \neq 0$. Since $\mathfrak{M}$ is maximal, $\overline{\mathfrak{B}}$ is irreducible under $\bar{\pi}(\mathfrak{B})$ and therefore there exists an element $b_{0} \in \mathfrak{B}$ such that $\bar{\pi}\left(b_{0}\right) \bar{a}_{0}=\bar{a}_{1}$. Since $\mathfrak{B}^{*}=\sum_{\mathfrak{I} \in \Omega} \mathfrak{B}_{\mathfrak{D}}^{*}$ and $\operatorname{dim} \mathfrak{B}_{\mathfrak{D}}^{*}<\infty$ (see Theorem 1 of [5]), it follows that there exists a finite number of distinct elements $\mathfrak{D}_{1}, \cdots, \mathfrak{D}_{N} \in \Omega\left(\mathfrak{D}_{j} \neq \mathfrak{D}_{0}, 1 \leqq j \leqq N\right)$ such that $\pi\left(b_{0}\right) \mathfrak{B}_{\mathfrak{D}_{0}}^{*} \subset \sum_{0 \leqq j \leqq N} \mathfrak{B}_{\mathfrak{D}_{j}}^{*}$. From Lemma 4 we can find an element $e \in \mathfrak{X}$ such that $\pi(e) a^{*}=a^{*}$ or 0 according as $a^{*} \in \mathfrak{B}_{\mathfrak{D}_{0}}^{*}$ or $a^{*} \in \mathfrak{B}_{\mathfrak{D}_{j}}^{*}, 1 \leqq j \leqq N$. Then if we put $b=e b_{0}, \pi(b) \mathfrak{B}_{\mathfrak{D}_{0}}^{*} \subset \mathfrak{B}_{\mathfrak{D}_{0}}^{*}$ and $\bar{\pi}(b) \bar{a}_{0}=\bar{\pi}(e) \bar{a}_{1}=\bar{a}_{1}$. Since $\pi(b) \mathfrak{B}_{\mathfrak{D}_{0}}^{*} \subset \mathfrak{B}_{\mathfrak{D}_{0}}^{*}, b \in A$ and hence $\bar{a}_{1} \in \bar{\pi}(A) \bar{a}_{0}$. This shows that $\bar{A}$ is irreducible under $\bar{\pi}(A)$. Since $1 \notin \mathfrak{M}, \mathfrak{M} \cap A \neq A$ and therefore $A \cap \mathfrak{M}$ is maximal in $A$.

Let $M_{A}$ be the set of all maximal ideals in $A$ which contain $\mathfrak{B N}+\mathfrak{B} \mathfrak{Z}_{x}$. We have just seen that if $\mathfrak{M} \in M$ then $\mathfrak{M} \cap A \in M_{A}$. We shall now show that the mapping $\mathfrak{M} \rightarrow \mathfrak{M} \cap A(\mathfrak{M} \in M)$ is a 1-1 mapping of $M$ onto $M_{A}$. (This is actually more than we need for Theorem 2.) Let $\mathfrak{M}$ and $\mathfrak{M}^{\prime}$ be two distinct elements in $M$. Since they are both maximal, $\mathfrak{M}+\mathfrak{M}^{\prime}=\mathfrak{B}$ and therefore we can select $b \in \mathfrak{M}$ and $b^{\prime} \in \mathfrak{M}^{\prime}$ such that $b+b^{\prime}=1$. Choose a finite set of distinct classes $\mathfrak{D}_{1}, \cdots, \mathfrak{D}_{N}$ in $\Omega\left(\mathfrak{D}_{0} \neq \mathfrak{D}_{j}, 1 \leqq j \leqq N\right)$ such that $\pi(b) \mathfrak{B}_{\mathfrak{D}_{0}}^{*}$ and $\pi\left(b^{\prime}\right) \mathfrak{B}_{\mathfrak{D}}^{*}$ are both contained in $\sum_{j=0}^{N} \mathfrak{B}_{\mathfrak{D}_{j}}^{*}$. In accordance with Lemma 4 we 
can pick an element $e \in \mathfrak{X}$ such that $\pi(e) \mathfrak{B}_{\mathfrak{D}_{i}}^{*}=\{0\}, 1 \leqq j \leqq N$, and $\pi(e) a^{*}=a^{*}$ if $a^{*} \in \mathfrak{B}_{\mathfrak{D}_{0}}^{*}$. Then if $a=e b$ and $a^{\prime}=e b^{\prime}, a^{*}+\left(a^{\prime}\right)^{*}=\pi(e) 1^{*}=1^{*}$ since $1^{*} \in \mathfrak{B}_{\mathfrak{D}_{0}}^{*}$. It is clear that $a \in \mathfrak{M} \cap A$ and $a^{\prime} \in \mathfrak{M}^{\prime} \cap A$. Since $\mathfrak{M} \cap \mathfrak{M}^{\prime} \cap A \supset \mathfrak{B} \mathfrak{R}+\mathfrak{B} \mathfrak{B}_{x}$ it follows that $\mathfrak{M} \cap A+\mathfrak{M}^{\prime} \cap A=A$ and therefore $\mathfrak{M} \cap A \neq \mathfrak{M}^{\prime} \cap A$. Now let $B$ be any element in $M_{A}$. Put $\mathfrak{M}_{0}=\mathfrak{B} B$. We claim $\mathfrak{M}_{0} \neq \mathfrak{B}$. For otherwise $\mathfrak{B}^{*}=\pi(\mathfrak{B}) B^{*}$ where $B^{*}$ is the image of $B$ in $\mathfrak{B}^{*}$. Hence $1^{*}=\sum_{i=1}^{r} \pi\left(b_{i}\right) u_{i}{ }^{*}$ $\left(b_{i} \in \mathfrak{B}, u_{i}^{*} \in B^{*}\right)$. Again we may choose a finite set of classes $\mathfrak{D}_{1}, \cdots, \mathfrak{D}_{N}$ in $\Omega\left(\mathfrak{D}_{j} \neq \mathfrak{D}_{0}, 1 \leqq j \leqq N\right)$ such that $\pi\left(b_{i}\right) \mathfrak{B}_{\mathfrak{D}_{0}}^{*} \subset \sum_{0 \leqq j \leqq N} \mathfrak{B}_{\mathfrak{D}_{j}}^{*}(1 \leqq i \leqq r)$. Now select $e \in \mathfrak{X}$ such that $\pi(e) \mathfrak{B}_{\mathfrak{D}_{j}}^{*}=\{0\}, 1 \leqq j \leqq N$, and $\pi(e) a^{*}=a^{*}$ if $a^{*} \in \mathfrak{B}_{\mathfrak{D}_{0}}^{*}$. Then

$$
1^{*}=\pi(e) 1^{*}=\sum_{i=1}^{r} \pi\left(e b_{i}\right) u_{i}^{*} .
$$

Put $e b_{i}=a_{i}$ and let $u_{i}$ be an element in $B$ such that its image in $B^{*}$ is $u_{i}{ }^{*}$. Then since $\pi\left(a_{i}\right) \mathfrak{B}_{\mathfrak{D}_{0}}^{*} \subset \mathfrak{B}_{\mathfrak{D}_{0}}^{*}, a_{i} \in A$, and therefore $\sum_{i=1}^{r} a_{i} u_{i} \in B$. On the other hand

$$
1^{*}=\left(\sum_{i=1}^{r} a_{i} u_{i}\right) *
$$

and $B \supset \mathfrak{B N}+\mathfrak{B} \mathfrak{B}_{x}$. Therefore $B=A$ and we get a contradiction with the fact that $B \in M_{A}$. Hence $\mathfrak{M}_{0} \neq \mathfrak{B}$ and therefore by Zorn's lemma we can find a maximal left ideal $\mathfrak{M}$ in $\mathfrak{B}$ which contains $\mathfrak{M}_{0}$. Then $\mathfrak{M} \in M$ and $\mathfrak{M} \cap A$ is a maximal left ideal in $A$ containing $B$. Since $B$ is maximal, $\mathfrak{M} \cap A=B$. This proves that the mapping $\mathfrak{M} \rightarrow \mathfrak{M} \cap A(\mathfrak{M} \in M)$ is a $1-1$ mapping of $M$ onto $M_{A}$.

Now suppose $\mathfrak{M}_{1}, \mathfrak{M}_{2}$ are two elements in $M$. Put $B_{i}=\mathfrak{M}_{i} \cap A(i=1,2)$. We shall prove that $\mathfrak{M}_{1}$ and $\mathfrak{M}_{2}$ are equivalent in $\mathfrak{B}$ if and only if $B_{1}, B_{2}$ are equivalent in $A$. Suppose $\mathfrak{M}_{1}$ and $\mathfrak{M}_{2}$ are equivalent. Then we can pick an element $b_{0} \in \mathfrak{B}$ such that $b b_{0} \in \mathfrak{M}_{2}(b \in \mathfrak{B})$ if and only if $b \in \mathfrak{M}_{1}$. Choose $\mathfrak{D}_{1}, \cdots, \mathfrak{D}_{N} \in \Omega\left(\mathfrak{D}_{j} \neq \mathfrak{D}_{0}, 1 \leqq j \leqq N\right)$ such that $\left(b_{0}\right) * \in \sum_{0 \leqq j \leqq N} \mathfrak{B}_{\mathfrak{D}_{j}}^{*}$ and let $e$ be an element in $\mathfrak{X}$ such that $\pi(e-1) \mathfrak{B}_{\mathfrak{D}_{0}}^{*}=\{0\}$ and $\pi(e) \mathfrak{B}_{\mathfrak{D}_{j}}^{*}=\{0\}(1 \leqq j \leqq N)$. Then if we put $a=e b_{0}, a \in A$. Moreover it is clear that $1-e \in \mathfrak{R} \subset \mathfrak{M}_{1}$ and therefore $b_{0}-a=(1-e) b_{0} \in \mathfrak{M}_{2}$. Therefore $b a \in \mathfrak{M}_{2}(b \in \mathfrak{B})$ if and only if $b \in \mathfrak{M}_{1}$. Hence $b a \in B_{2}(b \in A)$ if and only if $b \in B_{1}$. This proves that $B_{1}$ and $B_{2}$ are equivalent in $A$. Conversely let $B_{1}, B_{2}$ be two elements in $M_{A}$ which are equivalent in $A$. Then we can select an $a \in A$ such that $b a \in B_{2}(b \in A)$ if and only if $b \in B_{1}$. It is obvious that $a \notin B_{2}$. Let $\mathfrak{M}_{1}, \mathfrak{M}_{2}$ be the left ideals in $M$ such that $B_{i}=\mathfrak{M}_{i} \cap A(i=1,2)$ and let $\mathfrak{M}_{1}^{\prime}$ be the set of all elements $b$ in $\mathfrak{B}$ for which $b a \in \mathfrak{M}_{2}$. Since $\mathfrak{M}_{2}$ is maximal the natural representation of $\mathfrak{B}$ on $\mathfrak{B} / \mathfrak{M}_{2}$ is irreducible and therefore since $a \notin \mathfrak{M}_{2}$ it is clear that $\mathfrak{M}_{1}^{\prime}$ is a maximal left ideal equivalent to $\mathfrak{M}_{2}$. Moreover since $a \in A, a^{*} \in \mathfrak{B}_{\mathfrak{D}_{0}}^{*}$ and therefore $\mathfrak{M}_{1}^{\prime}$ $\supset \mathfrak{B N}+\mathfrak{B} \mathfrak{B}_{\mathbf{x}}$. Hence $\mathfrak{M}_{1}^{\prime} \in M$ and $\mathfrak{M}_{1}^{\prime} \cap A \supset B_{1}$. Since $B_{1}$ is maximal in $A$, $B_{1}=\mathfrak{M}_{1}^{\prime} \cap A$ and therefore $\mathfrak{M}_{1}^{\prime}=\mathfrak{M}_{1}$ in view of the result above. Hence $\mathfrak{M}_{1}$ 
and $\mathfrak{M}_{2}$ are equivalent and our assertion is proved.

Now let $\mathfrak{A}=\mathfrak{Q X}$. Then $A \supset \mathfrak{A}$. We shall prove that if $\mathfrak{M} \in M, \mathfrak{M} \cap \mathfrak{A}$ is maximal in $\mathfrak{A}$. Let $a \rightarrow \bar{a}$ be the natural mapping and $\nu$ the natural representation of $A$ on $A / \mathfrak{M} \cap A$. We know that $\operatorname{dim} \bar{A} \leqq \operatorname{dim} \mathfrak{B}_{\mathfrak{D}_{0}}^{*}<\infty$ and $\nu$ is irreducible. In order to prove that $\mathfrak{M} \cap \mathfrak{A}$ is maximal in $\mathfrak{A}$ it is sufficient to prove that $\bar{A}$ is irreducible under $\nu(\mathfrak{U})$. Since $\mathfrak{A} \supset \mathfrak{X}$ and $\bar{A}$ is semisimple under $\nu(\mathfrak{X})$ it would be enough to show that if $U$ and $V$ are any two subspaces of $\mathfrak{A}$ which are stable and simple under $\nu(\mathfrak{X})$, then $V \subset \nu(\mathfrak{H}) U$. It is obvious that the representations of $\mathfrak{X}$ induced on $V$ and $U$ lie in $\mathfrak{D}_{0}$. Hence there exists a linear isomorphism $\alpha$ of $U$ with $V$ such that $\nu(z) \alpha=\alpha \nu(z)(z \in \mathfrak{X})$. Since $\nu(A)$ is irreducible it follows from Burnside's Theorem that we can find an element $a \in A$ such that $\nu(a) u=\alpha u$ for all $u \in U$. Then $\nu(z a) u=\nu(z) \alpha u=\alpha \nu(z) u=\nu(a z) u$ $(u \in U, z \in \mathfrak{X})$. For any $X \in \mathfrak{l}$ let $\rho(X)$ denote the mapping $b \rightarrow[X, b](b \in A)$ of $A$ into itself. It is obvious that $\rho$ is a quasi semisimple representation of (and therefore of $\mathfrak{X}$ ) on $A$. Let $B$ be the set of all $b \in A$ such that $\nu(b) u=0$ for all $u \in U$. Then $\rho(X) a \in B(X \in \mathfrak{l})$ and it is obvious that $B$ is a left ideal in $A$ and $B \mathfrak{X} \subset B$. Hence $B$ is stable under $\rho(\mathfrak{X})$ and therefore $\rho(\mathfrak{X} \mathfrak{f}) a=\rho(\mathfrak{f} \mathfrak{X}) a \subset B$. Put $W=\rho(\mathfrak{X}) a$. Then $\mathfrak{Q} \cap W$ is the set of all elements $w \in W$ such that $\rho(X) w=0 \quad(X \in \mathfrak{l})$. Hence, from Lemma 7 of [5], $W=\mathfrak{Q} \cap W+\rho(\mathfrak{l}) W$. In particular $a \equiv q \bmod (\rho(\mathfrak{f}) W)$ where $q \in \mathfrak{Q} \cap W$. Since $\rho(\mathfrak{l}) W \subset B, \nu(a) u=\nu(q) u$ for all $u \in U$. Hence $V=\nu(a) U=\nu(q) U \subset \nu(\mathfrak{H}) U$ and our assertion is proved.

REMARK. We note for later use the fact that $\bar{A}=\nu(\mathfrak{I}) \overline{1}$ which follows from the irreducibility of $\bar{A}$ under $\nu(\mathfrak{A})$. If $\overline{\mathfrak{A}}$ is the image of $\mathfrak{A}$ in $\overline{\mathfrak{B}}=\mathfrak{B} / \mathfrak{M}$ under the natural mapping of $\mathfrak{B}$ on $\overline{\mathfrak{B}}$, then $\overline{\mathfrak{A}}=\overline{\mathfrak{B}}_{\mathfrak{D}_{0}}=\bar{A}$.

Let $\mathfrak{M}^{\prime}$ be another ideal in $M$ and let $\nu^{\prime}$ denote the natural representation of $A$ on $A / \mathfrak{M}^{\prime} \cap A$. If $\mathfrak{M}$ and $\mathfrak{M}^{\prime}$ are equivalent in $\mathfrak{B}$, the representations $\nu$ and $\nu^{\prime}$ of $A$ are equivalent. Therefore the representations of $\mathfrak{A}$ defined by $\nu$ and $\nu^{\prime}$ are also equivalent and this proves that $\mathfrak{M} \cap \mathfrak{A}$ and $\mathfrak{M}^{\prime} \cap \mathfrak{A}$ are equivalent in $\mathfrak{A}$. Conversely suppose $\mathfrak{M} \cap \mathfrak{A}$ and $\mathfrak{M}^{\prime} \cap \mathfrak{A}$ are equivalent in $\mathfrak{A}$. Then $\operatorname{sp} \nu(q)=\operatorname{sp} \nu^{\prime}(q)$ for all $q \in \mathfrak{A}$. Let $a$ be any element in $A$. Define $W=\rho(\mathfrak{X}) a$ as above. Then $W=W \cap \mathfrak{Q}+\rho(\mathfrak{f}) W$ and therefore $a \equiv q \bmod \rho(\mathfrak{f}) W$ where $q \in \mathfrak{Q}$. Now $\operatorname{sp}(\nu([X, b]))=\operatorname{sp}(\nu(X b-b X))=0(X \in \mathfrak{l}, b \in A)$. Hence $\operatorname{sp} \nu(a)=\operatorname{sp} \nu(q)$. Similarly $\operatorname{sp} \nu^{\prime}(a)=\operatorname{sp} \nu^{\prime}(q)$. Therefore $\operatorname{sp} \nu(a)=\operatorname{sp} \nu^{\prime}(a)$ for all $a \in A$ and then it follows immediately from the theory of semisimple representations of an associative algebra (see Appendix, Lemma 16) that $\nu$ and $\nu^{\prime}$ are equivalent. Hence $\mathfrak{M} \cap A$ and $\mathfrak{M}^{\prime} \cap A$ are equivalent in $A$. In view of the result proved above this implies that $\mathfrak{M}$ and $\mathfrak{M}^{\prime}$ are equivalent in $\mathfrak{B}$. Hence Theorem 2 is established.

We have established above a 1-1 equivalence-preserving correspondence between elements of $M$ and the maximal left ideals in $A^{*}=A / \mathfrak{B N}+\mathfrak{B} \mathfrak{B}_{x}$. (We observe that $\mathfrak{B N}+\mathfrak{B} 乃_{\chi}$ is a two-sided ideal in $A$ and therefore $A^{*}$ is an algebra.) Since $\operatorname{dim} A^{*}<\infty, A^{*}$ has only a finite number of inequivalent irreducible representations. Therefore we get the following corollary. 
CoRollary 1. $M\left(\mathfrak{D}_{0}, \chi\right)$ contains only a finite number of inequivalent left ideals.

This is the generalisation of a result which has been proved in an earlier paper (Theorem 4 of [3]).

Let us call a representation $\pi$ of $\mathfrak{B}$ on $V$ quasisimple if it maps every element of $B$ into a scalar multiple of the unit operator and if the representation $X \rightarrow \pi(X)(X \in \mathfrak{f})$ is quasi semisimple (see $[5, \S 2])$. We denote by $V_{\mathscr{D}}$ $(\mathfrak{D} \in \Omega)$ the set of those elements of $V$ which transform under $\pi(\mathfrak{l})$ according to $\mathfrak{D}$.

COROLlaRy 2. Let $\pi_{1}, \pi_{2}$ be two quasisimple irreducible representations of $\mathfrak{B}$ on the vector spaces $V_{1}, V_{2}$ respectively. Choose a class $\mathfrak{D}_{i} \in \Omega$ which occurs in $\pi_{i}$ and let $\nu_{i}$ denote the representation of $\mathfrak{A}=\mathfrak{Q X X}$ on $V_{1, D_{i}}$ induced under $\pi_{i}(i=1,2)$. Then $\nu_{1}, \nu_{2}$ are both irreducible. Moreover, if they are equivalent the same holds for $\pi_{1}, \pi_{2}$.

Let $\chi_{i}$ be the homomorphism of 8 into $C$ such that $\pi_{i}\left(z-\chi_{i}(z)\right)=0$ $(z \in 3)$. We have seen above that $\operatorname{dim} V_{i, D_{i}}<\infty$ and $\nu_{i}$ is irreducible. Now if $\nu_{1}, \nu_{2}$ are equivalent, $\chi_{1}=\chi_{2}$ and $\mathfrak{D}_{1}=\mathfrak{D}_{2}$. Therefore Theorem 2 is applicable and we conclude that $\pi_{1}$ is equivalent to $\pi_{2}$.

3. Some auxiliary results. In this section we obtain two results whose full significance will appear in a subsequent paper. We keep to the above notation.

THEOREM 3. Let $\mathfrak{M}$ be a left ideal in $M\left(\mathfrak{D}_{0}, \chi\right)$ and $\pi$ the natural representation of $\mathfrak{B}$ on $\mathfrak{B}^{*}=\mathfrak{B} / \mathfrak{M}$. For any $\mathfrak{D} \in \Omega$ let $\mathfrak{B}_{\mathfrak{D}}^{*}$ denote the set of elements in $\mathfrak{B}^{*}$ which transform under $\pi(\mathfrak{X})$ according to $\mathfrak{D}$. Then there exists an integer $N$ such that

$$
\operatorname{dim} \mathfrak{B}^{*} \leqq N d(\mathfrak{D})^{2}
$$

where $d(\mathfrak{D})$ is the degree of any representation in $\mathfrak{D}$.

The proof of this theorem is based on an unpublished result of Chevalley. Consider the symmetric algebra $S(\mathfrak{p})$ over $\mathfrak{p}$. For any $X \in \mathfrak{f}$ define a derivation $d_{X}$ of $S(\mathfrak{p})$ by the rule $d_{X} Y=[X, Y](Y \in \mathfrak{p})$. Let $\Im$ be the set of those $f \in S(\mathfrak{p})$ for which $d_{X} f=0$ for all $X \in \mathfrak{f}$. Let $\sigma$ be a simple representation of $\mathfrak{t}$ on a space $U$ of dimension $d$. Consider the Kronecker product $S(\mathfrak{p}) \times U$. Let $S_{m}(\mathfrak{p})$ denote the set of homogeneous elements in $S(\mathfrak{p})$ of degree $m$. We shall say that an element in $S(\mathfrak{p}) \times U$ is homogeneous of degree $m$ if it lies in $S_{m}(\mathfrak{p}) \times U$. We turn $S(\mathfrak{p}) \times U$ into an $S(\mathfrak{p})$-module by defining $f(g \times u)=f g \times u(f, g \in S(\mathfrak{p})$, $u \in U)$. Let $\nu$ be the representation of $\mathfrak{t}$ on $S(\mathfrak{p}) \times U$ defined as follows:

$$
\nu(X)(f \times u)=\left(d_{X} f\right) \times u+f \times \sigma(X) u \quad(X \in \mathfrak{k}, f \in S(\mathfrak{p}), u \in U) .
$$

An element $e \in S(\mathfrak{p}) \times U$ is called an invariant if $\nu(X) e=0$ for all $X \in \mathfrak{l}$. Let 
$\Re$ be the set of all invariants. Then it is obvious that $\Re$ is a module over $\Im$.

Chevalley's Theorem. We can choose $2 d$ homogeneous elements $e_{1}, e_{2}, \cdots$, $e_{2 d}$ in $\Re$ such that $\Re=\sum_{1 \leqq i \leqq 2 d} \Im e_{i}$.

COROLlary. The above result holds also if $\sigma$ instead of being simple is semisimple.

Let $U=\sum_{1 \leqq k \leqq s} U_{k}$ where each $U_{k}$ is stable and simple under $\sigma$ and the sum is direct. Then $\Re=\sum_{1 \leqq k \leqq s} \Re_{k}$ where $\Re_{k}=\Re \cap\left(S(\mathfrak{p}) \times U_{k}\right)$. If we apply the above theorem to each $\Re_{k}$ we get the result.

We shall now make use of Chevalley's theorem to prove Theorem 3 . Our method is the same as that used in $\S 2$ of [5]. Let $S(\mathfrak{g})$ be the symmetric algebra over $\mathfrak{g}$. For any $X \in \mathfrak{g}$ define a derivation $D_{X}$ of $S(\mathfrak{g})$ by the rule $D_{X} Y=[X, Y](Y \in \mathfrak{g})$. Let $J$ be the set of $F \in S(\mathfrak{g})$ for which $D_{X} F=0$ for all $X \in \mathfrak{g}$. For any $F \in J$ we denote by $F_{\mathfrak{p}}$ the restriction of $F$ on $\mathfrak{p}$ (see [5, end of §2]). Let $J_{\mathfrak{p}}$ be the image of $J$ under this mapping. Then we know from $[5, \S 3]$ that $\Im$ is a finite $J_{\mathfrak{p}}$-module and therefore we may select a finite set of homogeneous elements $\omega_{\beta}, 1 \leqq \beta \leqq \alpha$, in $\Im$ such that $\Im=\sum_{1 \leqq \beta \leqq \alpha} J_{p} \omega_{\beta}$. Since $\mathfrak{M} \in M\left(\mathfrak{D}_{0}, \chi\right)$ it is clear that $\mathfrak{B}_{\mathfrak{D}_{0}}^{*} \neq\{0\}$. Choose an element $\psi_{0} \in \mathfrak{B}_{\mathfrak{D}_{0}}^{*}$ $\left(\psi_{0} \neq 0\right)$ such that $U=\pi(\mathfrak{X}) \psi_{0}$ is irreducible under $\pi(\mathfrak{X})$. Let $\psi_{i}, 1 \leqq i \leqq d_{0}$, be a base for $U$. Let $\mathfrak{D}$ be any class in $\Omega$. Put $d=d(\mathfrak{D})$ and choose a representation $\sigma$ of $\mathfrak{f}$ on a vector space $V$ of dimension $d$ such that $\sigma$ is dual (or contragredient) to any representation in $\mathfrak{D}$. We consider the space $S(\mathfrak{p}) \times U \times V$ and the set $\Re$ of all invariants in it. Then in view of the above corollary we can find $2 d d_{0}$ homogeneous elements $e_{k}\left(1 \leqq k \leqq 2 d d_{0}\right)$ in $\Re$ such that

$$
\Re=\sum_{1 \leqq k \leqq 2 d d_{0}} \Im e_{k}^{\prime}=\sum_{1 \leqq k \leqq 2 d d_{0}} \sum_{1 \leqq \beta \leqq \alpha} J_{p} \omega_{\beta} e_{k}^{\prime} .
$$

Put $N=2 \alpha d_{0}$ and the elements $\omega_{\beta} e_{k}^{\prime}\left(1 \leqq \beta \leqq \alpha, 1 \leqq k \leqq 2 d d_{0}\right)$ in a sequence $e_{\beta}(1 \leqq \beta \leqq N d)$. Then $e_{\beta}$ are homogeneous elements in $\Re$ and $\Re=\sum_{1 \leqq \beta \leqq N d} J_{\mathrm{p}} e_{\beta}$. Let $v_{j}, 1 \leqq j \leqq d$, be a base for $V$ and let

$$
e_{\beta}=\sum_{1 \leqq j \leqq d} e_{\beta, j} \times v_{j}
$$

where $e_{\beta, j} \in S(\mathfrak{p}) \times U$. Let $\lambda$ denote the canonical mapping of $S(\mathfrak{g})$ into $\mathfrak{B}$ (see $[5, \S 2])$. We define a linear mapping $\Gamma$ of $S(\mathfrak{p}) \times U$ into $\mathfrak{B}^{*}$ as follows:

$$
\Gamma(f \times u)=\pi(\lambda(f)) u \quad(f \in S(\mathfrak{p}), u \in U) .
$$

We intend to show that the $N d^{2}$ elements $\Gamma\left(e_{\beta, j}\right)(1 \leqq \beta \leqq N d, 1 \leqq j \leqq d)$ span $\mathfrak{B}_{\mathfrak{D}}^{*}$. Since $N$ is independent of $\mathfrak{D}$ this would prove Theorem 3 .

First of all we claim that $\Gamma\left(e_{\beta, j}\right) \in \mathfrak{B}_{\mathfrak{D}}^{*}$. Let $\nu$ be the representation of $\mathfrak{l}$ on $S(\mathfrak{p}) \times U$ defined as follows:

$$
\nu(X)(f \times u)=\left(d_{X} f\right) \times u+f \times \pi(X) u \quad(f \in S(\mathfrak{p}), u \in U, X \in \mathfrak{t}) .
$$


Since $\lambda\left(d_{X} f\right)=[X, \lambda(f)]$ (see Lemma 11 of [5]) it follows that $\Gamma(\nu(X)(f \mathbf{X} u)$ ) $=\pi(X) \Gamma(f \times u)$. Now consider the element $e_{\beta}$ in $S(\mathfrak{p}) \times U \times V$. If $e_{\beta} \neq 0$ the representation of $\mathfrak{l}$ induced under $\nu$ on the space $W_{\beta}$ spanned by $e_{\beta, j}$ $(1 \leqq j \leqq d)$ is clearly contragredient to $\sigma$. On the other hand if $e_{\beta}=0, W_{\beta}=\{0\}$ and therefore in either case $\Gamma\left(W_{\beta}\right) \subset \mathfrak{B}_{\mathfrak{D}}^{*}$. Hence $\Gamma\left(e_{\beta, j}\right) \in \mathfrak{B}_{\mathfrak{D}}^{*}, 1 \leqq \beta \leqq N d$, $1 \leqq j \leqq d$. Now put $\mathfrak{B}_{m}^{*}=\Gamma\left(S_{m}(\mathfrak{p}) \times U\right)(m \geqq 0)$. Since $\mathfrak{B}=\mathfrak{B X}$ where $\mathfrak{P}=\lambda(S(\mathfrak{p}))$, it is obvious that $\mathfrak{B}^{*}=\pi(\mathfrak{B}) U=\Gamma(S(\mathfrak{p}) \times U)$. Hence $\mathfrak{B}^{*}=\sum_{m \geqq 0} \mathfrak{B}_{m}^{*}$. Moreover since $S_{m}(\mathfrak{p}) \times U$ is stable under $\nu(\mathfrak{l})$ the same holds for $\mathfrak{B}_{m}^{*}$ under $\pi(\mathfrak{l})$. Also we know that the natural representation of $\mathfrak{t}$ on $\mathfrak{B}^{*}$ is quasi semisimple (see Theorem 1 of [5]) and so it follows (see Lemma 6 of [5]) that $\mathfrak{B}_{m}^{*}$ $=\sum_{\mathcal{D}^{\prime} \in \Omega} \mathfrak{B}_{m}^{*} \cap \mathfrak{B}_{\mathcal{D}^{\prime}}^{*}$. Therefore

$$
\sum_{\mathfrak{D}^{\prime} \in \Omega} \mathfrak{B}_{\mathfrak{D}^{\prime}}^{*}=\mathfrak{B}^{*}=\sum_{m \geqq 0} \mathfrak{B}_{m}^{*}=\sum_{m \geqq 0} \sum_{\mathfrak{D}^{\prime} \in \Omega} \mathfrak{B}_{m}^{*} \cap \mathfrak{B}_{\mathfrak{D}^{\prime}}^{*}=\sum_{\mathcal{D}^{\prime} \in \Omega} \sum_{m \geqq 0} \mathfrak{B}_{m}^{*} \cap \mathfrak{B}_{\mathfrak{D}^{\prime}}^{*}
$$

Since the sum on the left-hand side is direct we conclude that

$$
\mathfrak{B}_{\mathfrak{D}}^{*}=\sum_{m \geqq 0}\left(\mathfrak{B}_{m}^{*} \cap \mathfrak{B}_{\mathfrak{D}}^{*}\right) \text {. }
$$

Hence if $A=\sum_{\beta, j} C \Gamma\left(e_{\beta, j}\right)$ it would be sufficient to prove that

$$
\mathfrak{B}_{m}^{*} \cap \mathfrak{B}_{\mathfrak{D}}^{*} \subset A
$$

Put $\mathfrak{B}_{-1}^{*}=\{0\}$. Then the above statement is true for $m=-1$. Hence we may use induction on $m$ and assume that $m \geqq 0$. Let $z^{*}$ be an element in $\mathfrak{B}_{m}^{*} \cap \mathfrak{B}_{\mathfrak{D}}^{*}$. We have to show that $z^{*} \in A$. The representation of $\mathfrak{X}$ induced on the space $\pi(\mathfrak{X}) z^{*}$ is semisimple. Hence it would be enough to prove that if $W$ is any simple subspace of $\pi(\mathfrak{X}) z^{*}$ then $W \subset A$. It is obvious that the representation of $\mathfrak{t}$ induced on $W$ lies in $\mathfrak{D}$. Hence we can choose a base $w_{1}^{*}, \cdots, w_{d}^{*}$ for $W$ such that

$$
\xi(X)\left(\sum_{1 \leqq j \leqq d} w_{j}^{*} \times v_{j}\right)=0
$$

where $\xi$ is the representation of $\mathfrak{t}$ induced on the Kronecker product $W \times V$. Since $W \subset \mathfrak{B}_{m}^{*} \cap \mathfrak{B}_{\mathfrak{D}}^{*}$ and since $S_{m}(\mathfrak{p}) \times U$ is stable and semisimple under $\nu(\mathfrak{X})$, it is easily seen that we can select elements $w_{j} \in S_{m}(\mathfrak{p}) \times U$ such that $\Gamma\left(w_{j}\right)=w_{j}^{*}$ and the space spanned by $w_{j}, i \leqq j \leqq d$, is invariant and irreducible under $\nu(\mathfrak{X})$. Then it is obvious that $\sum_{1 \leqq j \leqq d} w_{j} X v_{j}$ belongs to $\Re$ and therefore

$$
\sum_{j} w_{j} \times v_{j}=\sum_{1 \leqq \beta \leqq N d} f_{\beta} e_{\beta}
$$

Since $w_{j}^{*} \neq 0$ not all $e_{\beta}$ can be zero. Suppose $e_{\beta} \neq 0$ for $1 \leqq \beta \leqq \beta_{0}$ and $e_{\beta}=0$ for $\beta_{0}<\beta \leqq N d\left(1 \leqq \beta_{0} \leqq N d\right)$. Let $\delta_{\beta}$ be the degree of $e_{\beta}\left(1 \leqq \beta \leqq \beta_{0}\right)$. It is clear that the homogeneous components of any element in $J_{\mathfrak{p}}$ or $J$ also belong to $J_{\mathfrak{p}}$ or 
$J$ respectively. Hence we may assume that $f_{\beta}$ is either zero or homogeneous of degree $m-\delta_{\beta}$. We may assume that $f_{\beta} \neq 0$ if $1 \leqq \beta \leqq \beta_{1}$ and $f_{\beta}=0$ if $\beta_{1}<\beta \leqq \beta_{0}$ $\left(1 \leqq \beta_{1} \leqq \beta_{0}\right)$. Choose an element $F_{\beta} \in J$ such that $f_{\beta}=\left(F_{\beta}\right)_{\mathfrak{p}}$. Clearly we may assume that $F_{\beta}$ is homogeneous of degree $m_{\beta}=m-\delta_{\beta}\left(1 \leqq \beta \leqq \beta_{1}\right)$. But then $F_{\beta}-f_{\beta} \in \sum_{m_{\beta}>\mu \geqq 0} S_{\mu}(\mathfrak{p}) S(\mathfrak{f})$ where $S(\mathfrak{l})$ is the symmetric algebra over $\mathfrak{f}$.

Now put

$$
w_{j}^{* \prime}=\sum_{1 \leqq \beta \leqq \beta_{1}} \pi\left(\lambda\left(F_{\beta}\right)\right) \Gamma\left(e_{\beta, j}\right) .
$$

Since $F_{\beta} \in J, \lambda\left(F_{\beta}\right) \in Z$ (see the corollary to Lemma 11 of [5]) and therefore

$$
w_{j}^{* \prime}=\sum_{1 \leqq \beta \leqq \beta_{1}} \chi\left(\lambda\left(F_{\beta}\right)\right) \Gamma\left(e_{\beta, j}\right) \in A .
$$

Hence $w_{j}^{*}-w_{j}^{* \prime} \in \mathfrak{B}_{\mathfrak{D}}^{*}$. Moreover

$$
w_{j}^{*}-w_{j}^{* \prime}=\sum_{1 \leqq \beta \leqq \beta_{1}}\left\{\Gamma\left(f_{\beta} e_{\beta, j}\right)-\pi\left(\lambda\left(F_{\beta}\right)\right) \Gamma\left(e_{\beta, j}\right)\right\},
$$

$\Gamma\left(f_{\beta} e_{\beta, j}\right)-\pi\left(\lambda\left(F_{\beta}\right)\right) \Gamma\left(e_{\beta, j}\right)$

$$
=\left\{\Gamma\left(f_{\beta} e_{\beta, j}\right)-\pi\left(\lambda\left(f_{\beta}\right)\right) \Gamma\left(e_{\beta, j}\right)\right\}-\pi\left(\lambda\left(F_{\beta}-f_{\beta}\right)\right) \Gamma\left(e_{\beta, j}\right) .
$$

Since $F_{\beta}-f_{\beta} \in \sum_{m_{\beta}>\mu \geqq 0} S_{\mu}(\mathfrak{p}) S(\mathfrak{t})\left(1 \leqq \beta \leqq \beta_{1}\right)$ and $\Gamma\left(e_{\beta, j}\right) \in \mathfrak{B}_{\delta_{\beta}}^{*}$ it follows that

$$
\begin{aligned}
& \pi\left(\lambda\left(F_{\beta}-f_{\beta}\right)\right) \Gamma\left(e_{\beta, j}\right) \in \sum_{m_{\beta}>\mu \geqq 0} \pi\left(\lambda\left(S_{\mu}(\mathfrak{p})\right) \mathfrak{B}_{\delta_{\beta}}^{*}\right. \\
& =\sum_{m_{\beta}>\mu \geqq 0} \pi\left(\lambda\left(S_{\mu}(\mathfrak{p})\right) \lambda\left(S_{\delta_{\beta}}(\mathfrak{p})\right) U \subset \sum_{m>\mu \geqq 0} \mathfrak{B}_{\mu}^{*}\right.
\end{aligned}
$$

since

$$
\lambda\left(S_{\mu}(\mathfrak{p})\right) \lambda\left(S_{\delta_{\beta}}(\mathfrak{p})\right) \subset \sum_{\mu+\delta_{\beta} \geqq \mu_{1} \geq 0} \lambda\left(S_{\mu_{1}}(\mathfrak{p})\right) \mathfrak{X}
$$

from Lemma 13 of [5]. Moreover

$$
\Gamma\left(f_{\beta} e_{\beta, j}\right)-\pi\left(\lambda\left(f_{\beta}\right)\right) \Gamma\left(e_{\beta, j}\right) \in \sum_{0 \leqq \mu<m} \pi\left(\lambda\left(S_{\mu}(\mathfrak{g})\right) U\right.
$$

(see the proof of Lemma 1 of [2]) where $S_{\mu}(\mathfrak{g})$ is the set of all homogeneous elements of $S(\mathfrak{g})$ of degree $\mu$. Since $\lambda\left(S_{\mu}(\mathfrak{g})\right) \subset \sum_{0 \leqq \mu_{1} \leqq \mu} \lambda\left(S_{\mu_{1}}(\mathfrak{p})\right) \mathfrak{X}$ it follows that

$$
\Gamma\left(f_{\beta} e_{\beta, j}\right)-\pi\left(\lambda\left(f_{\beta}\right)\right) \Gamma\left(e_{\beta, j}\right) \in \sum_{m>\mu \geqq 0} \mathfrak{B}_{\mu .}^{*}
$$

Hence

$$
w_{j}^{*}-w_{j}^{* \prime} \in \mathfrak{B}_{\mathfrak{D}}^{*} \cap\left(\sum_{m>\mu \geqq 0} \mathfrak{B}_{\mu}^{*}\right)=\sum_{m>\mu \geqq 0}\left(\mathfrak{B}_{\mu}^{*} \cap \mathfrak{B}_{\mathfrak{D}}^{*}\right) \subset A
$$


by induction hypothesis. Since $w_{j}^{* \prime} \in A$ it follows that $w_{j}{ }^{*} \in A$ and therefore $W \subset A$. This proves our assertion and so the theorem is established.

We shall now prove a lemma which will be of use later.

Lemma 4. Let $\mathfrak{l}$ be a semisimple algebra over $C$ and $\mathfrak{U}$ the universal enveloping algebra of $\mathfrak{l}$. Then there exists an element $z$ in the center of $\mathfrak{U}$ such that if $\pi$ is any irreducible finite-dimensional representation of $\mathfrak{U}$ of degree $d$, then $\pi(z)=d^{2} \pi(1)$.

Let $\Gamma$ be a Cartan subalgebra of $\mathfrak{l}$ and $W$ the Weyl group of $\mathfrak{l}$ with respect to $\Gamma$. Let $l=\operatorname{dim} \Gamma$. We choose a fundamental system of roots $\left\{\alpha_{1}, \cdots, \alpha_{l}\right\}$ of $\mathfrak{l}$ (with respect to $\Gamma$ ) and introduce a lexicographic ordering in the set of all weights of finite-dimensional representations of $\mathfrak{l}$ with respect to this set (see [3, Part I] ). Let $B(X, Y)$ denote the bilinear form $\operatorname{sp}(\operatorname{ad} X$ ad $Y)$ on $\mathfrak{l}$ where $X \rightarrow \operatorname{ad} X$ is the adjoint representation of $\mathfrak{l}$. For every root $\alpha$ there exists a unique element $H_{\alpha} \in \Gamma$ such that $B\left(H, H_{\alpha}\right)=\alpha(H)$ for all $H \in \Gamma$. Let $2 \rho$ be the sum of all positive roots of $\mathfrak{l}$ and let $\pi$ be an irreducible finitedimensional representation of $\mathfrak{l}$ (or $\mathfrak{U}$ ) whose highest weight is $\Lambda$. Then we know that the degree of $\pi$ is

$$
d_{\Lambda}=\frac{\prod_{\alpha>0} \Lambda^{\prime}\left(H_{\alpha}\right)}{\prod_{\alpha>0} \rho\left(H_{\alpha}\right)}
$$

where $\Lambda^{\prime}=\Lambda+\rho$ (see Weyl [8]). Now we use the notation of Part III of [3]. Consider the polynomial

$$
f(x)=\left(\frac{\prod_{\alpha>0} x\left(H_{\alpha}\right)}{\prod_{\alpha>0} \rho\left(H_{\alpha}\right)}\right)^{2}
$$

in $x_{1}, \cdots, x_{l}$. It is clear that $s f=f$ for all $s \in W$. Hence it follows from Lemmas 38 and 39 of [3] that $f\left(x^{\prime}\right)=\chi_{x}(z)$ where $z$ is some element in the center of $\mathfrak{u}$. Hence $d_{\Lambda}^{2}=\chi_{\Lambda}(z)$. But we know that $\pi(z)=\chi_{\Lambda}(z) \pi(1)$ (see proof of Lemma 36 of [3]). Therefore $\pi(z)=d_{\Lambda}^{2} \pi(1)$ and this proves our assertion.

4. Proof of the main theorem. Let $G$ be the simply connected Lie group with the Lie algebra $g_{0}$. Let $K$ and $D$ be the analytic subgroups of $G$ corresponding to the subalgebras $\mathfrak{f}_{0}$ and $\mathfrak{c}_{0}\left(\mathfrak{c}_{0}\right.$ is the center of $\left.\mathfrak{f}_{0}\right)$. Let $Z$ be the center of $G$ and let $u \rightarrow u^{*}$ denote the natural mapping of $K$ on $K^{*}=K / D \cap Z$. We know that $K$ is simply connected and $K^{*}$ is compact (see for example Mostow [6]). Since there is a natural 1-1 correspondence between the finitedimensional simple representations of $K$ and those of $\mathfrak{t}$ we may regard any $\mathfrak{D} \in \Omega$ as a class of representations of $K$. Let $\Omega^{*}$ be the set of all equivalence classes of finite-dimensional simple representations of $K^{*}$. Since every representation $\sigma$ of $K^{*}$ can also be regarded as a representation $u \rightarrow \sigma\left(u^{*}\right)(u \in K)$ we may consider $\Omega^{*}$ as a subset of $\Omega$. 
Let $V$ be a vector space over $C$ and $y_{1}, \cdots, y_{r}$ a finite set of indeterminates. In $\S 1$ we have defined the space $V[y]$. Let $W$ be another vector space and $\beta$ a linear mapping of $V[y]$ into $W[y]$. We shall say that $\beta$ is $(y)$ linear if $\beta(f v)=f \beta(v)$ for $f \in C[y]$ and $v \in V[y]$. It is clear that any linear mapping of $V$ into $W$ can be extended uniquely to a $(y)$-linear mapping of $V[y]$ into $W[y]$. Similarly if $V$ is an algebra and $\pi$ is a representation of $V[y]$ on $W[y]$, then $\pi$ will be called $(y)$-linear if $\pi(f) w=f w$ for $f \in C[y]$ and $w \in W[y]$.

Let $\Gamma_{1}, \cdots, \Gamma_{r}$ be the base of $c$ introduced in $\S 1$ and let $y_{1}, \cdots, y_{r}$ be $r$ independent indeterminates. If $M$ is any integral linear function on $\mathrm{c}$ (see $\$ 1$ ) we put as before $\Gamma^{M}=\Gamma_{1}^{m_{1}} \Gamma_{2}^{m_{2}} \cdots \Gamma_{r}^{m_{r}}$ and $y^{M}=y_{1}^{m_{1}} \cdots y_{r}^{m_{r}}$ where $m_{i}$ $=M\left(\Gamma_{i}\right), 1 \leqq i \leqq r$. We shall now define two $(y)$-linear mappings $\beta_{y}$ and $\beta_{-y}$ of $\mathfrak{B}[y]$ into itself. It is sufficient to define $\beta_{y}(b)$ and $\beta_{-y}(b)$ for $b \in \mathfrak{B}$. We have seen in $\S 1$ that $b$ can be written uniquely in the form $b=\sum_{M} w_{M} \Gamma^{M}$ where $w_{M} \in \mathfrak{B X}$. (Here $\mathfrak{P}$ and $\mathfrak{X}^{\prime}$ have the same meaning as in $\S 1$.) Now we write $\beta_{y}(b)=\sum_{M} w_{M} \Gamma_{y}^{M}$ and $\beta_{-y}(b)=\sum_{M} w_{M} \Gamma_{-\nu}^{M}$ where

$$
\begin{aligned}
& \Gamma_{y}^{M}=\left(\Gamma_{1}+y_{1}\right)^{m_{1}}\left(\Gamma_{2}+y_{2}\right)^{m_{2}} \cdots\left(\Gamma_{r}+y_{r}\right)^{m_{r}}, \\
& \Gamma_{-y}^{M}=\left(\Gamma_{1}-y_{1}\right)^{m_{1}}\left(\Gamma_{2}-y_{2}\right)^{m_{2}} \cdots\left(\Gamma_{r}-y_{r}\right)^{m_{r}} \quad\left(m_{j}=M\left(\Gamma_{j}\right)\right) .
\end{aligned}
$$

It is obvious that $\beta_{y}\left(\beta_{-y}(b)\right)=\beta_{-y}\left(\beta_{y}(b)\right)=b$ for all $b \in \mathfrak{B}[y]$. Moreover since $\Gamma^{M}$ belongs to the center of $\mathfrak{X}$ it is clear that $\beta_{y}(b z)=\beta_{y}(b) \beta_{y}(z)$ for $b \in \mathfrak{B}$ and $z \in \mathfrak{X}$.

For any $x \in G$ and $v^{*} \in K^{*}$ we define $v_{x}^{*}, H\left(x, v^{*}\right), \Gamma\left(x, v^{*}\right)$ as in $\$ 11$ of [5]. Choose a base $Z_{1}, \cdots, Z_{n}$ for $g_{0}$ over $R$ and put $Z(t)=t_{1} Z_{1}+\cdots$ $+t_{n} Z_{n}\left(t_{j} \in R\right), x_{t}=\exp Z(t)$. Let $H_{1}, \cdots, H_{p}$ be the base for $\mathfrak{h}_{\mathfrak{p}_{0}}$ over $R$ which was selected in $\$ 2$ of [5]. Put

$$
\begin{aligned}
H\left(x_{t}^{-1}, v^{*}\right) & =\sum_{1 \leqq i \leqq p} H_{i} f_{i}\left(t, v^{*}\right), \\
\Gamma\left(x_{t}^{-1}, v^{*}\right) & =\sum_{1 \leqq j \leqq r} \Gamma_{j} g_{j}\left(t, v^{*}\right), \\
\chi D^{*}\left(\left(v_{x}^{*-1}\right)^{-1}\right) & =\chi\left(\mathfrak{D}^{*}, t, v^{*}\right)
\end{aligned}
$$

where $\chi_{\mathfrak{D}^{*}}$ is the character of $K^{*}$ corresponding to the class $\mathfrak{D}^{*} \in \Omega^{*}$. It is obvious (see the corollary to Lemma 26 of [5]) that $f_{i}\left(t, v^{*}\right), g_{j}\left(t, v^{*}\right)$, $\chi\left(\mathcal{D}^{*}, t, v^{*}\right)$ are all analytic functions on $R^{n} \times K^{*}$. Put $|t|=\max _{j}\left|t_{j}\right|$. We may choose $\epsilon>0$ so small that each of these functions can be expanded as a power series in $\left(t_{1}, \cdots, t_{n}\right)$ (with coefficients which are analytic functions on $K^{*}$ ) which is uniformly convergent for all $v^{*} \in K^{*}$ and $|t| \leqq \epsilon$ (see the proof of Lemma 29 of [5]). Let $\left(u_{1}, \cdots, u_{p}\right)$ be $p$ indeterminates independent of $\left(y_{1}, \cdots, y_{r}\right)$. If $H=\sum_{1 \leqq i \leqq p} a_{i} H_{i}, \Gamma=\sum_{1 \leqq j \leqq r} b_{j} \Gamma_{j}\left(a_{i}, b_{j} \in C\right)$ put $u(H)$ $=\sum_{1 \leqq i \leqq p} a_{i} u_{i}, y(\Gamma)=\sum_{1 \leqq j \leqq r} b_{j} y_{j}$. For any linear form $T$ in $(u, y)$ we define $e^{T}$ as the element $1+T+T^{2} / 2 !+T^{3} / 3 !+\cdots$ in the ring of power series in $(u, y)$ with coefficients in $C$. Then it is clear that the coefficients of the series 
$e^{-y\left(\Gamma\left(x t^{-1}, v^{*}\right)\right.} e^{-u\left(H\left(x_{t}^{-1}, v^{*}\right)\right)}$ are polynomials in $f_{i}\left(t, v^{*}\right)$ and $g_{j}\left(t, v^{*}\right)$. Moreover if $\Lambda$ and $\mu$ are any linear functions on $\mathfrak{h}_{\mathfrak{p}}$ and $\mathfrak{c}$ respectively, then the series resulting under the substitution $y \rightarrow \mu$ and $u \rightarrow \Lambda$ (that is, $y_{j} \rightarrow \mu\left(\Gamma_{j}\right)$ ), $1 \leqq j \leqq r$, and $u_{i}$ $\rightarrow \Lambda\left(H_{i}\right)(1 \leqq i \leqq p)$ converges absolutely to $e^{-\Lambda\left(H\left(x_{t}^{-1}, v^{*}\right)\right)} e^{-\mu\left(\Gamma\left(x_{t}{ }^{-1}, v^{*}\right)\right)}$. Let $2 \rho$ denote the sum of all positive roots of $\mathfrak{g}$ (with respect to $\mathfrak{h}$ ) under the ordering introduced in $\S 2$ of [5]. Consider linear functions $P, M, N$ on $\mathfrak{g}, \mathfrak{c}$, and $\mathfrak{h}_{\mathfrak{p}}$ respectively such that $P\left(Z_{i}\right)=p_{i}, M\left(\Gamma_{j}\right)=m_{j}, N\left(H_{k}\right)=n_{k}(1 \leqq i \leqq n, 1 \leqq j \leqq r, 1 \leqq k$ $\leqq p)$ are all non-negative integers. Put $P !=p_{1} ! p_{2} ! \cdots p_{n} !$ and

$$
t^{P}=t_{1}^{p_{1}} t_{2}^{p_{2}} \cdots t_{n}^{p_{n}}, \quad y^{M}=y_{1}^{m_{1}} \cdots y_{r}^{m_{r}}, \quad u^{N}=u_{1}^{n_{1}} \cdots u_{p}^{n_{p}} .
$$

Then it follows from the above remarks that

$$
\begin{aligned}
d\left(\mathfrak{D}^{*}\right) \chi\left(\mathfrak{D}^{*}, t, v^{*}\right) e^{-y\left(\Gamma\left(x_{t}-1, v^{*}\right)\right)} e^{-u\left(H\left(x_{t}-1, v^{*}\right)\right)} e^{-2 \rho\left(H\left(x_{t}-1, v^{*}\right)\right)} & \\
& =\sum_{M, N, P} \zeta\left(M, N, P, v^{*}\right) y^{M} u^{N} \frac{t^{P}}{P !} \quad(|t| \leqq \epsilon)
\end{aligned}
$$

where $\left(M, N, P, v^{*}\right)$ are analytic functions on $K^{*}$. $\left(d\left(\mathfrak{D}^{*}\right)\right.$ is the degree of any representation in $\mathfrak{D}^{*}$.) The above equality is to be understood in the following sense. Firstly the coefficient of $y^{M} u^{N}$ on the right-hand side is a power series in $(t)$ which converges uniformly for $|t| \leqq \epsilon$ and $v^{*} \in K^{*}$ to the coefficient of $y^{M} u^{N}$ on the left. Secondly if $\Lambda$ and $\mu$ are linear functions on $\mathfrak{h}_{p}$ and $\mathfrak{c}$ respectively, then the series obtained after the substitution $y \rightarrow \mu, u \rightarrow \Lambda$ converges uniformly to

$$
d\left(\mathfrak{D}^{*}\right) \chi\left(\mathfrak{D}^{*}, t, v^{*}\right) e^{-\mu\left(\Gamma\left(x_{t} t^{-1}, v^{*}\right)\right)} e^{-\Lambda\left(H\left(x_{t}-1, v^{*}\right)\right)} e^{-2 \rho\left(H\left(x_{t}-1, v^{*}\right)\right)}
$$

for all $|t| \leqq \epsilon$ and $v^{*} \in K^{*}$. Notice that $H\left(x^{-1}, v^{*}\right)=\Gamma\left(x^{-1}, v^{*}\right)=0$ if $x=1$. Hence the constant terms in the power series for $f_{i}\left(t, v^{*}\right)$ and $g_{j}\left(t, v^{*}\right)$ are all zero. From this it follows immediately that for a fixed $P$ there are only a finite number of values of $M$ and $N$ such that $\zeta\left(M, N, P, v^{*}\right) \neq 0$. Therefore the expression

$$
\zeta_{u, y}\left(P, v^{*}\right)=\sum_{M, N} \zeta\left(M, N, P, v^{*}\right) y^{M} u^{N}
$$

is a polynomial in $(u, y)$.

Let $\mathfrak{R}\left(\mathfrak{D}^{*}\right)$ denote the kernel (in $\mathfrak{X}$ ) of any representation of $\mathfrak{X}$ lying in $\mathfrak{D}^{*}$. Let $z \rightarrow \bar{z}$ denote the natural mapping and $\sigma$ the natural representation of $\mathfrak{X}$ on $\mathfrak{X} / \mathfrak{N}\left(\mathfrak{D}^{*}\right)=\overline{\mathfrak{X}}$. Since $\mathfrak{D}^{*} \in \Omega^{*}$ and $\operatorname{dim} \overline{\mathfrak{X}}<\infty$ we can "extend" $\sigma$ to a representation of $K^{*}$ on $\overline{\mathfrak{X}}$. Put

$$
\begin{aligned}
\eta_{u, y}^{\mathcal{D}^{*}}(P) & =\int_{K^{*}} \zeta_{u, y}\left(P, v^{*}\right) \sigma\left(v^{*}\right) \overline{1}_{d v^{*}} \\
& =\sum_{M, N} y^{M} u^{N} \int_{K^{*}} \zeta\left(M, N, P, v^{*}\right) \sigma\left(v^{*}\right) \overline{1} d v^{*}
\end{aligned}
$$


where $d v^{*}$ is the Haar measure on $K^{*}$ normalised so as to make the total measure of $K^{*}$ equal to 1 . Then $\eta_{u, v}^{\mathfrak{D}^{*}}(P) \in \overline{\mathfrak{X}}[u, y]$. Let $W_{i}$ denote the element $Z_{i} \in \mathfrak{g}$ regarded as an element of $S(\mathfrak{g})(1 \leqq i \leqq n)$. Consider the monomial $W(P)=W_{1}^{p_{1}} W_{2}^{p_{2}} \cdots W_{n}^{p_{n}}\left(p_{i}=P\left(Z_{i}\right), 1 \leqq i \leqq n\right)$ in $S(\mathfrak{g})$. Put $Z(P)=\lambda(W(P))$ where $\lambda$ is the canonical mapping of $S(\mathfrak{g})$ onto $\mathscr{B}$ (see $\S 2$ of [5]). We know that the elements $Z(P)$ form a base for $\mathfrak{B}$. Hence we can define a $(u, y)$ linear mapping $\eta_{u, y}^{\mathfrak{D}^{*}}$ of $\mathfrak{B}[u, y]$ into $\overline{\mathfrak{X}}[u, y]$ by setting $\eta_{u, y}^{\mathbb{D}^{*}}(Z(P))=\eta_{u, y}^{\mathbb{D}^{*}}(P)$. If $\Lambda$ and $\mu$ are linear functions on $\mathfrak{h}_{\mathfrak{p}}$ and $\mathfrak{c}$ respectively and $b$ is an element of $\mathfrak{B}$, we shall denote by $\eta_{\Lambda, \mu}^{\mathfrak{D}^{*}}(b)$ the value of $\eta_{u, v}^{\mathfrak{D}^{*}}(b)$ obtained under the substitution $u \rightarrow \Lambda, y \rightarrow \mu$. Obviously $\eta_{\Lambda, \mu}^{\mathbb{D}^{*}}$ is a linear mapping of $\mathscr{B}$ into $\overline{\mathfrak{X}}$. We shall use a similar notation in other cases as well, without further comment.

In the next few lemmas we study in detail the properties of the mapping $b \rightarrow \eta_{u, y}^{\mathscr{D}^{*}}(b)(b \in \mathfrak{B})$.

Put $\mathfrak{R}_{y}\left(\mathfrak{D}^{*}\right)=\beta_{-y}\left(\mathfrak{R}\left(\mathfrak{D}^{*}\right)[y]\right)$ and let $A_{y}\left(\mathfrak{D}^{*}\right)$ denote the set of all elements $a \in \mathfrak{B}[y]$ such that $\mathfrak{N}_{y}\left(\mathfrak{D}^{*}\right) a \subset \mathfrak{B} \mathfrak{N}_{y}\left(\mathfrak{D}^{*}\right)$. Clearly $A_{y}\left(\mathfrak{D}^{*}\right)$ is a subalgebra of $\mathfrak{B}[y]$ and $A_{y}\left(\mathfrak{D}^{*}\right) \supset \mathfrak{Q X}$.

LeMma 5. Let $\Re_{2}$ be the set of all elements $b \in A_{\nu}\left(\mathfrak{D}^{*}\right)[u]$ such that $\eta_{u, \nu}^{\mathfrak{D}^{*}}(b)=0$. Then $\Re_{2}$ is a left ideal in $A_{y}\left(\mathfrak{D}^{*}\right)[u]$.

Put $A_{2}=A_{y}\left(\mathfrak{D}^{*}\right)[u]$. Clearly $\Re_{2}$ is a linear subspace of $A_{2}$. We have to show that if $a(u, y) \in A_{2}$ and $b(u, y) \in \Re_{2}$ then $a(u, y) b(u, y) \in \Re_{2}$ (we write $a(u, y)$ and $b(u, y)$ to emphasise the fact that they are polynomials in $(u)$ and $(y)$ with coefficients in $\mathfrak{B})$. Suppose this is false. Then $\eta_{u, y}(a(u, y) b(u, y))$ $\neq 0$. (We write $\eta_{u, y}$ instead of $\eta_{u, y}^{\mathbb{D}^{*}}$ for convenience.) Hence we can choose linear functions $\nu$ and $\mu$ on $\mathfrak{h}_{\mathfrak{p}}$ and $\mathfrak{c}$ respectively such that the value of $\eta_{u, y}(a(u, y) b(u, y))$ obtained by the substitution $u \rightarrow \nu$ and $y \rightarrow \mu$ is not zero. Let $a_{0}, b_{0}$ denote the values of $a(u, y)$ and $b(u, y)$ under this substitution. Then $\eta_{\nu, \mu}\left(a_{0} b_{0}\right) \neq 0$. Since $\overline{\mathfrak{X}}$ is a simple algebra, there exists a maximal left ideal $\overline{\mathfrak{Y}}$ in $\overline{\mathfrak{X}}$ such that $\eta_{\nu, \mu}\left(a_{0} b_{0}\right) \in \overline{\mathfrak{Y}}$. Let $\mathfrak{Y}$ be the complete inverse image of $\overline{\mathfrak{Y}}$ in $\mathfrak{X}$. Then it is clear that the natural representation of $\mathfrak{X}$ on $\mathfrak{X} / \mathfrak{Y} \cong \overline{\mathfrak{X}} / \overline{\mathfrak{Y}}$ lies in $\mathfrak{D}^{*}$.

Let $L_{2}\left(K^{*}\right)=\mathfrak{S}$ be the Hilbert space of all square-integrable functions on $K^{*}$. We define (see $[5, \S 12]$ ) a representation $\pi_{\nu, p}$ of $G$ on $\mathfrak{S}$ as follows:

$$
\left(\pi_{\nu, \mu}(x) f\right)\left(v^{*}\right)=e^{-\mu\left(\Gamma\left(x^{-1}, v^{*}\right)\right)} e^{-(\nu+2 \rho)\left(H\left(x^{-1}, v^{*}\right)\right)} f\left(v_{x}^{*}\right)
$$

where $x \in G, v^{*} \in K^{*}$, and $f \in \mathfrak{S}$. Put

$$
\psi\left(v^{*}\right)=d\left(\mathfrak{D}^{*}\right) \chi_{\mathfrak{D}^{*}}\left(v^{*-1}\right)
$$

and let $\tau$ be the left-regular representation of $K^{*}$ on $L_{2}\left(K^{*}\right)$. Put

$$
E=d\left(\mathfrak{D}^{*}\right) \int_{K^{*}} \chi \mathscr{D}^{*}\left(v^{*-1}\right) \tau\left(v^{*}\right) d v^{*}=\int_{K^{*}} \psi\left(v^{*}\right) \tau\left(v^{*}\right) d v^{*}
$$

Then $E$ is the orthogonal projection of $\mathfrak{S}$ on the space $\mathfrak{E}_{\mathfrak{D}^{*}}$ consisting of all 
those elements in $\mathfrak{E}$ which transform under $\tau$ according to $\mathfrak{D}^{*}$. Put $\pi=\pi_{p, \mu}$ and consider $E \pi(x) \psi$. $T$ being any linear operator on $\mathfrak{S}$ we denote by $T f\left(v^{*}\right)$ the value of $T f$ at $v^{*}\left(v^{*} \in K^{*}\right)$ whenever $f$ lies in the domain of $T$. Then

$$
\begin{aligned}
E_{\pi}(x) \psi\left(v^{*}\right) & =\int_{K^{*}} \psi\left(u^{*}\right) \pi(x) \psi\left(u^{*-1} v^{*}\right) d u^{*} \\
& =\int_{K^{*}} \psi\left(v^{*} u^{*-1}\right) \pi(x) \psi\left(u^{*}\right) d u^{*}=\int_{K^{*}} \psi\left(u^{*-1} v^{*}\right) \pi(x) \psi\left(u^{*}\right) d u^{*}
\end{aligned}
$$

since $\psi\left(v^{*} u^{*-1}\right)=\psi\left(u^{*-1} v^{*}\right)$. Hence

$$
E \pi(x) \psi\left(v^{*}\right)=\int_{K^{*}} e^{\left.-\mu\left(\Gamma^{(x-1}, u^{*}\right)\right)} e^{-(\nu+2) \rho\left(H\left(x^{-1}, u^{*}\right)\right)} \psi\left(u^{*} x^{-1}\right) \tau\left(u^{*}\right) \psi\left(v^{*}\right) d u^{*}
$$

or

$$
E \pi(x) \psi=\int_{K^{*}} e^{-\mu\left(\Gamma\left(x^{-1}, v^{*}\right)\right)} e^{-(\nu+2 \rho)\left(H\left(x^{-1}, v^{*}\right)\right)} \psi\left(v_{x^{-1}}^{*}\right) \tau\left(v^{*}\right) \psi d v^{*} .
$$

Let $U$ be the linear space spanned by $\tau\left(v^{*}\right) \psi$ for all $v^{*} \in K^{*}$. Then $U=\mathfrak{S}_{\mathfrak{D}^{*}}$ and $\operatorname{dim} U=\left(d\left(\mathfrak{D}^{*}\right)\right)^{2}$. We get a representation of $K^{*}$ on $U$ which we again denote by $\tau$. $\tau$ may therefore also be regarded as a representation of $\mathfrak{t}$ (or $\mathfrak{X}$ ) on $U$. Now it is clear that $\tau(z) f=0$ if $z \in \mathfrak{N}\left(\mathfrak{D}^{*}\right)$ and $f \in U$. Hence we get a representation $\bar{\tau}$ of $\overline{\mathfrak{X}}$ on $U$ if we put $\bar{\tau}(\bar{z}) f=\tau(z) f(z \in \mathfrak{X}, f \in U), z \rightarrow \bar{z}$ being the natural mapping of $\mathfrak{X}$ on $\overline{\mathfrak{X}}$. Let $\tau^{\prime}$ be the right regular representation of $G$ on $\mathfrak{S}$. Since $\psi\left(u^{*-1} v^{*}\right)=\psi\left(v^{*} u^{*-1}\right)$ it follows that $\tau\left(u^{*}\right) \psi=\tau^{\prime}\left(u^{*-1}\right) \psi$. Hence

$$
\bar{\tau}(\bar{z}) \tau\left(u^{*}\right) \psi=\bar{\tau}(\bar{z}) \tau^{\prime}\left(u^{*-1}\right) \psi=\tau^{\prime}\left(u^{*-1}\right) \bar{\tau}(\bar{z}) \psi \quad\left(\bar{z} \in \overline{\mathfrak{X}}, u^{*} \in K^{*}\right)
$$

since right translations commute with the left translations. Now suppose $\bar{\tau}(\bar{z}) \psi=0$. Then it follows that $\bar{\tau}(\bar{z}) \tau\left(u^{*}\right) \psi=0$ for all $u^{*} \in K^{*}$. Therefore $\bar{\tau}(\bar{z})=0$ which implies that $\bar{z}=0$. Thus $\bar{\tau}(\bar{z}) \psi=0$ if and only if $\bar{z}=0$.

Let $\zeta_{\nu, \mu}\left(P, v^{*}\right)$ denote the value of the polynomial $\zeta_{u, y}\left(P, v^{*}\right)$ under the substitution $u \rightarrow \nu, y \rightarrow \mu$. Then we have seen that the series $\sum_{P} \zeta_{\nu, \mu}\left(P, v^{*}\right) t^{P} / P$ ! converges uniformly to

$$
\psi\left(v_{x_{t}^{-1}}^{*} e^{-\mu\left(\Gamma\left(x_{t}^{-1}, v^{*}\right)\right)} e^{-(v+2 \rho)\left(H\left(x_{t}-1, v^{*}\right)\right)} \quad \text { if }|t| \leqq \epsilon \text { and } v^{*} \in K^{*} .\right.
$$

Hence

$$
E \pi\left(x_{i}\right) \psi=\sum_{P} \frac{t^{P}}{P !} \int_{K^{*}} \zeta_{\nu, \mu}\left(P, u^{*}\right) \tau\left(u^{*}\right) \psi d u^{*} \quad(|t| \leqq \epsilon) .
$$

But it is clear from the definition of $\eta_{\nu, \mu}(Z(P))$ that

$$
\bar{\tau}\left(\eta_{\nu, \mu}(Z(P)) \psi=\int_{\boldsymbol{K}^{*}} \zeta_{\nu, \mu}\left(P, u^{*}\right) \tau\left(u^{*}\right) \psi d u^{*}\right.
$$


Hence

$$
E \pi\left(x_{t}\right) \psi=\sum_{P} \frac{t^{P}}{P !} \bar{\tau}\left(\eta_{\nu, \mu}(Z(P)) \psi \quad(|t| \leqq \epsilon) .\right.
$$

But we know that $\psi$ is well-behaved under $\pi(G)$ (see Lemma 34 of [5]). Hence if $|t|$ is sufficiently small

$$
\pi\left(x_{t}\right) \psi=\sum_{P} \frac{t^{P}}{P !} \pi(Z(P)) \psi
$$

where $w \rightarrow \pi(w)(w \in \mathfrak{B})$ denotes the representation of $\mathfrak{B}$ induced on the space of all well-behaved elements in $\pi(G)$. The series above is convergent in $\$$ (see $[5, \S 4])$. Since $E$ is a bounded linear operator on $\mathfrak{W}$ we get

$$
E \pi\left(x_{t}\right) \psi=\sum_{P} \frac{t^{P}}{P !} \times E \pi(Z(P)) \psi
$$

Therefore

$$
\sum_{P} \frac{t^{P}}{P !} E \pi(Z(P)) \psi=\sum_{P} \frac{t^{P}}{P !} \bar{\tau}\left(\eta_{\nu, \mu}(Z(P)) \psi\right.
$$

if $|t|$ is sufficiently small. Hence comparing coefficients of $t^{P}$ on both sides we conclude that

$$
\bar{\tau}\left(\eta_{\nu, \mu}(Z(P)) \psi=E \pi(Z(P)) \psi\right.
$$

or

$$
\bar{\tau}\left(\eta_{\nu, \mu}(a)\right) \psi=E \pi(a) \psi
$$

Now $b(u, y) \in \Re_{2}$ and therefore $\eta_{u, y}(b(u, y))=0$. Hence $\eta_{v, \mu}\left(b_{0}\right)=0$. Therefore $E \pi\left(b_{0}\right) \psi=\bar{\tau}\left(\eta_{\nu, \mu}\left(b_{0}\right)\right) \psi=0$. On the other hand

$$
E_{\pi}\left(a_{0} b_{0}\right) \psi=\bar{\tau}\left(\eta_{\nu, \mu}\left(a_{0} b_{0}\right)\right) \psi \neq 0
$$

since $\eta_{\nu, \mu}\left(a_{0} b_{0}\right) \neq 0$.

Now $b(u, y) \in A_{2}$. Hence $\beta_{-y}\left(\mathfrak{N}\left(\mathfrak{D}^{*}\right)\right) b(u, y) \subset \mathfrak{B}_{2} \beta_{-y}\left(\mathfrak{N}\left(\mathfrak{D}^{*}\right)\right)$ where $\mathfrak{B}_{2}$ $=\mathfrak{B}[u, y]$. But it is easy to verify that $\pi(u)=e^{\mu(\Gamma(u))} \tau\left(u^{*}\right)(u \in K)$ where $\Gamma(u)$ is the unique element in $c_{0}$ such that $u \exp (-\Gamma(u))$ belongs to the analytic subgroup $K^{\prime}$ of $G$ corresponding to $\mathfrak{l}_{0}^{\prime}=\left[\mathfrak{l}_{0}, \mathfrak{l}_{0}\right]$. From this it follows immediately that there exists a class $\mathfrak{D} \in \Omega$ such that $\beta_{-\mu}\left(\mathfrak{N}\left(\mathfrak{D}^{*}\right)\right)$ $=\mathfrak{N}(\mathfrak{D})(\mathfrak{N}(\mathfrak{D})$ being the kernel in $\mathfrak{X}$ of any representation of $\mathfrak{X}$ which lies in $\mathfrak{D})$, and $U$ is exactly the set of all elements in $\mathfrak{W}$ which transform under $\pi(K)$ according to $\mathfrak{D}$. Then $\mathfrak{N}(\mathfrak{D}) b_{0} \subset \mathfrak{B} \mathfrak{N}(\mathfrak{D})$. Hence $\pi\left(b_{0}\right) \psi \in U$ and therefore $\pi\left(b_{0}\right) \psi=E \pi\left(b_{0}\right) \psi=0$. Hence

$$
E \pi\left(a_{0} b_{0}\right) \psi=E \pi\left(a_{0}\right) \pi\left(b_{0}\right) \psi=0 .
$$


As this contradicts the result above the lemma is proved.

We extend the natural mapping $z \rightarrow \bar{z}$ of $\mathfrak{X}$ on $\overline{\mathfrak{X}}$ to a $(u, y)$-linear mapping of $\mathfrak{X}[u, y]$ on $\overline{\mathfrak{X}}[u, y]$. Since $\overline{\mathfrak{X}}$ is an algebra the same is true of $\overline{\mathfrak{X}}[u, y]$.

Lemma 6. If $z \in \mathfrak{X}$ and $b \in \mathfrak{B}$ then $\eta_{u, y}^{\mathfrak{D}^{*}}(z b)=\overline{\beta_{y}(z)} \eta_{u, y}^{\mathfrak{D}^{*}}(b)$. Moreover $\eta_{u, y}^{\mathfrak{D}^{*}}(1)=1$.

Again we write $\eta_{u, y}$ instead of $\eta_{u, v}^{\mathbb{D}^{*}}$. Suppose $\eta_{u, y}(z b)-\overline{\beta_{y}(z)} \eta_{u, y}(b) \neq 0$. Then we choose $\nu, \mu$ such that $\eta_{\nu, \mu}(z b)-\overline{\beta_{\mu}(z)} \eta_{\nu, \mu}(b) \neq 0$. Hence in the above notation we have

$$
E \pi(z b) \psi=\bar{\tau}\left(\eta_{v, \mu}(z b)\right) \psi .
$$

It is obvious that $E$ commutes with $\pi(u)(u \in K)$ and therefore

$$
E \pi(z b) \psi=\pi(z) E \pi(b) \psi=\pi(z) \bar{\tau}\left(\eta_{\nu, \mu}(b)\right) \psi .
$$

Moreover since $\pi(u)=e^{\mu(\Gamma(u))} \tau\left(u^{*}\right) \quad(u \in K)$ it follows that $\pi(X) \phi=\tau(X) \phi$ $\left(X \in \mathfrak{l}^{\prime}\right)$ and $\pi(\Gamma) \phi=\mu(\Gamma) \phi+\tau(\Gamma) \phi(\Gamma \in \mathfrak{c})$ for any $\phi \in U$. Hence $\pi(z) \phi=$ $\tau\left(\beta_{\mu}(z)\right) \phi(z \in \mathfrak{X}, \phi \in U)$. Therefore

$$
E \pi(z b) \psi=\tau\left(\beta_{\mu}(z)\right) \bar{\tau}\left(\eta_{\nu, \mu}(b)\right) \psi=\bar{\tau}\left(\overline{\beta_{\mu}(z)} \eta_{\nu, \mu}(b)\right) \psi .
$$

This shows that

$$
\bar{\tau}\left(\eta_{\nu, \mu}(z b)-\overline{\beta_{\mu}(z)} \eta_{\nu, \mu}(b)\right) \psi=0
$$

and therefore $\eta_{\nu, \mu}(z b)-\overline{\beta_{\mu}(z)} \eta_{\nu, \mu}(b)=0$ which contradicts our choice of $\nu, \mu$. Hence $\eta_{u, y}(z b)=\beta_{y}(z) \eta_{u, y}(b)$ and similarly we prove that $\eta_{u, y}(1)=\overline{1}$.

We use the notation of $\$ 2$ of [5] and define $\mathfrak{m}=\mathfrak{h}_{\mathfrak{f}}+\sum_{\alpha \in P_{-}} C X_{\alpha}$ $+\sum_{\alpha \in P_{-}} C X_{-\alpha}$. Then $\mathfrak{m}$ is the centraliser of $\mathfrak{h}_{\mathfrak{p}}$ in $\mathfrak{t}$.

Lemma 7. Let $\mathfrak{U}$ be the subalgebra of $\mathfrak{B}$ generated by $(1, \mathfrak{m})$. Then if $b \in \mathfrak{B}$ and $z \in \mathfrak{U}$,

$$
\boldsymbol{D}_{u, y}^{\mathfrak{D}^{*}}(b z)={\mathfrak{\mathfrak { D } ^ { * }}}_{u, y}(b) \overline{\beta_{y}(z)} \text {. }
$$

For otherwise again choose $\nu, \mu$ such that $\eta_{v, \mu}(b z)-\eta_{\nu, \mu}(b) \overline{\beta_{\mu}(z)} \neq 0$. Then we have seen above that

$$
E \pi(x) \psi=\int_{K^{*}} e^{-\mu\left(\Gamma\left(x^{-1}, u^{*}\right)\right)} e^{-(\nu+2 \rho)\left(H\left(x^{-1}, u^{*}\right)\right.} \psi\left(u_{x^{-1}}^{*}\right) \tau\left(u^{*}\right) \psi d u^{*}
$$

in the notation used above. Let $M$ be the analytic subgroup of $K$ corresponding to $\mathfrak{m}_{0}=\mathfrak{m} \cap \mathfrak{p}_{0}$. Then if $m \in K$ we know (see Lemma 37 of [5]) that $\pi(x)$ commutes with $\tau^{\prime}\left(m^{*}\right)$ where $\tau^{\prime}$ is the right regular representation of $K^{*}$ on $L_{2}\left(K^{*}\right)$. Hence

$$
\begin{aligned}
E \pi(x) \tau^{\prime}\left(m^{*-1}\right) \psi & =\tau^{\prime}\left(m^{*-1}\right) E \pi(x) \psi \\
& =\int_{K^{*}} e^{-\mu\left(\Gamma\left(x^{-1}, v^{*}\right)\right)} e^{-(\nu+2 \rho)\left(H\left(x^{-1}, v^{*}\right)\right)} \psi\left(v_{x^{-1}}^{*}\right) \tau^{\prime}\left(m^{*-1}\right) \tau\left(v^{*}\right) \psi d v^{*} .
\end{aligned}
$$


But we have seen above that $\tau^{\prime}\left(m^{*-1}\right) \psi=\tau\left(m^{*}\right) \psi$ and therefore $\tau^{\prime}\left(m^{*-1}\right)$ $\cdot \tau\left(v^{*}\right) \psi=\tau\left(v^{*} m^{*}\right) \psi$. Therefore

$$
E \pi(x) \tau\left(m^{*}\right) \psi=\int_{K^{*}} e^{-\mu\left(\Gamma\left(x^{-1}, v^{*}\right)\right)} e^{-(\nu+2 \rho)\left(H\left(x^{-1}, v^{*}\right)\right)} \psi\left(v_{x^{-1}}^{*}\right) \tau\left(v^{*} m^{*}\right) \psi d v^{*} .
$$

From this it follows immediately that

$$
E \pi\left(b^{\prime}\right) \tau\left(z^{\prime}\right) \psi=\bar{\tau}\left(\eta_{\nu, \mu}\left(b^{\prime}\right)\right) \tau\left(z^{\prime}\right) \psi \quad\left(b^{\prime} \in \mathfrak{B}, z^{\prime} \in \mathfrak{U}\right) .
$$

But we have seen above that $\pi\left(z^{\prime}\right) \psi=\tau\left(\beta_{\mu}\left(z^{\prime}\right)\right) \psi\left(z^{\prime} \in \mathfrak{X}\right)$. Therefore $E \pi(b z) \psi$ $=E \pi(b) \tau\left(\beta_{\mu}(z)\right) \psi$. Let $\mathfrak{U}^{\prime}$ be the set of all elements $w \in \mathfrak{X}$ such that $\beta_{\mu}(w) \in \mathfrak{U}$. Since $w \rightarrow \beta_{\mu}(w)(w \in \mathfrak{X})$ is a homomorphism it follows that $\mathfrak{U}^{\prime}$ is a subalgebra of $\mathfrak{X}$. Moreover $\mathfrak{U}^{\prime} \supset \mathfrak{m}$ and therefore $\mathfrak{U}^{\prime} \supset \mathfrak{U}$. Hence $\beta_{\mu}(z) \in \mathfrak{U}$ and therefore

$$
\begin{aligned}
E \pi(b z) \psi & =\bar{\tau}\left(\eta_{\nu, \mu}(b)\right) \tau\left(\beta_{\mu}(z)\right) \psi \\
& =\bar{\tau}\left(\eta_{\nu, \mu}(b) \overline{\beta_{\mu}(z)}\right) \psi .
\end{aligned}
$$

On the other hand $E \pi(b z) \psi=\bar{\tau}\left(\eta_{\nu, \mu}(b z)\right) \psi$ and so we conclude that

$$
\bar{\tau}\left(\eta_{\nu, \mu}(b z)-\eta_{\nu, \mu}(b) \overline{\beta_{\mu}(z)}\right) \psi=0
$$

which contradicts the fact that $\eta_{\nu, \mu}(b z)-\eta_{\nu, \mu}(b) \overline{\beta_{u}(z)} \neq 0$. Thus the lemma is proved.

We define the automorphism $\theta$ of $g$ and the sets $P, P_{+}$, and $P_{-}$of positive roots of $\mathfrak{g}$ (with respect to $\mathfrak{h}$ ) as in $\$ 2$ of [5]. Let $\alpha_{1}<\alpha_{2}<\cdots<\alpha_{r}$ be all the roots in $P$. We recall that if $\alpha \in P_{-}$and $\alpha^{\prime} \in P_{+}$then $\alpha^{\prime}>\alpha$. Suppose then that $\alpha_{1}, \cdots, \alpha_{s} \in P_{-}$and $\alpha_{s+1}, \cdots, \alpha_{r} \in P_{+}(0 \leqq s \leqq r)$. First we make the following observation.

Lemma 8. Let $p_{i}, q_{j}$ be non-negative integers such that $p_{1} \alpha_{1}+\cdots+p_{r} \alpha_{r}$ $=q_{1} \alpha_{1}+\cdots+q_{8} \alpha_{8}$. Then $p_{s+1}=p_{s+2}=\cdots=p_{r}=0$.

For $p_{8+1} \alpha_{8+1}+\cdots+p_{r} \alpha_{r}=\left(q_{1}-p_{1}\right) \alpha_{1}+\cdots+\left(q_{s}-p_{8}\right) \alpha_{8}$. Applying $\theta$ to both sides we get

$$
p_{s+1} \theta \alpha_{s+1}+\cdots+p_{r} \theta \alpha_{r}=\left(q_{1}-p_{1}\right) \alpha_{1}+\cdots+\left(q_{s}-p_{s}\right) \alpha_{s}
$$

since $\theta \alpha=\alpha$ for $\alpha \in P_{-}$. Therefore

$$
p_{s+1}\left(\alpha_{s+1}-\theta \alpha_{s+1}\right)+p_{s+2}\left(\alpha_{s+2}-\theta \alpha_{s+2}\right)+\cdots+p_{r}\left(\alpha_{r}-\theta \alpha_{r}\right)=0 .
$$

But $\theta \alpha<0$ for $\alpha \in P_{+}$and therefore $\alpha_{t}-\theta \alpha_{t}>0$ for $t>s$. Hence the above relation is possible only if $p_{s+1}=\cdots=p_{r}=0$.

Let $\Lambda$ be a linear function on $\mathfrak{h}$. We say that an element $b \in \mathfrak{B}$ is of rank $\Lambda$ if $[H, b]=\Lambda(H) b$ for all $H \in \mathfrak{h}$.

LemMa 9. Let $\mathfrak{B}$ be the subalgebra of $\mathfrak{B}$ generated by $\left(1, \mathfrak{m}+\mathfrak{h}_{\mathfrak{p}}\right)$. Then for any $z \in \mathfrak{Z}$ there exists an element $z_{0} \in \mathfrak{B}$ such that 


$$
z-z_{0} \in \sum_{\alpha, \beta \in P+} X_{-\alpha} \mathfrak{B} X_{\beta}
$$

and $z_{0}$ is of the rank zero.

We use the notation of $[5, \S 2]$ and the lemma above. Choose a base $H_{p+1}, \cdots, H_{l}$ for $\mathfrak{h}_{\mathfrak{l} .}$ Then $X_{-\alpha_{r}}, \cdots, X_{-\alpha_{1}}, H_{1}, \cdots, H_{l}, X_{\alpha_{1}}, \cdots, X_{\alpha_{r}}$ is a base for $\mathfrak{g}$. Hence $z$ can be written uniquely (see [2]) in the form

$$
z=\sum_{(p),(m),(q)} a((p),(m),(q)) X_{-\alpha_{r}}^{p r} \cdots X_{-\alpha_{1}}^{p_{1}} H_{1}^{m_{1}} \cdots H_{l}^{m l} X_{\alpha_{1}}^{q_{1}} \cdots X_{\alpha_{r}}^{q_{r}}
$$

where $a((p),(m),(q)) \in C$. Since $z \in \mathbb{Z}, z$ is of rank zero and therefore it follows (see [3, proof of Lemma 36]) that $a((p),(m),(q))=0$ unless $p_{1} \alpha_{1}+\cdots$ $+p_{r} \alpha_{r}=q_{1} \alpha_{1}+\cdots+q_{r} \alpha_{r}$. Now

$$
X_{-\alpha_{r}}^{p_{r}} \cdots X_{-\alpha_{1}}^{p_{1}} H_{1}^{m_{1}} \cdots H_{l}^{m l} X_{\alpha_{1}}^{q_{1}} \cdots X_{\alpha_{r}}^{q_{r}} \in \sum_{\alpha, \beta \in P_{+}} X_{-\alpha} \mathfrak{B} X_{\beta}
$$

unless either $p_{s+1}=\cdots=p_{r}=0$ or $q_{s+1}=\cdots=q_{r}=0$. But if $a((p),(m),(q))$ $\neq 0$ we conclude from Lemma 8 that $p_{s+1}=\cdots=p_{r}=0$ if and only if $q_{s+1}=\cdots$ $=q_{r}=0$. Hence $z \equiv z_{0} \bmod \sum_{\alpha, \beta \in P_{+}} X_{-\alpha} \mathfrak{B} X_{\beta}$ where

$$
z_{0}=\sum a((p),(m),(q)) X_{-\alpha_{s}}^{p_{s}} \cdots X_{-\alpha_{1}}^{p_{1}} H_{1}^{m_{1}} \cdots H_{l}^{m l} X_{\alpha_{1}}^{q_{1}} \cdots X_{\alpha_{s}}^{q_{s}} \in \mathfrak{B},
$$

the sum extending only to those terms for which $p_{j}=q_{j}=0(s<j \leqq r)$. Since $z_{0}$ is of rank zero the lemma is proved.

Since $\mathfrak{g}$ is the direct sum of $\mathfrak{l}, \mathfrak{h}_{\mathfrak{p}}$, and $\mathfrak{n}=\sum_{\alpha \in P_{+}} C X_{\alpha}$ it follows (see [2]) that for any $b \in \mathfrak{B}$ there exist unique elements $x\left(m_{1}, \cdots, m_{p}\right)$ in $\mathfrak{X}$ such that

$$
b \equiv \sum_{(m)} H_{1}^{m_{1}} \cdots H_{p}^{m_{p}} x\left(m_{1}, \cdots, m_{p}\right) \bmod \sum_{\alpha \in P_{+}} X_{\alpha} \mathfrak{B} .
$$

We now define a linear mapping $\xi_{u}$ of $\mathfrak{B}$ into $\mathfrak{X}[u]$ by setting

$$
\xi_{u}(b)=\sum_{(m)} u_{1}^{\prime m_{1}} \cdots u_{p}^{\prime m_{p}} x\left(m_{1}, \cdots, m_{p}\right)
$$

where $u_{i}^{\prime}=u_{i}+2 \rho\left(H_{i}\right), 1 \leqq i \leqq p$. As before let $\mathfrak{Q}$ denote the centraliser of $\mathfrak{X}$ in $\mathfrak{B}$.

LemmA 10. If $a \in \mathfrak{Q}$ and $b \in \mathfrak{B}$, then $\xi_{u}(b a)=\xi_{u}(a) \xi_{u}(b)$.

For in the above notation

$$
b a \equiv \sum_{(m)} H_{1}^{m_{1}} \cdots H_{p}^{m_{p}} a x\left(m_{1}, \cdots, m_{p}\right) \bmod \sum_{\alpha \in P+} X_{\alpha} \mathfrak{B},
$$

since $a x\left(m_{1}, \cdots, m_{p}\right)=x\left(m_{1}, \cdots, m_{p}\right) a$. Hence if we observe that 


$$
H_{1}^{m_{1}} \cdots H_{p}^{m_{p}} X_{\alpha} \in X_{\alpha} \mathfrak{B}
$$

our assertion follows.

The significance of the mapping $\xi_{u}$ derives from the following result.

Lemma 11. If $a \in \mathfrak{Q}$, then $\eta_{u, v}^{\mathbb{D}^{*}}(a)=\overline{\beta_{y}\left(\xi_{u}(a)\right) \text {. }}$

For otherwise we could choose $\nu, \mu$ such that $\eta_{\nu, \mu}(a)-\overline{\beta_{\mu}\left(\xi_{\nu}(a)\right)} \neq 0$. Let $N$ be the analytic subgroup of $G$ corresponding to the subalgebra $\mathfrak{n}_{0}=\mathfrak{n} \cap \mathfrak{g}_{0}$ of go. For any $x \in G$ and $v \in K$ put $x^{v}=v x v^{-1}$ and let $x \rightarrow \operatorname{Ad}(x)$ denote the adjoint representation of $G$. Extend $\operatorname{Ad}(x)$ to an automorphism of $\mathfrak{B}$ over $C$ and put $b^{v}=\operatorname{Ad}(v) b(b \in \mathfrak{B})$. Then if $f \in L_{2}\left(K^{*}\right)$ and $\pi=\pi_{v, \mu}$ (in the notation of the proof of Lemma 5) we find that

$$
\begin{aligned}
& \pi\left(n^{v}\right) f\left(v^{*}\right)=f\left(v^{*}\right) \\
& (n \in N) \text {, } \\
& \pi\left(u^{v}\right) f\left(v^{*}\right)=e^{\mu(\Gamma(u))} f\left(v^{*} u^{*-1}\right) \\
& (u \in K) \text {, } \\
& \pi\left(h^{v}\right) f\left(v^{*}\right)=e^{(v+2 \rho)(\log h)} f\left(v^{*}\right) \\
& \left(h \in A_{+}\right) \text {. }
\end{aligned}
$$

Here $A_{+}$is the analytic subgroup of $G$ corresponding to $\mathfrak{h}_{\mathfrak{p}_{0}}$ and $\log h\left(h \in A_{+}\right)$ denotes the unique element $H \in \mathfrak{h}_{\mathfrak{p}_{0}}$ such that $\exp H=h$. From this it follows that if $f$ is an indefinitely differentiable function on $K^{*}$ which is well-behaved under $\pi$ then

$$
\begin{aligned}
& \pi\left(X^{v}\right) f\left(v^{*}\right)=0 \\
& (X \in \mathfrak{n}), \\
& \pi\left(H^{v}\right) f\left(v^{*}\right)=(\nu(H)+2 \rho(H)) f\left(v^{*}\right) \\
& \left(H \in \mathfrak{h}_{\mathfrak{p}}\right) \text {. }
\end{aligned}
$$

Now let us consider $\pi(a) \psi$ where $\psi$ is the function introduced in the proof of Lemma 5. Let $v$ be a fixed element in $K$. Since $a \in \mathfrak{Q}, a=a^{v}$. Let $a \equiv a_{0} \bmod$ $\sum_{\alpha \in P_{+}} X_{\alpha} \mathfrak{B}$ where

$$
a_{0}=\sum_{(m)} H_{1}^{m_{1}} \cdots H_{p}^{m_{p}} z\left(m_{1}, \cdots, m_{p}\right) \quad\left(z\left(m_{1}, \cdots, m_{p}\right) \in \mathfrak{X}\right) .
$$

Then $a^{v}-a_{0}^{0} \in \sum_{\alpha \in P_{+}} X_{\alpha}^{0} \mathfrak{B}$ and therefore

$$
\pi(a) \psi\left(v^{*}\right)=\pi\left(a^{v}\right) \psi\left(v^{*}\right)=\pi\left(a_{0}^{v}\right) \psi\left(v^{*}\right)+\sum_{\alpha \in P+} \pi\left(X_{\alpha}^{v}\right) \pi\left(b_{\alpha}\right) \psi\left(v^{*}\right),
$$

where $b_{\alpha} \in \mathfrak{B}$. But if we put $f_{\alpha}=\pi\left(b_{\alpha}\right) \psi\left(\alpha \in P_{+}\right)$we know from our discussion above that $\pi\left(X_{\alpha}^{0}\right) f_{\alpha}\left(v^{*}\right)=0$. Hence

$$
\pi(a) \psi\left(v^{*}\right)=\pi\left(a_{0}^{0}\right) \psi\left(v^{*}\right) .
$$

We find similarly that

$$
\pi\left(a_{0}^{\nabla}\right) \psi\left(v^{*}\right)=\sum_{(m)} \nu_{1}^{\prime m_{1}} \cdots \nu_{p}^{\prime m_{p}} \pi\left(\left(z\left(m_{1}, \cdots, m_{p}\right)\right)^{v}\right) \psi\left(v^{*}\right)=\pi\left(\left(\xi_{\nu}(a)\right)^{v}\right) \psi\left(v^{*}\right)
$$


where $\nu_{i}^{\prime}=\nu\left(H_{i}\right)+2 \rho\left(H_{i}\right), 1 \leqq i \leqq p$. But $\psi\left(v^{*} u^{*-1}\right)=\psi\left(u^{*-1} v^{*}\right)\left(u^{*} \in K^{*}\right)$ and therefore

$$
\pi\left(u^{v}\right) \psi\left(v^{*}\right)=e^{\mu(\Gamma(u))} \psi\left(u^{*-1} v^{*}\right)=\pi(u) \psi\left(v^{*}\right) .
$$

Hence we conclude that

$$
\pi\left(z^{v}\right) \psi\left(v^{*}\right)=\pi(z) \psi\left(v^{*}\right)
$$

and therefore

$$
\pi(a) \psi\left(v^{*}\right)=\pi\left(\left(\xi_{\nu}(a)\right)^{v}\right) \psi\left(v^{*}\right)=\pi\left(\xi_{\nu}(a)\right) \psi\left(v^{*}\right) .
$$

Since this is true for every $v^{*} \in K^{*}, \pi(a) \psi=\pi\left(\xi_{\nu}(a)\right) \psi$. Now define the projection $E$ and the representation $\bar{\tau}$ of $\overline{\mathfrak{X}}$ as in the proof of Lemma 5. Since $a \in \mathfrak{Q}$, $\pi(a) \psi=E \pi(a) \psi=\bar{\tau}\left(\eta_{\nu, \mu}(a)\right) \psi$, and

$$
\pi\left(\xi_{\nu}(a)\right) \psi=\bar{\tau} \overline{\left(\beta_{\mu}\left(\xi_{\nu}(a)\right)\right.} \psi
$$

from Lemma 6. Therefore $\bar{\tau}\left(\eta_{\nu, \mu}(a)-\overline{\left.\beta_{\mu}\left(\xi_{\nu}(a)\right)\right)} \psi=0\right.$ which implies that $\eta_{\nu, \mu}(a)-\overline{\beta_{\mu}\left(\xi_{\nu}(a)\right)}=0$. Since this contradicts our choice of $\nu, \mu$ the lemma follows.

In view of Lemmas 6 and 11 the mapping $\eta_{u, v}^{\mathfrak{D}^{*}}$ is now completely determined on $\mathfrak{A}=\mathfrak{Q} \mathfrak{X}=\mathfrak{X} \mathfrak{Q}$. If $a=\sum_{j=1}^{s} x_{j} a_{j}\left(x_{j} \in \mathfrak{X}, a_{j} \in \mathfrak{Q}\right)$ then

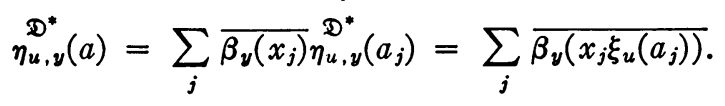

Notice that if $a=0, \overline{\beta_{y}\left(\sum_{j} x_{j} \xi_{u}\left(a_{j}\right)\right)}=0$ and therefore

$$
\beta_{y}\left(\sum_{j} x_{j} \xi_{u}\left(a_{j}\right)\right) \in \mathfrak{N}\left(\mathfrak{D}^{*}\right)[u, y] .
$$

Since this is true for every $\mathfrak{D}^{*} \in \Omega^{*}$ and since we know that $\bigcap_{\mathfrak{D}^{*} \in \Omega^{*}} \mathfrak{N}\left(\mathfrak{D}^{*}\right)$ $=\{0\}$ (see [2]) it follows that $\beta_{y}\left(\sum_{j} x_{j} \xi_{u}\left(a_{j}\right)\right)=0$. Hence the mapping $\xi_{u, y}$ : $\sum_{j} x_{j} a_{j} \rightarrow \beta_{y}\left(\sum x_{j} \xi_{u}\left(a_{j}\right)\right)\left(x_{j} \in \mathfrak{X}, a_{j} \in \mathfrak{Q}\right)$ is a well-defined linear mapping of $\mathfrak{A}$ into $\mathfrak{X}[u, y]$ and $\eta_{u, y}^{\mathfrak{D}^{*}}(a)=\overline{\xi_{u, y}(a)}(a \in \mathfrak{X})$.

Let $\mathfrak{D}_{0}^{*}$ be a class in $\Omega^{*}$. As before we denote by $A_{y}\left(\mathfrak{D}_{0}^{*}\right)$ the set of all $a \in \mathfrak{B}[y]$ such that $\beta_{-y}\left(\mathfrak{N}\left(\mathfrak{D}_{0}^{*}\right)\right) a \subset \mathfrak{B}_{1} \beta_{-y}\left(\mathfrak{N}\left(\mathfrak{D}_{0}^{*}\right)\right)$ where $\mathfrak{B}_{1}=\mathfrak{B}[y]$. Let $\mu$ be a linear function on $\left(\mathcal{C}\right.$. We define a 1-1 mapping $\mathfrak{D} \rightarrow \mathfrak{D}_{\mu}$ of $\Omega$ onto itself such that $\mathfrak{N}\left(\mathfrak{D}_{\mu}\right)=\beta_{-\mu}(\mathfrak{N}(\mathfrak{D}))(\mathfrak{N}(\mathfrak{D})$ is the kernel in $\mathfrak{X}$ of any representation in $\mathfrak{D})$. In particular put $\mathfrak{D}_{0}=\left(\mathfrak{D}_{0}^{*}\right)_{\mu}$. For any $\mathfrak{D} \in \Omega$ let $A(\mathfrak{D})$ denote the set of all elements $a \in \mathfrak{B}$ such that $\mathfrak{R}(\mathfrak{D}) a \subset \mathfrak{B} \mathfrak{R}(\mathfrak{D})$. If $a(y) \in \mathfrak{B}[y]$ we shall write $a(\mu)$ to denote the value of the polynomial under the substitution $y \rightarrow \mu$. It is obvious that if $a(y) \in A_{y}\left(\mathfrak{D}_{0}^{*}\right)$, then $a(\mu) \in A\left(\mathfrak{D}_{0}\right)$.

Lemma 12. For any $a_{0} \in A\left(\mathfrak{D}_{0}\right)$ we can find an element $a(y) \in A_{y}\left(\mathfrak{D}_{0}^{*}\right)$ such that $a_{0}=a(\mu)$.

Before proving this lemma we make some general observations. Put 
$\mathfrak{B}_{1}=\mathfrak{B}[y]$ and $\mathfrak{U}=\mathfrak{B}_{1} \beta_{-y}\left(\mathfrak{N}\left(\mathfrak{D}_{0}^{*}\right)\right)$. Let $\pi$ denote the natural representation and $b \rightarrow b^{*}$ the natural mapping of $\mathfrak{B}_{1}$ on $\mathfrak{B}_{1}^{*}=\mathfrak{B}_{1} / \mathfrak{U}$. For any $\mathfrak{D} \in \Omega$ we denote by $\mathfrak{B}_{1}^{*}(\mathfrak{D})$ the set of all $b^{*} \in \mathfrak{B}_{1}^{*}$ such that $\pi\left(\beta_{-y}(\mathfrak{N}(\mathfrak{D}))\right) b^{*}=0$. Put $\pi^{*}(z)$ $=\pi\left(\beta_{-y}(z)\right) \quad(z \in \mathfrak{X})$. Then $\pi^{*}$ is a representation of $\mathfrak{X}$ on $\mathfrak{B}_{1}^{*}$ and $\mathfrak{B}_{1}^{*}(\mathfrak{D})$ is exactly the set of those elements in $\mathfrak{B}_{1}^{*}$ which transform under $\pi^{*}$ according to $\mathfrak{D}$. We shall show that $\mathfrak{B}_{1}^{*}=\sum_{\mathfrak{D} \in \Omega} \mathfrak{B}_{1}^{*}(\mathfrak{D})$. Since $1^{*} \in \mathfrak{B}_{1}^{*}\left(\mathfrak{D}_{0}^{*}\right)$ it is sufficient to prove that $V^{*}=\sum_{\mathfrak{D} \in \Omega} \mathfrak{B}_{1}^{*}(\mathfrak{D})$ is invariant under $\pi(\mathfrak{g}[y])$. But it is obvious that $V^{*}$ is invariant under $\pi(\mathfrak{f}[y])$. Therefore it would be sufficient to show that $\pi(Y) b^{*} \in V^{*}$ for $b^{*} \in \mathfrak{B}_{1}^{*}(\mathfrak{D})(\mathfrak{D} \in \Omega)$ and $Y \in \mathfrak{p}$. Let $U=\pi^{*}(\mathfrak{X}) b^{*}$. Then $\operatorname{dim} U<\infty$ and the representation $\pi_{U}^{*}$ of $\mathfrak{X}$ induced on $U$ under $\pi^{*}$ is semisimple. Moreover if $X \in \mathfrak{f}$ and $Y \in \mathfrak{p}, X Y=[X, Y]+Y X$ and $[X, Y] \in \mathfrak{p}$. Hence

$$
\pi^{*}(X) \pi(Y) a^{*}=\pi(\sigma(X) Y) a^{*}+\pi(Y) \pi^{*}(X) a^{*}
$$$$
\left(a^{*} \in U\right)
$$

where $\sigma$ is the representation of $\mathfrak{f}$ on $\mathfrak{p}$ given by $\sigma(X) Y=[X, Y]$. Now we regard $\mathfrak{p} \times U$ as a $\mathfrak{k}$-module under the representation $\sigma+\pi_{U}^{*}$ of $\mathfrak{t}$. Consider the mapping $\phi: Y \times a^{*} \rightarrow \pi(Y) a^{*}\left(Y \in \mathfrak{p}, a^{*} \in U\right)$ of $\mathfrak{p} \times U$ onto $\pi(\mathfrak{p}) U$. Then if $X \in \mathfrak{l}$ we may write the above relation as follows:

$$
\phi\left(\sigma(X) Y \times a^{*}+Y \times \pi^{*}(X) a^{*}\right)=\pi(X) \phi\left(Y \times a^{*}\right) .
$$

This proves that the $\mathfrak{f}$-module $\pi(\mathfrak{p}) U$ is a homomorphic image of the $\mathfrak{f}$-module $\mathfrak{p} \times U$. Since $\sigma$ and $\pi_{U}^{*}$ are semisimple representations, $\mathfrak{p} \times U$ is a semisimple $\mathfrak{l}$-module (see Lemma 8 of [5]). The same therefore holds for $\pi(\mathfrak{p}) U$. Hence $\pi(\mathfrak{p}) U \subset V^{*}$ and so our assertion is proved. Thus the representation $\pi^{*}$ is quasi semisimple and the sum $\sum_{\mathscr{D} \in \Omega} \mathfrak{B}_{1}^{*}(\mathfrak{D})$ is direct (see Lemma 6 of [5]).

Now we come to the lemma. Put $\mathfrak{B}_{\mu}=\sum_{1 \leqq i \leqq r} \mathfrak{B}_{1}\left(y_{i}-\mu\left(\Gamma_{i}\right)\right)$ where $\Gamma_{1}, \cdots, \Gamma_{r}$ is the base for $c$ which we chose in $\$ 2$. Let $\bar{\pi}$ be the natural representation and $b \rightarrow \bar{b}\left(b \in \mathfrak{B}_{1}\right)$ the natural mapping of $\mathfrak{B}_{1}$ on $\mathfrak{B}_{1} /\left(\mathfrak{B}_{\mu}+\mathfrak{U}\right)$. We may clearly identify $\overline{\mathfrak{B}_{1}}$ with $\mathfrak{B}_{1}^{*} / \mathfrak{B}_{\mu}^{*}$ in the natural way. Let $\overline{\mathfrak{B}(\mathfrak{D})}$ denote the image of $\mathfrak{B}_{1}^{*}(\mathfrak{D})$ in $\mathfrak{B}_{1}^{*} / \mathfrak{B}_{\mu}^{*}=\overline{\mathfrak{B}_{1}}$. Then $\overline{\mathfrak{B}_{1}}=\sum_{\mathfrak{D} \in \Omega} \overline{\mathfrak{B}(\mathfrak{D})}$. Moreover if $a^{*} \in \mathfrak{B}_{1}^{*}(\mathfrak{D}), \pi\left(\beta_{-y}(\mathfrak{N}(\mathfrak{D})) a^{*}=\{0\}\right.$. Hence if $\overline{a^{*}}$ is the image of $a^{*}$ in $\overline{\mathfrak{B}(\mathfrak{D})}$, $\bar{\pi}\left(\beta_{-\mu}(\mathfrak{N}(\mathfrak{D}))\right) \overline{a^{*}}=\{0\}$ and therefore $\overline{a^{*}}$ transforms under $\overline{\boldsymbol{\pi}}(\mathfrak{f})$ according to $\mathfrak{D}_{\mu}$. This shows that the representation $\bar{\pi}(\mathfrak{f})$ is quasi semisimple. Now it is obvious that if $a \in \mathfrak{B}[y]$ then $a^{*} \in \mathfrak{B}_{1}^{*}\left(\mathfrak{D}_{0}^{*}\right)$ if and only if $a \in A_{y}\left(\mathfrak{D}_{0}^{*}\right)$. Similarly an element $a$ of $\mathfrak{B}$ lies in $A\left(\mathfrak{D}_{0}\right)$ if and only if $\bar{a} \in \overline{\mathcal{B}\left(\mathfrak{D}_{0}^{*}\right)}$. Now let $a_{0} \in A\left(\mathfrak{D}_{0}\right)$. Then it follows from what we have said above that we can find $a_{1}(y) \in A_{y}\left(\mathfrak{D}_{0}^{*}\right)$ such that $\bar{a}_{0}=\overline{a_{1}(y)}$. Let $a_{1}(\mu)$ be the value of $a_{1}(y)$ under the substitution $y \rightarrow \mu$. It is obvious that $\overline{a_{1}(y)}=\overline{a_{1}(\mu)}$. Hence $\overline{a_{0}-a_{1}(\mu)}=0$. Now it is easy to see that if $b \in \mathfrak{B}$ then $\bar{b}=0$ if and only if $b \in \mathfrak{B} \mathfrak{N}\left(\mathfrak{D}_{0}\right)$. Hence $a_{0}-a(\mu) \in \mathfrak{B N}\left(\mathfrak{D}_{0}\right)$ and therefore $\beta_{\mu}\left(a_{0}-a_{1}(\mu)\right) \in \mathfrak{B N}\left(\mathfrak{D}_{0}^{*}\right)$ and $\beta_{-y}\left(\beta_{\mu}\left(a_{0}-a_{1}(\mu)\right) \in \mathfrak{B}_{1} \beta_{-y}\left(\mathfrak{N}\left(\mathfrak{D}_{0}^{*}\right)\right)\right.$ $\subset A_{y}\left(\mathfrak{D}_{0}^{*}\right)$. So $a_{2}(y)=\beta_{-y}\left(\beta_{\mu}\left(a_{0}-a_{1}(\mu)\right)\right.$ is in $A_{y}\left(\mathfrak{D}_{0}^{*}\right)$ and $a_{2}(\mu)=\beta_{-\mu}\left(\beta_{\mu}\left(a_{0}\right)\right.$ $\left.-a_{1}(\mu)\right)=a_{0}-a_{1}(\mu)$. Therefore if we put $a(y)=a_{1}(y)+a_{2}(y), a(\mu)=a_{0}$ and this proves our result. 
We shall keep $\mathfrak{D}_{0}^{*}$ fixed in the rest of this discussion. Put $A_{2}=A_{y}\left(\mathfrak{D}_{0}^{*}\right)[u]$ and let $\Re_{2}$ be the set of all $a \in A_{2}$ such that $\eta_{u, y}(a)=0 \quad\left(\eta_{u, y}=\eta_{u, y} \mathbb{D}^{*}\right)$. Extend the natural mapping of $\mathfrak{X}$ on $\overline{\mathfrak{X}}=\mathfrak{X} / \mathfrak{N}\left(\mathfrak{D}_{0}^{*}\right)$ to a $(u, y)$-linear mapping $z \rightarrow \bar{z}$ of $\mathfrak{X}[u, y]$ on $\overline{\mathfrak{X}}[u, y]$. Then if $a \in A_{2}$ we can choose $w \in \mathfrak{X}[u, y]$ such that $\eta_{u, y}(a)$ $=\bar{w}$. Hence if $z=\beta_{-y}(w), \eta_{u, y}(a-z)=0$ from Lemma 6 . This shows that if $z_{1}, \cdots, z_{d}$ is a base for $\mathfrak{X} \bmod \mathfrak{N}\left(\mathfrak{D}_{0}^{*}\right)$ then $A_{2}=\Re_{2}+\sum_{1 \leqq i \leqq d} C[u, y] z_{i}^{\prime}$ where $z_{i}^{\prime}=\beta_{-y}\left(z_{i}\right), 1 \leqq i \leqq d$. Therefore if $b \in A_{y}\left(D_{0}^{*}\right)$ we can choose $c_{i j}(u, y) \in C[u, y]$ such that

$$
b z_{i}^{\prime}-\sum_{1 \leqq j \leqq d} c_{i j}(u, y) z_{j}^{\prime} \in \Re_{2} \quad(1 \leqq i \leqq r)
$$

Put

$$
f(T, u, y)=\operatorname{det}\left(T \delta_{i j}-c_{i j}(u, y)\right)_{l \leqq i, j \leqq d},
$$

where $T$ is an indeterminate. Then $f(T, u, y)$ is a polynomial of degree $d$ in $T$ with coefficients in $C[u, y]$. Let $x_{1}, \cdots, x_{l}$ be $l$ independent indeterminates $(l=\operatorname{dim} \mathfrak{h})$. Put $x\left(H_{i}\right)=x_{i}, 1 \leqq i \leqq l$, and define $x(H)(H \in \mathfrak{h})$ by linearity. Let $W$ be the Weyl group of $\mathfrak{g}$ with respect to $\mathfrak{h}$. We denote by $f^{(\sigma)}(T, x, y)$ $(\sigma \in W)$ the polynomial in $T$ (with coefficients in $C[x, y]$ ) obtained from $f(T$, $u, y)$ under the substitution $u_{i} \rightarrow x\left(\sigma^{-1} H_{i}\right)+\rho\left(\sigma^{-1} H_{i}\right)-\rho\left(H_{i}\right)(1 \leqq i \leqq p)$. Put

$$
F(T, x, y)=\prod_{\sigma \in W} f^{(\sigma)}(T, x, y) .
$$

Then if $h$ is the order of the group $W, F$ is of degree $d h$ in $T$. Let $\chi_{x}$ denote the mapping of $\mathbb{B}$ into $C[x]$ defined as follows. If $z$ $\equiv \sum_{(m)} a\left(m_{1}, \cdots, m_{l}\right) H_{1}^{m_{1}} H_{2}^{m_{2}} \cdots H_{l}^{m_{l}} \bmod \sum_{\alpha \in P} \mathfrak{B} X_{\alpha}\left(a\left(m_{1}, \cdots, m_{l}\right)\right.$ $\in C, z \in 3)$ then $\chi_{x}(z)=\sum_{(m)} a\left(m_{1}, \cdots, m_{l}\right) x_{1}^{m_{1}} \cdots x_{l}^{m_{l}}$. We have seen in $\left[3\right.$, Lemma 36] that $\chi_{x}$ is an isomorphism of $\not$ into $C[x]$. Extend $\chi_{x}$ to a (y)-linear mapping of $3[y]$ into $C[x, y]$. It follows from Lemma 38 of [3] that every coefficient of $F$ (regarded as a polynomial in $T$ ) is of the form $\chi_{x}(z(y))$ where $z(y)$ is some element in $3[y]$. Therefore

$$
F(T, x, y)=\sum_{0 \leq q \leq h d} T^{q} \chi_{x}\left(z_{q}(y)\right) \quad\left(z_{q}(y) \in B[y]\right) .
$$

For any $\mathfrak{D} \in \Omega^{*}$ let $\mathfrak{D}^{\prime}$ denote the equivalence class of the representation $\sigma^{\prime}$ of $\mathfrak{t}^{\prime}=[\mathfrak{l}, \mathfrak{t}]$ defined by any $\sigma \in \mathfrak{D}$. We denote by $\Omega_{F}$ the set of those $\mathfrak{D} \in \Omega$ for which $\mathfrak{D}^{\prime} \in \Omega_{F}^{\prime}$ (see $\S 1$ for the definition of $\Omega_{F}^{\prime}$ ). Then we have the following result which is one of the main steps of our argument.

Lemma 13. Suppose $\mathfrak{D}_{0}^{*} \in \Omega_{F}^{*}=\Omega_{F} \cap \Omega^{*}$. Then

$$
\beta_{\nu}\left(\sum_{0 \leqq q \leqq h d} b^{q} z_{q}(y)\right) \in \mathfrak{B N}\left(\mathfrak{D}_{0}^{*}\right)[y] .
$$

First we observe that if $\{\mathfrak{Y}\}$ is any collection of left ideals in $\mathfrak{X}$ then 
$\cap(\mathfrak{B V})=\mathfrak{B}(\cap \mathfrak{Y})$. For let $\lambda$ be the canonical mapping of the symmetric algebra $S(\mathfrak{g})$ onto $\mathfrak{B}$. Then if $\{Q\}$ is a base for $S(\mathfrak{p})$ we know that every element $a$ in $\mathfrak{B}$ can be written uniquely in the form $a=\sum_{Q} \lambda(Q) z_{Q}\left(z_{Q} \in \mathfrak{X}\right)$ (see Lemma 12 of [5]). Since $\mathfrak{B}=\mathfrak{B X}(\mathfrak{P}=\lambda(S(\mathfrak{p}))), \mathfrak{B Y}=\mathfrak{B Y}$ and it follows that $a \in \mathfrak{B} \mathfrak{Y}$ if and only if $z_{Q} \in \mathfrak{Y}$ for all $Q$. Our assertion is an immediate consequence of this fact.

Since $\overline{\mathfrak{X}}=\mathfrak{X} / \mathfrak{N}\left(\mathfrak{D}_{0}^{*}\right)$ is a finite-dimensional simple algebra, $\mathfrak{R}\left(\mathfrak{D}_{0}^{*}\right)=\cap \mathfrak{Y}$ where $\mathfrak{Y}$ runs over all maximal left ideals in $\mathfrak{X}$ containing $\mathfrak{R}\left(\mathfrak{D}_{0}^{*}\right)$. Therefore $\mathfrak{B N}\left(\mathfrak{D}_{0}^{*}\right)=\cap(\mathfrak{B Y})$ and so it would be sufficient to prove that

$$
\beta_{\nu}\left(\sum_{0 \leqq q \leqq h d} b^{q} z_{q}(y)\right) \in \mathfrak{B Y}[y]
$$

for every maximal left ideal $\mathfrak{Y}$ (in $\mathfrak{X}$ ) containing $\mathfrak{N}\left(\mathfrak{D}_{0}^{*}\right)$. Suppose then that the above statement is false for some $\mathfrak{Y}$. We shall often write $b(y)$ instead of $b$ to put in evidence the fact that, being an element of $A_{y}\left(\mathfrak{D}_{0}^{*}\right)$, it is a polynomial in $(y)$. Since $\mathfrak{V}$ is maximal it is clear that we can find $m_{i} \in C(1 \leqq i \leqq r)$ such that $\Gamma_{i}-m_{i} \in \mathfrak{V}(1 \leqq i \leqq r)$. Therefore

$$
\beta_{y}\left(\sum_{0 \leqq q \leqq h d} b^{q} z_{q}(y)\right) \equiv \sum_{1 \leqq j \leqq e} a_{j}(y) w_{j} \bmod \mathfrak{B Y}[y]
$$

where $w_{1}, \cdots, w_{e} \in \mathfrak{B X}^{\prime}$ and $a_{j}(y) \in C[y]$. (Here the notation is the same as in $\S 1$.) We may evidently assume that $w_{1}, \cdots, w_{e}$ are linearly independent $\bmod \mathfrak{B Y}$ and $a_{j}(y) \neq 0,1 \leqq j \leqq e$. Choose a linear function $\mu$ on $\mathfrak{c}$ such that $a_{1}(\mu) \neq 0$ and $\mu\left(\Gamma_{i}\right)+m_{i}, 1 \leqq i \leqq r$, are all integers. It is clear that this is possible. Then

$$
\beta_{\mu}\left(\sum_{0 \leqq q \leqq h d} b^{q}(\mu) z_{q}(\mu)-\sum_{1 \leqq j \leqq e} a_{j}(\mu) w_{j}\right) \in \mathfrak{B Y}
$$

since $w_{j} \in \mathfrak{P X} \mathfrak{X}^{\prime}$ and therefore $\beta_{\mu}\left(w_{j}\right)=w_{j}$. Here $b(\mu), z_{q}(\mu)$, and $a_{j}(\mu)$ are the values of $b(y), z_{q}(y)$, and $a_{j}(y)$ respectively under the substitution $y \rightarrow \mu$. Put $\mathfrak{V}_{\mu}=\beta_{-\mu}(\mathfrak{Y})$. Then since $\mathfrak{B V}=\mathfrak{B Y}$ it is clear that

$$
\sum_{0 \leqq q \leqq h d} b^{q}(\mu) z_{q}(\mu)-\sum_{1 \leqq j \leqq 。} a_{j}(\mu) w_{j} \in \mathfrak{B} \mathfrak{Y}_{\mu} .
$$

But $\sum_{1 \leqq j \leqq e} a_{j}(\mu) w_{j} \notin \mathfrak{B Y}$ because $a_{1}(\mu) \neq 0$. Hence

$$
\sum_{1 \leqq j \leqq 。} a_{j}(\mu) w_{j}=\beta_{-\mu}\left(\sum_{1 \leqq j \leqq} a_{j}(\mu) w_{j}\right) \notin \mathfrak{B} \mathfrak{Y}_{\mu}
$$

and so

$$
\sum_{0 \leqq q \leqq h d} b^{q}(\mu) z_{q}(\mu) \notin \mathfrak{B} \mathfrak{Y}_{\mu} .
$$

Let $\mathfrak{D}_{0}^{\prime *}$ be the equivalence class of the representation of $\mathfrak{t}^{\prime}$ defined by 
any representation of $\mathfrak{f}$ in $\mathfrak{D}_{0}^{*}$. It is clear that $\mathfrak{D}_{0}^{\prime *}$ is an irreducible class and since $\mathfrak{D}_{0}^{*} \in \Omega_{F}^{*}, \mathfrak{D}_{0}^{*} \in \Omega_{F}^{\prime}$. (See $\$ 1$ for notation.) Moreover $\Gamma_{i}-\left(\mu_{i}+m_{i}\right) \in \mathfrak{Y}_{\mu}$ $\left(\mu_{i}=\mu\left(\Gamma_{i}\right), 1 \leqq i \leqq r\right)$ and $\mu_{i}+m_{i}$ are all integers. Let $\mathfrak{D}_{0}$ be the class of the natural representation of $\mathfrak{t}$ on $\mathfrak{X} / \mathfrak{Y}_{\mu}$. In view of our choice of the base $\left(\Gamma_{1}, \cdots, \Gamma_{r}\right)$ it follows from the arguments of $\$ 1$ that $\mathfrak{D}_{0}$ occurs in some finite-dimensional representation of $\mathfrak{g}$. Therefore from Lemma 1 there exists a. left ideal $\mathfrak{M}$ in $\mathfrak{B}$ such that $\mathfrak{M} \supset \mathfrak{Y}_{\mu}$, $\operatorname{dim} \mathfrak{B} / \mathfrak{M}<\infty$, and $\sum_{q} b^{q}(\mu) z_{q}(\mu) \notin \mathfrak{M}$. Since $\mathfrak{g}$ is semisimple, we may assume that $\mathfrak{M}$ is a maximal left ideal in $\mathfrak{B}$. Let $a \rightarrow \bar{a}$ denote the natural mapping and $\nu$ the natural representation of $\mathfrak{B}$ on $\mathfrak{B} / \mathfrak{M}=\overline{\mathfrak{B}}$. Then $\nu\left(\sum_{q} b^{q}(\mu) z_{q}(\mu)\right) \overline{1} \neq 0$. Let $\psi \neq 0$ be an element in $\overline{\mathfrak{B}}$ belonging to the highest weight $\Lambda$ of $\nu$. We "extend" $\nu$ to a representation of the group $G$ on $\overline{\mathfrak{B}}$. Also we define a representation $\nu^{*}$ of $K^{*}$ by setting $\nu^{*}\left(v^{*}\right)$ $=e^{-(\mu \Gamma(v))} \nu(v)(v \in K)$. It is obvious that $\overline{1}$ transforms according to $\mathfrak{D}_{0}^{*}$ under $\nu^{*}$. Put

$$
E=d\left(\mathfrak{D}_{0}^{*}\right) \int_{\mathrm{K}^{*}} \chi \mathfrak{D}_{0}^{*}\left(v^{*-1}\right) \nu^{*}\left(v^{*}\right) d v^{*}
$$

where $\chi \mathfrak{D}_{0}^{*}$ is the character of $K^{*}$ corresponding to the class $\mathfrak{D}_{0}^{*}$. Then

$$
\nu(x) E \psi=d\left(\mathfrak{D}_{0}^{*}\right) \int_{K^{*}} \chi \mathfrak{D}_{0}^{*}\left(v^{*-1}\right) \nu(x) \nu^{*}\left(v^{*}\right) \psi d v^{*} .
$$

But $x v=v_{x} \exp H\left(x, v^{*}\right) \cdot n$ where $n \in N(x \in G, v \in K)$ (the notation is the same as in $[5, \S 12])$. Since $\psi$ belongs to the highest weight $\Lambda, \nu(n) \psi=\psi$ and therefore $\nu(x v) \psi=e^{\Delta\left(H\left(x, v^{*}\right)\right)} \nu\left(v_{x}\right) \psi$. So we get

$$
\nu(x) E \psi=d\left(\mathfrak{D}_{0}^{*}\right) \int_{K^{*}} \chi \mathfrak{D}_{0}^{*}\left(v^{*-1}\right) e^{\mu\left(\Gamma\left(x, v^{*}\right)\right)} e^{\Lambda\left(H\left(x, v^{*}\right)\right)} \nu^{*}\left(v_{x}^{*}\right) \psi d v^{*} .
$$

Now $E^{2}=E$ and $E \nu^{*}\left(v^{*}\right)=\nu^{*}\left(v^{*}\right) E$. Therefore

$$
E \nu(x) E \psi=d\left(\mathfrak{D}_{0}^{*}\right) \int_{K^{*}} \chi \mathfrak{D}_{0}^{*}\left(\left(v_{x-1}^{*}\right)^{-1}\right) e^{-\mu\left(\Gamma\left(x^{-1}, v^{*}\right)\right)} e^{-(\Lambda+2 \rho)\left(H\left(x^{-1}, v^{*}\right)\right)} \nu^{*}\left(v^{*}\right) \psi d v^{*}
$$

if we take into account the relation $d v_{x^{-1}}^{*}=e^{-2 \rho\left(\boldsymbol{H}\left(x^{-1}, v^{*}\right)\right)} d v^{*}$ (see $\left.[5, \S 11]\right)$. Now if we recall the definition of $\eta_{u, y}(a)=\eta_{u, y}^{\mathfrak{D}_{0}^{*}}(a)(a \in \mathfrak{B})$ it follows immediately that

$$
E \nu(a) E \psi=\gamma\left(\eta_{\Lambda_{\mathfrak{p}, \mu}}(a)\right) \quad(a \in \mathfrak{B}) .
$$

Here $\Lambda_{\mathfrak{p}}$ is the restriction of $\Lambda$ on $\mathfrak{h}_{\mathfrak{p}}$ and $\boldsymbol{\gamma}$ is the linear mapping of $\overline{\mathfrak{X}}$ into $E \overline{\mathfrak{B}}$ defined by $\gamma(\bar{z})=\nu^{*}(z) E \psi(z \in \mathfrak{X})$. (It is convenient to denote the corresponding representations of $K^{*}, \mathfrak{f}$, and $\mathfrak{X}$ by the same symbol $\nu^{*}$.)

Extend $\nu$ to a representation of $\mathfrak{B}[u, y]$ by setting $\nu\left(y_{i}\right)=\mu\left(\Gamma_{i}\right) \nu(1)$ $(1 \leqq i \leqq r)$ and $\nu\left(u_{i}\right)=\Lambda\left(H_{i}\right) \nu(1) \quad(1 \leqq i \leqq p)$. Now if $a(y) \in A_{y}\left(\mathfrak{D}_{0}^{*}\right)$, $\beta_{-\mu}\left(\mathfrak{N}\left(\mathfrak{D}_{0}^{*}\right)\right) a(\mu) \subset \mathfrak{B} \beta_{-\mu}\left(\mathfrak{N}\left(\mathfrak{D}_{0}^{*}\right)\right)$. But it is clear that $\beta_{-\mu}\left(\mathfrak{N}\left(\mathfrak{D}_{0}^{*}\right)\right)=\mathfrak{N}\left(\mathfrak{D}_{0}\right)$ and 
$E \overline{\mathfrak{B}}$ is exactly the set of those elements in $\overline{\mathfrak{B}}$ which transform under $\nu(\mathfrak{l})$ according to $\mathfrak{D}_{0}$. From this it follows that $E \nu(a(y)) E=\nu(a(y)) E$. Hence

$$
\begin{aligned}
\nu\left(b(y) \beta_{-y}\left(z_{i}\right)-\sum_{j=1}^{r} c_{i j}(u, y) \beta_{-y}\left(z_{j}\right)\right) & E \psi \\
& =E \nu\left(b(\mu) \beta_{-\mu}\left(z_{i}\right)-\sum_{j=1}^{r} c_{i j}\left(\Lambda_{\mathfrak{p}}, \mu\right) \beta_{-\mu}\left(z_{j}\right)\right) E \psi \\
& =\gamma\left(\eta_{\Lambda_{\mathfrak{p}, \mu}}\left(b(\mu) \beta_{-\mu}\left(z_{i}\right)-\sum_{j=1}^{r} c_{i j}\left(\Lambda_{\mathfrak{p}, \mu}\right) \beta_{-\mu}\left(z_{j}\right)\right) .\right.
\end{aligned}
$$

But we know that

$$
\eta_{u, y}\left(b(y) \beta_{-y}\left(z_{i}\right)-\sum_{j} c_{i j}(u, y) \beta_{-y}\left(z_{j}\right)\right)=0 .
$$

Hence

$$
\nu\left(b(y) \beta_{-y}\left(z_{i}\right)-\sum_{j} c_{i j}(u, y) \beta_{-y}\left(z_{j}\right)\right) E \psi=0
$$

or

$$
\nu(b(\mu)) \psi_{i}-\sum_{j} c_{i j}\left(\Lambda_{p, \mu}\right) \psi_{j}=0 \quad(1 \leqq i \leqq d)
$$

where $\psi_{i}=\nu\left(\beta_{-\mu}\left(z_{i}\right)\right) E \psi=\nu^{*}\left(z_{i}\right) E \psi$. Put $g(T)=\operatorname{det}\left(\delta_{i j} T-c_{i j}\left(\Lambda_{\mathfrak{p}, \mu}\right)\right)_{l \leqq i, j} \leqq_{d}$. Then $g(T)$ is a polynomial in $T$ with coefficients in $C$ and it is obvious that $\nu(g(b(\mu))) \psi_{i}=0,1 \leqq i \leqq d$. On the other hand $\mathfrak{g}=\mathfrak{l}+\mathfrak{h}_{\mathfrak{p}}+\mathfrak{n}\left(\mathfrak{n}=\sum_{\alpha \in P_{+}} C X_{\alpha}\right)$ and $\nu(\mathfrak{n}) \psi=\{0\}$ while $\nu\left(\mathfrak{h}_{\mathfrak{p}}\right) \psi \subset C \psi$. Moreover every element in $\mathfrak{B}$ can be written as a linear combination of elements of the form $z a n$ where $z \in \mathfrak{X}$ and $a$ and $n$ are products of elements in $\mathfrak{h}_{\mathfrak{p}}$ and $\mathfrak{n}$ respectively (see Lemma 12 of $[5])$. Hence

$$
E \overline{\mathfrak{B}}=E \nu(\mathfrak{B}) \psi=E \nu(\mathfrak{X}) \psi=\nu(\mathfrak{X}) E \psi=\nu^{*}(\mathfrak{X}) E \psi .
$$

Since $z_{1}, \cdots, z_{d}$ is a base for $\mathfrak{X} \bmod \mathfrak{R}\left(\mathfrak{D}_{0}^{*}\right)$ it follows that $E \overline{\mathfrak{B}}$ is spanned by $\psi_{i}=\nu^{*}\left(z_{i}\right) E \psi, 1 \leqq i \leqq d$. Moreover as $\overline{1}$ transforms under $\nu^{*}$ according to $\mathfrak{D}_{0}^{*}, \overline{1} \in E \bar{B}$ and so $\nu\left(g(b(\mu)) \overline{1}=0\right.$. But it is obvious that $g(T)=f\left(T, \Lambda_{\mathfrak{p}}, \mu\right)$ where $f\left(T, \Lambda_{\mathfrak{p}}, \mu\right)$ is the value of $f(T, u, y)$ under the substitution $u \rightarrow \Lambda_{\mathfrak{p}}$, $y \rightarrow \mu$. Let $F(T, \Lambda, \mu)$ denote the value of $F(T, x, y)$ under the substitution $x \rightarrow \Lambda$ (i.e. $x_{i} \rightarrow \Lambda\left(H_{i}\right), 1 \leqq i \leqq l$ ) and $y \rightarrow \mu$. Since $f^{(1)}(T, x, y)$ is one of the factors of $F(T, x, y), g(T)$ divides $F(T, \Lambda, \mu)$. Hence $\nu(F(b(\mu), \Lambda, \mu)) \overline{1}=0$. On the other hand

$$
F(T, \Lambda, \mu)=\sum_{0 \leqq q \leqq h d} T^{q} \chi_{\Delta}\left(z_{q}(\mu)\right)
$$

where $\chi_{\Lambda}\left(z_{q}(\mu)\right)$ is the value of $\chi_{x}\left(z_{q}(\mu)\right)$ under the substitution $x \rightarrow \Lambda$. Since $\nu$ 
is an irreducible representation with the highest weight $\Lambda$ we know that (see the proof of Lemma 36 of [3])

$$
\nu\left(z_{q}(\mu)\right)=\chi_{\Lambda}\left(z_{q}(\mu)\right) \nu(1) .
$$

Hence

$$
\nu(F(b(\mu), \Lambda, \mu))=\sum_{0 \leqq q \leqq h d} \nu\left(b^{q}(\mu)\right) \chi_{\Lambda}\left(z_{q}(\mu)\right)=\nu\left(\sum_{0 \leqq q \leqq h d} b^{q}(\mu) z_{q}(\mu)\right) .
$$

Therefore

$$
\nu\left(\sum_{0 \leqq q \leqq h d} b^{q}(\mu) z_{q}(\mu)\right) \overline{1}=0 .
$$

Since this contradicts our earlier result the lemma is proved.

In order to derive the consequences of the above lemma we need the following simple result.

Lemma 14. Let $\mathcal{A}$ be an associative algebra and $\sigma_{1}, \cdots, \sigma_{r}, \pi$ a finite set of finite-dimensional representations of $\mathcal{A}$. Let $\sigma$ be the direct sum of $\sigma_{i}, 1 \leqq i \leqq r$. Suppose $\pi$ is irreducible and $\pi(a)=0$ whenever $\sigma(a)=0(a \in \mathcal{A})$. Then there exists an index $i$ such that $\pi(a)=0$ whenever $\sigma_{i}(a)=0$.

Obviously $\sigma(\mathcal{A})$ is a finite-dimensional associative algebra. Hence $\sigma(\dot{\mathcal{A}})$ $=S_{0}+\mathcal{N}_{0}$ where $\mathcal{N}_{0}$ is the radical and $S_{0}$ a semisimple subalgebra of $\sigma(\mathcal{A})$. Let $S$ and $\mathcal{N}$ be the complete inverse images of $S_{0}$ and $\mathcal{N}_{0}$ respectively under $\sigma$. Since $\sigma(a) \rightarrow \pi(a)(a \in \mathcal{A})$ is an irreducible representation of $\sigma(\mathcal{A} \mathcal{A})$ it is obvious that $\pi(\mathcal{N})=\{0\}$. Now consider the representation $\phi: \sigma(s) \rightarrow \pi(s)$ $(s \in S)$ of $\sigma(\mathcal{S})$. Since $\sigma(\mathcal{S})$ is semisimple and $\pi$ is irreducible, $\phi$ must be equivalent to some irreducible component of the identical representation $\sigma(s) \rightarrow \sigma(s)$. Therefore since $\sigma$ is the direct sum of $\sigma_{i}, \phi$ is equivalent to an irreducible component of one of the representations $\sigma(s) \rightarrow \sigma_{i}(s)$. Hence we can choose an index $i$ such that $\sigma_{i}(s)=0$ implies $\pi(s)=0$. Now suppose $\sigma_{i}(a)=0(a \in \mathcal{C})$. Let $a=s+n(s \in \mathcal{S}, n \in \mathcal{N})$. Then $\sigma_{i}(s)=-\sigma_{i}(n) \in \sigma_{i}(\mathcal{N})$. However $\sigma_{i}(\mathcal{S})$ is a semisimple algebra and $\sigma_{i}(\mathcal{N})$ a nilpotent ideal in $\sigma_{i}(\mathcal{A})$. Therefore $\sigma_{i}(S) \cap \sigma_{i}(\mathcal{N})=\{0\}$. Hence $\sigma_{i}(s)=0$ and therefore $\pi(a)=\pi(s+n)$ $=\pi(s)=0$. Thus $\sigma_{i}(a)=0$ implies $\pi(a)=0$ and so our lemma is proved.

We shall now apply Lemma 13 to the theory of representations of $\mathfrak{B}$. Let $\pi$ be a representation of $\mathfrak{B}$ on a vector space $V$. For any $\mathfrak{D} \in \Omega$ let $V_{\mathfrak{D}}$ denote the subspace consisting of all those elements in $V$ which transform under $\pi(\mathfrak{l})$ according to $\mathfrak{D}$. Let $\mathfrak{R}(\mathfrak{D})$ denote the kernel (in $\mathfrak{X}$ ) of any representation of $\mathfrak{X}$ in $\mathfrak{D}$. As before, we shall say that $\pi$ is quasi-simple if $V$ $=\sum_{\mathbb{E} \in \Omega} V_{D}$ and there exists a homomorphism $\chi$ of $\mathcal{Z}$ into $C$ such that $\pi(z) \phi=\chi(z) \phi$ for all $z \in \mathbb{Z}$ and $\phi \in V . \chi$ is then called the infinitesimal character of $\pi$. Consider the mapping $z \rightarrow \chi_{x}(z)(z \in Z)$ of $Z$ into $C[x]$ which we have already discussed above. We have seen in [3, Part III] that there exists 
a linear function $\Lambda$ on $\mathfrak{h}$ such that $\chi(z)=\chi_{\Lambda}(z)(z \in Z)$ where $\chi_{\Lambda}(z)$ is the value of $\chi_{x}(z)$ under the substitution $x \rightarrow \Lambda$. As usual we say that $\pi$ is irreducible if $\pi(\mathfrak{B}) \psi=V$ for every nonzero $\psi$ in $V$.

Let $\pi$ be a quasi-simple irreducible representation of $\mathfrak{B}$ on a vector space $V$ such that $V_{\mathfrak{D}_{0}} \neq\{0\}$ for some $\mathfrak{D}_{0} \in \Omega_{F}$. Choose a linear function $\mu$ on $\mathfrak{c}$ such that $\Gamma-\mu(\Gamma) \in \mathfrak{R}\left(\mathfrak{D}_{0}\right)$ for all $\Gamma \in \mathfrak{c}$. It is clear that there exists a class $\mathfrak{D}_{0}^{*} \in \Omega^{*}$ such that $\mathfrak{R}\left(\mathfrak{D}_{0}^{*}\right)=\beta_{\mu}\left(\mathfrak{R}\left(\mathfrak{D}_{0}\right)\right)$. Let $A\left(\mathfrak{D}_{0}\right)$ be the set of all $a \in \mathfrak{B}$ for which $\mathfrak{N}\left(\mathfrak{D}_{0}\right) a \subset \mathfrak{B} \mathfrak{N}\left(\mathfrak{D}_{0}\right)$.

Lemma 15. There exists a linear function $\Lambda$ on $\mathfrak{h}$ with the following two properties:

(1) $\chi_{\Lambda}$ is the infinitesimal character of $\pi$.

(2) Let $\Lambda_{\mathfrak{p}}$ denote the restriction of $\Lambda$ on $\mathfrak{h}_{\mathfrak{p}}$. Suppose $a$ is an element in $A\left(\mathfrak{D}_{0}\right)$ such that $\eta_{\Lambda_{\mathfrak{p}, \mu}}^{\mathfrak{D}^{*}}(a z)=0$ for all $z \in \mathfrak{X}$. Then $\pi(a) \psi=0$ for every $\psi \in V_{D_{0}}$.

Let $A_{1}$ be the set of all $a \in \mathfrak{B}_{1}=\mathfrak{B}[y]$ such that $\beta_{-y}\left(\mathfrak{R}\left(\mathfrak{D}_{0}^{*}\right)\right) a$ $\subset \mathfrak{B}_{1} \beta_{-y}\left(\mathfrak{N}\left(\mathfrak{D}_{0}^{*}\right)\right)\left(A_{1}=A_{y}\left(\mathfrak{D}_{0}^{*}\right)\right.$ in the notation of Lemma 12$)$. Let $b$ be any element in $A_{1}$. Since $\mathfrak{D}_{0} \in \Omega_{F}$ and since $\mathfrak{c} \subset \mathfrak{R}\left(\mathfrak{D}_{0}^{*}\right)$ it follows from the argument given in $\S 1$ that $\mathfrak{D}_{0}^{*} \in \Omega_{F}^{*}$ and therefore

$$
\beta_{y}\left(\sum_{0 \leqq q \leqq h d} b^{q_{z_{q}}}(y)\right) \in \mathfrak{B N}\left(\mathfrak{D}_{0}^{*}\right)[y]
$$

in the notation of Lemma 13. Hence

$$
\sum_{0 \leqq q \leqq h d} b^{q} z_{q}(y) \in \mathfrak{B}_{1} \beta_{-y}\left(\mathfrak{N}\left(\mathfrak{D}_{0}^{*}\right)\right) .
$$

Extend $\pi$ to a representation of $\mathfrak{B}_{1}$ by putting $\pi\left(y_{i}\right)=\mu\left(\Gamma_{i}\right) \pi(1)(1 \leqq i \leqq r)$. Then if $\psi \in V_{\mathfrak{D}_{0}}$

$$
\pi\left(\beta_{-y}\left(\mathfrak{N}\left(\mathfrak{D}_{0}^{*}\right)\right)\right) \psi=\pi\left(\beta_{-\mu}\left(\mathfrak{N}\left(\mathfrak{D}_{0}^{*}\right)\right)\right) \psi=\pi\left(\mathfrak{N}\left(\mathfrak{D}_{0}\right)\right) \psi=\{0\} .
$$

Hence

$$
\pi\left(\sum_{0 \leqq q \leqq h d} b^{q} z_{q}(y)\right) \psi=0 .
$$

It is obvious that $V_{D_{0}}$ is invariant under $\pi\left(A_{1}\right)$. Hence we get a representation $\nu$ of $A_{1}$ on $V_{\mathfrak{D}_{0}}$. Now $A_{1} \supset \mathfrak{X}$ and we know from Corollary 2 to Theorem 2 that $V_{D_{0}}$ is irreducible under $\pi(\mathfrak{X})$. Therefore $\nu$ is an irreducible representation of $A_{1}$ on $V_{D_{0}}$.

Let $\Lambda$ be a linear function on $\mathfrak{h}$ such that $\chi_{\Lambda}$ is the infinitesimal character of $\pi$. We use the notation of Lemma 13 and consider the polynomials $f(T, u, y), f^{(\sigma)}(T, x, y) \quad(\sigma \in W)$ corresponding to the element $b \in A_{1}$. Let $f^{(\sigma)}(T, \Lambda, \mu)$ denote the value of $f^{(\sigma)}(T, x, y)$ under the substitution $x \rightarrow \Lambda$, $y \rightarrow \mu$. Put $\Lambda^{\sigma}(H)=\Lambda\left(\sigma^{-1} H\right)+\rho\left(\sigma^{-1} H\right)-\rho(H)(H \in \mathfrak{h})$ and let $\Lambda_{\mathfrak{p}}^{\sigma}$ denote the 
restriction of $\Lambda^{\sigma}$ on $\mathfrak{h}_{\mathfrak{p}}$. Then it follows from the definition of $f^{(\sigma)}(T, x, y)$ that $f^{(\sigma)}(T, \Lambda, \mu)=f\left(T, \Lambda_{\mathfrak{p}}^{\sigma}, \mu\right)$ where $f\left(T, \Lambda_{\mathfrak{p}}^{\sigma}, \mu\right)$ is the value of $f(T, u, y)$ under the substitution $u \rightarrow \Lambda_{\mathfrak{p}}^{\sigma}, y \rightarrow \mu$. Therefore

$$
\begin{aligned}
0=\pi\left(\sum_{q} b^{q} z_{q}(y)\right) \psi & =\sum_{q} \chi_{\Lambda}\left(z_{q}(\mu)\right) \pi\left(b^{q}\right) \psi \\
& =\nu\left(\prod_{\sigma \in W} f^{(\sigma)}(b, \Lambda, \mu)\right) \psi \quad\left(\psi \in V_{\mathfrak{D}_{0}}\right) .
\end{aligned}
$$

Hence

$$
\nu\left(\prod_{\sigma \in W} f^{(\sigma)}(b, \Lambda, \mu)\right)=\nu\left(\prod_{\sigma \in W} f\left(b, \Lambda_{p}^{\sigma}, \mu\right)\right)=0 .
$$

We have the base $z_{1}, \cdots, z_{d}$ for $\mathfrak{X} \bmod \mathfrak{N}\left(\mathfrak{D}_{0}^{*}\right)$. Put $z_{i}^{\prime}=\beta_{-y}\left(z_{i}\right), 1 \leqq i \leqq d$. Let $\Re_{2}$ denote the set of all elements $a \in A_{1}[u]=A_{2}$ such that $\eta_{u, y}(a)=0\left(\eta_{u, y}\right.$ $\left.=\eta_{u, y}^{\mathfrak{D}_{0}^{*}}\right)$. We know from Lemma 5 that $\Re_{2}$ is a left ideal in $A_{2}$. Put

$$
\Re_{\Lambda_{\mathfrak{p}, \mu}^{\sigma}}^{\prime \sigma}=\Re_{2}+\sum_{1 \leqq i \leqq p} A_{2}\left(u_{i}-\Lambda^{\sigma}\left(H_{i}\right)\right)+\sum_{1 \leqq i \leqq r} A_{2}\left(y_{i}-\mu\left(\Gamma_{i}\right)\right) \quad(\sigma \in W) .
$$

For any $a \in A_{2}$ let $\eta_{\Lambda_{\mathfrak{p}, u}^{\sigma}}^{\prime}(a)$ denote the value of $\eta_{u, y}(a)$ under the substitution $u \rightarrow \Lambda_{\mathfrak{p}}^{\sigma}, y \rightarrow \mu$. It is clear that $\eta_{\Lambda_{\mathfrak{p}, \mu}^{\sigma}}^{\prime \sigma}(a)=0$ for $a \in \Re_{\Lambda_{\mathfrak{p}, \mu}^{\sigma}}^{\prime \sigma}$. Moreover we have seen (see p. 52) that $A_{2}=\Re_{2}+\sum_{i=1}^{a} C[u, y] z_{i}^{\prime}$. Hence

$$
A_{2}=\Re_{\Lambda_{\mathfrak{p}, \mu}^{\sigma}}^{\prime}+\sum_{1 \leqq i \leqq d} C z_{i}^{\prime}
$$

Therefore for any $a \in A_{2}$ we can choose $c_{i} \in C$ such that

$$
a \equiv \sum_{1 \leqq i \leqq d} c_{i} z_{i}^{\prime} \bmod \Re_{\Lambda_{\mathfrak{p}, \mu}^{\prime}}^{\prime}
$$

and so

$$
\eta_{\Lambda_{\mathfrak{p}, \mu}^{\sigma}}^{\prime}(a)=\sum_{1 \leqq i \leqq d} c_{i} \eta_{\Lambda_{\mathfrak{p}, \mu}^{\sigma}}^{\prime}\left(z_{i}^{\prime}\right)
$$

But, from Lemma $6, \eta_{u, y}\left(z_{i}^{\prime}\right)=\bar{z}_{i}$ where $z \rightarrow \bar{z}$ is the natural mapping of $\mathfrak{X}$ on $\overline{\mathfrak{X}}=\mathfrak{X} / \mathfrak{N}\left(\mathfrak{D}_{0}^{*}\right)$. Hence $\eta_{\Lambda_{\mathfrak{p}, \mu}^{\prime}}^{\prime}\left(z_{i}^{\prime}\right)=\bar{z}_{i}$ and therefore

$$
\eta_{\Lambda_{\mathfrak{p}, \mu}^{\sigma}}^{\prime}(a)=\sum_{1 \leqq i \leqq d} c_{i} \bar{z}_{i}
$$

So in particular if $\eta_{\Lambda_{\mathfrak{p}, \mu}^{\sigma}}^{\prime}(a)=0, c_{i}=0,1 \leqq i \leqq d$, and therefore $a \in \Re_{\Lambda_{\mathfrak{p}, u}^{\sigma}}^{\prime}$. This proves that an element $a \in A_{2}$ belongs to $\Re_{\Lambda_{\mathfrak{p}, \mu}}^{\prime}$ if and only if $\eta_{\Lambda_{\mathfrak{p}, \mu}^{\sigma}}^{\prime}(a)=0$. Since $\eta_{\Lambda_{\mathrm{p}, \mu}^{\sigma}}^{\sigma}\left(\sum_{i} c_{i} z_{i}\right)=\sum_{i} c_{i} \bar{z}_{i}^{\prime}\left(c_{i} \in C\right)$ we conclude that $z_{1}^{\prime}, \cdots, z_{r}^{\prime}$ are linearly independent over $C$ modulo $\Re_{\Lambda_{\mathfrak{p}, \mu}^{\sigma}}^{\prime}$. 
Put $\Re^{\prime}=\bigcap_{\sigma \in W} \Re_{\Lambda_{\mathfrak{p}, \mu}^{\sigma}}^{\prime}$. We have seen above that $A_{2}=\Re_{\Lambda_{\mathfrak{p}, \mu}^{\sigma}}^{\prime}+\sum_{i=1}^{d} C_{i} z_{i}^{\prime}$. Hence $\operatorname{dim} A_{2} / \mathfrak{R}_{\Lambda_{\mathfrak{p}, \mu}^{\prime}}^{\prime} \leqq d$ and therefore $\operatorname{dim} A_{2} / \Re^{\prime} \leqq h d$ where $h$ is the order of the Weyl group $W$. Let $\tau$ be the natural representation of $A_{2}$ on $A_{2} / \Re^{\prime}$. Suppose $\tau(b)=0$ for some $b \in A_{1}$. Then $b z_{i}^{\prime} \in \Re^{\prime} \subset \Re_{\Lambda_{\mathfrak{p}, \mu}^{\sigma}}^{\prime}(1 \leqq i \leqq d)$. But in the notation of Lemma 13

$$
b z_{i}^{\prime}-\sum_{j} c_{i j}(u, y) z_{j}^{\prime} \in \Re_{2} \subset \Re_{\Lambda_{\mathfrak{p}, \mu}^{\sigma}}^{\prime}
$$

Since $b z_{i}^{\prime} \in \Re$ and $c_{i j}(u, y) z_{j}^{\prime}-c_{i j}\left(\Lambda_{\mathfrak{p}, \mu}^{\sigma}\right) z_{j}^{\prime} \in \Re_{\Lambda_{\mathfrak{p}, \mu}^{\sigma}}^{\prime}$ it follows that

$$
\sum_{j=1}^{d} c_{i j}\left(\Lambda_{\mathfrak{p}}^{\sigma}, \mu\right) z_{j}^{\prime} \in \Re_{\Lambda_{\mathfrak{p}, \mu}^{\mathfrak{g}} \text {. }}^{\prime}
$$

Therefore in view of the linear independence of $z_{j}^{\prime}$ over $C \bmod \Re_{\Lambda_{\mathfrak{p}, \mu}^{\sigma}}^{\prime \sigma}$ we conclude that $c_{i j}\left(\Lambda_{\mathfrak{p}, \mu}^{\sigma}\right)=0,1 \leqq i, j \leqq d$. But on the other hand

$$
f(T, u, y)=\operatorname{det}\left(T \delta_{i j}-c_{i j}(u, y)\right), \quad 1 \leqq i, j \leqq d .
$$

Therefore $f\left(T, \Lambda_{\mathfrak{p}}^{\sigma}, \mu\right)=T^{d}$ and

$$
0=\nu\left(\prod_{\sigma \in W} f\left(b, \Lambda_{p}^{\sigma}, \mu\right)\right)=\nu\left(b^{d h}\right)=0 .
$$

Hence $\tau(b)=0\left(b \in A_{1}\right)$ implies that $\nu(b)$ is nilpotent. Let $B_{1}$ be the set of all elements $a \in A_{1}$ such that $\tau(a)=0$. Then it follows that $\nu\left(B_{1}\right)$ is a two-sided ideal in $\nu\left(A_{1}\right)$ all of whose elements are nilpotent. But since $\nu$ is irreducible the algebra $\nu\left(A_{1}\right)$ is simple. Hence $\nu\left(B_{1}\right)=\{0\}$. This shows that $\tau(a)=0\left(a \in A_{1}\right)$ implies $\nu(a)=0$.

Let $\tau^{(\sigma)}$ denote the natural representation of $A_{1}$ on $A_{1} / \Re_{\Lambda_{\mathfrak{p}, \mu}}^{\prime \prime} \cong A_{2} / \Re_{\Lambda_{\mathfrak{p}, \mu}^{\sigma}}^{\prime \sigma}$ where $\Re_{\Lambda_{\mathfrak{p}, \mu}}^{\prime \sigma}=A_{1} \cap \Re_{\Lambda_{\mathfrak{p}, \mu}}^{\prime \sigma}$. Let $\tau_{0}$ be the direct sum of $\tau^{(\sigma)}(\sigma \in W)$. It is clear that $\tau_{0}(a) \stackrel{=}{=}\left(a \in A_{1}\right)$ if and only if $\tau(a)=0$. Therefore it is possible (from Lemma 14) to choose a $\sigma \in W$ such that $\tau^{(\sigma)}(a)=0\left(a \in A_{1}\right)$ implies $\nu(a)=0$. Since $\chi_{\Lambda}=\chi_{\Lambda^{\sigma}}$ (see $[3$, Theorem 5$]$ ) we may assume without loss of generality that $\sigma=1$.

For any $a \in A_{1}$ let $\phi(a)$ denote the value of $a$ under the substitution $y \rightarrow \mu$. We have seen in Lemma 12 that $\phi$ is a homomorphism of $A_{1}$ onto $A\left(\mathfrak{D}_{0}\right)$. Let $\Re_{\Lambda \mathfrak{p}, \mu}$ be the set of all $b \in A\left(\mathfrak{D}_{0}\right)$ such that $\eta_{\Lambda \mathrm{p}, \mu}(b)=0$. Let $\sigma$ be the natural representation of $A\left(\mathfrak{D}_{0}\right)$ on $A\left(\mathfrak{D}_{0}\right) / \Re_{\Lambda p, \mu}$. Suppose $\sigma(a)=0\left(a \in A\left(\mathfrak{D}_{0}\right)\right)$. Then $a \beta_{-\mu}\left(z_{i}\right) \in \Re_{\Lambda p, \mu}, 1 \leqq i \leqq d$. Choose $b \in A_{1}$ such that $a=\phi(b)$. Then $\phi\left(b z_{i}^{\prime}\right)$ $=a \beta_{-\mu}\left(z_{i}\right)$ and therefore $\eta_{\Lambda p, \mu}\left(\phi\left(b z_{i}^{\prime}\right)\right)=0,1 \leqq i \leqq d$. As we have seen above this implies that $b z_{i}^{\prime} \in \Re_{\Lambda \mathfrak{p}, \mu}^{\prime}, 1 \leqq i \leqq d$. Since $A_{2}=\sum_{i=1}^{d} C z_{i}^{\prime}+\Re_{\Lambda \mathfrak{p}, \mu}^{\prime}$ it follows that $b A_{2} \subset \Re_{\Lambda p, \mu}^{\prime}$ and so $\tau^{(1)}(b)=0$ where $\tau^{(1)}$ is the natural representation of $A_{1}$ on $A_{1} / \Re_{\Lambda \mathfrak{p}, \mu}^{\prime \prime}$. This in its turn implies that $\nu(b)=0$. But $\nu(b)=\nu(\phi(b))=\nu(a)$. Hence this proves $\nu(a)=0$ whenever $\sigma(a)=0$. Since it is obvious that $A\left(\mathfrak{D}_{0}\right)$ $=\Re_{\Lambda p, \mu}+\mathfrak{X}$ the lemma follows. 
Now consider the representation $\pi_{\Delta p, \mu}$ of $G$ on $L_{2}\left(K^{*}\right)$ defined by

$$
\pi_{\Lambda_{p}, \mu}(x) f\left(v^{*}\right)=e^{-\mu\left(\Gamma\left(x^{-1}, v^{*}\right)\right)} e^{-\left(\Lambda_{p}+2 \rho\right)\left(H\left(x^{-1}, v^{*}\right)\right)} f\left(v_{x^{-1}}^{*}\right) \quad\left(x \in G, v^{*} \in K^{*}\right) .
$$

Put $f_{0}\left(v^{*}\right)=d\left(\mathfrak{D}_{0}^{*}\right) \chi D_{0}{ }^{*}\left(v^{*-1}\right)$ where $\chi D_{0}^{*}$ is the character of the class $\mathfrak{D}_{0}^{*}$. For any $\mathfrak{D} \in \Omega$ let $\mathfrak{S}_{\mathfrak{D}}$ denote the set of those elements in $\mathfrak{S}=L_{2}\left(K^{*}\right)$ which transform under $\pi_{\Lambda \mathrm{p}, \mu}(K)$ according to $\mathfrak{D}$. Then we know that every element in $\sum_{\mathfrak{D} \in \Omega} \mathfrak{S}_{\mathfrak{D}}$ is well-behaved (see Lemma 34 of [5]). Let $b \rightarrow \pi_{\Lambda \mathfrak{p}, \mu}(b)(b \in \mathfrak{B})$ denote the corresponding representation of $\mathfrak{B}$ defined on $\sum_{\mathfrak{D} \in \Omega} \mathfrak{F}_{\mathfrak{D}}$ (see Lemma 9 of [5]). It is obvious that $f_{0} \in \mathfrak{S}_{\mathfrak{D}_{0}}$ and $\pi_{\Lambda p, \mu}(\mathfrak{X}) f_{0}=\mathfrak{S}_{\mathfrak{D}_{0}}$. Let $\tau$ be the left regular representation of $K^{*}$ on $\mathfrak{S}$. Put $\overline{\mathfrak{X}}=\mathfrak{X} / \mathfrak{N}\left(\mathfrak{D}_{0}^{*}\right)$ and let $\bar{z} \rightarrow \bar{\tau}(\bar{z})(\bar{z} \in \overline{\mathfrak{X}})$ denote the corresponding representation of $\overline{\mathfrak{X}}$ on $\mathfrak{S E D}_{0}$. Then we have seen in the proof of Lemma 5 that

$$
\pi_{\Lambda_{\mathfrak{p}, \mu}}(a) f_{0}=\bar{\tau}\left(\eta_{\Lambda_{\mathfrak{p}}, \mu}(a)\right) f_{0} \quad\left(a \in A\left(\mathfrak{D}_{0}\right)\right) .
$$

Moreover $\bar{\tau}(\bar{z}) f_{0} \neq 0(\bar{z} \in \overline{\mathfrak{X}})$ unless $\bar{z}=0$. Hence $\pi_{\Lambda \mathfrak{p}, \mu}(a) f_{0}=0$ if and only if $\eta_{\Lambda \mathrm{p}, \mu}(a)=0 \quad\left(a \in A\left(\mathfrak{D}_{0}\right)\right)$. Thus the natural representation of $A\left(\mathfrak{D}_{0}\right)$ on $A\left(\mathfrak{D}_{0}\right) / \Re_{\Lambda \mathfrak{p}, \mu}$ is equivalent to its representation on $\mathfrak{S}_{\mathfrak{D}_{0}}$ induced under $\pi_{\Lambda \mathfrak{p}, \mu}$.

Let $M^{*}$ be the analytic subgroup of $K^{*}$ corresponding to the subalgebra $\mathfrak{m}_{0}=\mathfrak{f}_{0} \cap\left(\mathfrak{h}_{\mathfrak{t}}+\sum_{\alpha \in P_{-}}\left(C X_{\alpha}+C X_{-\alpha}\right)\right)$. We know that $M^{*}$ is compact (see $[5, \S 12])$. Let $\tau^{\prime}$ be the right regular representation of $K^{*}$ on $\mathfrak{S}$ so that $\tau^{\prime}\left(u^{*}\right) f\left(v^{*}\right)=f\left(v^{*} u^{*}\right)\left(u^{*}, v^{*} \in K^{*} ; f \in \mathfrak{S}\right)$. Let $\mathfrak{D}_{0}^{\prime *}$ be the class dual to $\mathfrak{D}_{0}^{*}$. It is obvious that every element in $\mathfrak{S}_{\mathfrak{D}_{0}}$ transforms under $\tau\left(K^{*}\right)$ according to $\mathfrak{D}_{0}^{*}$ and therefore under $\tau^{\prime}\left(K^{*}\right)$ according to $\mathfrak{D}_{0}^{\prime *}$. Let $\omega^{*}$ be the set of all equivalence classes of finite-dimensional simple representations of $M^{*}$. Denote by $\delta_{1}^{\prime *}, \ldots, \delta_{s}^{\prime *}$ all the distinct elements in $\omega^{*}$ which occur in the reduction of $\mathfrak{D}_{0}^{* \prime}$ with respect to $M^{*}$. Let $\xi_{j}^{\prime}$ be the character of $M^{*}$ corresponding to $\delta_{j}^{\prime *}$. Put

$$
E_{j}^{\prime}=d_{j} \int_{M^{*}} \xi_{j}^{\prime}\left(m^{*-1}\right) \tau^{\prime}\left(m^{*}\right) d m^{*}
$$

where $d_{j}$ is the degree of any representation in $\delta_{j}^{\prime *}$ and the Haar measure $d m^{*}$ on $M^{*}$ is so normalised that $\int_{M^{*}} d m^{*}=1$. It follows from the theory of compact groups that $E_{i}^{\prime} E_{j}^{\prime}=\delta_{i j} E_{j}^{\prime}(1 \leqq i, j \leqq s)$ and $f=E_{1}^{\prime} f+\cdots+E_{s}^{\prime} f$ $\left(f \in \mathfrak{S}_{\mathcal{D}_{0}}\right)$. We know moreover (see Lemma 37 of [5]) that $\tau^{\prime}\left(m^{*}\right)$ commutes with $\pi_{\Lambda \mathfrak{p}, \mu}(x)\left(m^{*} \in M^{*}, x \in G\right)$. Therefore the subspaces $\mathfrak{S}_{j}=E_{j}^{\prime} \mathfrak{S}, 1 \leqq j \leqq s$, are closed invariant subspaces of $\mathfrak{S}$ and $\mathfrak{S E D}_{0} \cap \mathfrak{S}_{j}=E_{j}^{\prime} \mathfrak{S E D}_{\mathfrak{D}_{0}}, \mathfrak{S E D}_{0}$ $=\sum_{j=1}^{s} E_{j}^{\prime} \mathfrak{S}_{\mathfrak{D}_{0}}$. Let $\sigma_{j}$ denote the representation of $A\left(\mathfrak{D}_{0}\right)$ induced on $E_{j}^{\prime} \mathfrak{S E D}_{\mathcal{D}_{0}}$ under $\pi_{\Lambda \mathfrak{p}, \mu}$. From Lemma 14 we conclude that there exists an index $j$ (say $j=1$ ) such that $\left(^{3}\right) \nu(a)=0$ whenever $\sigma_{1}(a)=0\left(a \in A\left(\mathfrak{D}_{0}\right)\right)$. We now drop the index 1 and write $E^{\prime}, \delta^{\prime *}$, and $\sigma$ instead of $E_{1}^{\prime}, \delta_{1}^{\prime *}$, and $\sigma_{1}$ respec-

(3) We recall that $\nu$ is the representation of $A\left(\mathfrak{D}_{0}\right)$ on $V_{\mathfrak{D}_{0}}$. 
tively. Let $\xi_{i j}, 1 \leqq i, j \leqq d$, be the matrix elements of an irreducible unitary

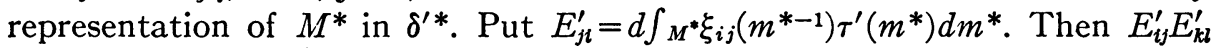
$=E_{i l}^{\prime} \delta_{j k}$ and $E^{\prime}=\sum_{i=1}^{d} E_{i \mathfrak{t}}^{\prime}$. Put $\mathfrak{S}_{i}=E_{i l}^{\prime} \mathfrak{S}_{\mathfrak{D}_{0}}=E_{i i}^{\prime} E^{\prime} \mathfrak{S}_{\mathfrak{D}_{0}}$. Then again since $E_{\imath \imath}^{\prime}$ commutes with $\pi_{\Lambda \mathrm{p}, \mu}(x)(x \in G), \mathfrak{S}_{i}$ is stable under $\sigma\left(A\left(\mathfrak{D}_{0}\right)\right)$. Let $\sigma_{i}$ denote the representation of $A\left(\mathfrak{D}_{0}\right)$ induced on $\mathfrak{S}_{i}$. We conclude once more that there exists an index $i$ (say $i=1$ ) such that $\nu(a)=0$ whenever $\sigma_{1}(a)=0$ $\left(a \in A\left(\mathfrak{D}_{0}\right)\right)$. Since $\nu$ is irreducible we can find two subspaces $U_{1}, U_{2}$ of $\mathfrak{S}_{1}$ $\left(U_{1} \supset U_{2}\right)$ which are both stable under $\pi_{\Lambda \mathfrak{p}, \mu}\left(A\left(\mathfrak{D}_{0}\right)\right)$ and such that the representation of $A\left(\mathfrak{D}_{0}\right)$ induced on $U_{1} / U_{2}$ is equivalent to $\nu$. Choose an element $f_{00}$ in $U_{1}$ which is not in $U_{2}$. Since $f_{00} \in E_{11}^{\prime} \mathfrak{S}_{\mathfrak{D}_{0}}$ the space spanned by $\tau^{\prime}\left(m^{*}\right) f_{00}$ $\left(m^{*} \in M^{*}\right)$ is irreducible and transforms according to $\delta^{*}$ under $\tau^{\prime}\left(M^{*}\right)$. Moreover there exists an element $\psi_{00} \in V_{\mathfrak{D}_{0}}\left(\psi_{00} \neq 0\right)$ with the property that $\nu(a) \psi_{00}$ $=0\left(a \in A\left(\mathfrak{D}_{0}\right)\right)$ if and only if $\pi_{\Lambda p, \mu}(a) f_{00} \in U_{2}$. Let $B$ be the set of those $a$ $\in A\left(\mathfrak{D}_{0}\right)$ for which $\nu(a) \psi_{00}=0$. Then $B$ is a maximal left ideal in $A\left(\mathfrak{D}_{0}\right)$. We shall now prove that $\pi_{\Lambda p, \mu}(\mathfrak{B} B) f_{00} \neq \pi_{\Lambda \mathrm{p}, \mu}(\mathfrak{B}) f_{00}$. For otherwise $f_{00}$ $=\sum_{i=1}^{t} \pi_{\Lambda \mathrm{p}, \mu}\left(a_{i} b_{i}\right) f_{00}$ where $a_{i} \in \mathfrak{B}, b_{i} \in B$. Consider the natural mapping $b \rightarrow b^{*}$ and the natural representation $\pi^{*}$ of $\mathfrak{B}$ on $\mathfrak{B}^{*}=\mathfrak{B} / \mathfrak{B N}\left(\mathfrak{D}_{0}\right)$. We know (see Theorem 1 of [5]) that $\mathfrak{B}^{*}=\sum \mathfrak{D} \in \Omega_{\mathfrak{D}} \mathfrak{B}_{\mathfrak{D}}^{*}$ where $\mathfrak{B}_{\mathfrak{D}}^{*}$ is the set of those elements in $\mathfrak{B}^{*}$ which transform according to $\mathfrak{D}$ under $\pi^{*}(\mathfrak{f})$. Pick a set of classes $\mathfrak{D}_{1}, \cdots, \mathfrak{D}_{N} \in \Omega\left(\mathfrak{D}_{j} \neq \mathfrak{D}_{0}, 1 \leqq j \leqq N\right)$ such that $a_{i}{ }^{*}, 1 \leqq i \leqq t$, are all contained in $\mathfrak{B}_{\mathfrak{D}_{0}}^{*}+\mathfrak{B}_{\mathfrak{D}_{1}}^{*}+\cdots+\mathfrak{B}_{\mathfrak{D}_{N}}^{*}$. From Lemma 4 we can choose $\epsilon \in \mathfrak{X}$ such that $\pi^{*}(e) b^{*}=b^{*}$ if $b^{*} \in \mathfrak{B}_{\mathfrak{D}_{0}}^{*}$ and $\pi^{*}(e) b^{*}=0$ if $b^{*} \in \mathfrak{B}_{\mathfrak{D}_{j}}^{*}, 1 \leqq j \leqq N$. From this it follows immediately that $f_{00}=\pi_{\Lambda \mathrm{p}, \mu}(e) f_{00}=\sum_{i=1}^{t} \pi_{\Lambda \mathrm{p}, \mu}\left(e a_{i} b_{i}\right) f_{00}$. Moreover $\left(e a_{i}\right)^{*} \in \mathfrak{B}^{*} \mathfrak{D}_{0}$ and therefore $e a_{i} \in A\left(\mathfrak{D}_{0}\right)$. Since $B$ is a left ideal in $A\left(\mathfrak{D}_{0}\right)$, $e a_{i} b_{i} \in B$. Hence $f_{00} \in \pi_{\Lambda \mathrm{p}, \mu}(B) f_{00} \subset U_{2}$. Since this contradicts our choice of $f_{00}$ we conclude that $\pi_{\Lambda \mathfrak{p}, \mu}(\mathfrak{B} B) f_{00} \neq \pi_{\Lambda \mathfrak{p}, \mu}(\mathfrak{B}) f_{00}$.

Let $\mathscr{X}_{1}$ be the left ideal consisting of all $b \in \mathfrak{B}$ for which $\pi_{\Lambda p, \mu}(b) f_{00}=0$. Then the above result shows that $\mathfrak{B B}+\mathcal{X}_{1} \neq \mathfrak{B}$. By Zorn's lemma we can find a maximal left ideal $\mathscr{X}$ in $\mathscr{B}$ containing $\mathfrak{B} B+\mathscr{X}_{1}$. Then $\pi_{\Lambda \mathrm{p}, \mu}(\mathscr{X}) f_{00}$ $\neq \pi_{\Delta p, \mu}(\mathfrak{B}) f_{00}$. Let $\mathfrak{S}^{\prime}$ and $\mathfrak{S}^{\prime \prime}$ respectively be the closures in $\mathfrak{S}$ of the linear spaces $\pi_{\Lambda \mathrm{p}, \mu}(\mathfrak{B}) f_{00}$ and $\pi_{\Lambda \mathrm{p}, \mu}(\mathscr{H} \mathcal{C}) f_{00}$. It follows from Theorem 5 of [5] that $\mathfrak{S}^{\prime}, \mathfrak{S}^{\prime \prime}$ are both invariant under $\pi_{\Lambda p, \mu}(G)$ and $\mathfrak{S}^{\prime \prime}$ is maximal in $\mathfrak{S}^{\prime}$. We regard $U=\mathfrak{S}^{\prime} / \mathfrak{S}^{\prime \prime}$ as a Hilbert space in the usual way and consider the representation $\gamma$ of $G$ induced on $U$ under $\pi_{\Lambda p, \mu}$. Then $\gamma$ is irreducible. Let $f_{00}^{*}$ denote the image of $f_{00}$ in $U$ under the natural mapping of $\mathfrak{S}^{\prime}$ on $U$. Then $f_{00}^{*}$ transforms under $\gamma(K)$ according to $\mathfrak{D}_{0}$ and therefore it is well-behaved under $\gamma$ (see Lemma 34 of [5]). Moreover $\gamma(\mathscr{P C}) f_{00}^{*}=\{0\}$. Let $Z_{x}$ be the set of all elements of the form $z-\chi(z)(z \in \mathbb{Z})$. Then $B \supset \mathfrak{B}_{x}$ and therefore $\mathfrak{H} \supset \mathfrak{B N}\left(\mathfrak{D}_{0}\right)$ $+\mathscr{B} \mathfrak{B}_{x}$. Moreover since $B$ is a maximal left ideal in $A\left(\mathfrak{D}_{0}\right), \mathfrak{H} \cap A\left(\mathfrak{D}_{0}\right)=B$. Hence $\mathscr{H} \cap \mathfrak{O} \mathfrak{X}=B \cap \mathfrak{Q X}$. Similarly if $\mathcal{X}^{\prime}$ is the set of all $b \in \mathfrak{B}$ such that $\pi\left(b^{\prime}\right) \psi_{00}=0$ then $\mathscr{X}^{\prime} \supset \mathfrak{B} \mathfrak{N}\left(\mathfrak{D}_{0}\right)+\mathfrak{B} \mathfrak{Z}_{x}$ and $\mathfrak{X}^{\prime} \cap \mathfrak{O X}=B \cap \mathfrak{Q X}$. Therefore from Theorem 2, $\mathcal{X}$ and $\mathcal{X}^{\prime}$ are equivalent and so the representations $\gamma$ and $\pi$ are infinitesimally equivalent (see $[5, \S 10]$ ). 
We keep to the above notation. For any $\mathfrak{D}^{*} \in \Omega^{*}$ let $\mathfrak{S}_{\mathfrak{D}^{*}}^{*}$ denote the set of those elements in $\mathfrak{S}^{\prime}$ which transform under $\tau\left(K^{*}\right)$ according to $\mathfrak{D}^{*}(\tau$ is the left regular representation of $K^{*}$ on $\mathfrak{S}=L_{2}\left(K^{*}\right)$ ). Then it follows from the Frobenius reciprocity relation (Weil $[7$, p. 83]) and our construction of the element $f_{00}$ that $\operatorname{dim} \mathfrak{W}_{\mathfrak{D}^{*}}^{\prime} \leqq\left(\mathfrak{D}^{*}: \delta^{*}\right) d\left(\mathfrak{D}^{*}\right)$ where $\delta^{*}$ is the class in $\omega^{*}$ dual to $\delta^{*}$ and $\left(\mathfrak{D}^{*}: \delta^{*}\right)$ is the number of times $\delta^{*}$ occurs in the reduction of $\mathfrak{D}^{*}$ with respect to $M^{*}$. Since $\gamma$ and $\pi$ are infinitesimally equivalent we get $\operatorname{dim} V_{\mathfrak{D}} \leqq(\mathfrak{D}: \delta) d(\mathfrak{D})(\mathfrak{D} \in \Omega)$. Here $\delta$ is an equivalence class of finite-dimensional simple representations $\left.{ }^{4}\right)$ of $M$ defined as follows. If $\sigma^{*}$ is a representation in $\delta^{*}$ we define a representation $\sigma$ of class $\delta$ by $\sigma(m)=e^{-\mu(\Gamma(m))} \sigma^{*}\left(m^{*}\right)$ $(m \in M) .(\mathfrak{D}: \delta)$ is the number of times $\delta$ occurs in the reduction of $\mathfrak{D}$ with respect to $M$ (see Lemma 5 of [5]).

In particular since $\operatorname{dim} V_{\mathfrak{D}_{0}}>0,(\mathfrak{D}: \delta)>0$ and so there exists an element $\psi_{0} \in V_{\Phi_{0}}$ such that $\pi\left(X_{\alpha}\right) \psi_{0}=0\left(\alpha \in P_{-}\right)$and $\pi(H) \psi_{0}=\lambda_{\delta}(H) \psi_{0}\left(H \in \mathfrak{h}_{\mathfrak{f}}\right)$. Here $\lambda_{\delta}$ is the highest $\left(^{5}\right)$ weight of $\delta$. Let $z$ be an element in the center 8 of $\mathfrak{B}$. We know (Lemma 36 of [3]) that there exists a unique element $h$ in the subalgebra of $\mathfrak{B}$ generated by $(1, \mathfrak{h})$ such that $z-h \in \sum_{\alpha \in P} \mathfrak{B} X_{\alpha}$. Since $z$ and $h$ are both of rank zero it follows easily that $z-h \in \sum_{\alpha, \beta \in P_{+}} X_{-\alpha} \mathfrak{B} X_{\beta}$ $+\sum_{\alpha \in P_{-}} \mathfrak{B} X_{\alpha}$ (see proof of Lemma 9). Therefore if we extend the involution $\theta$ of $\mathfrak{g}$ (see $\$ 2$ of [5]) to an automorphism of $\mathfrak{B}$ we get

$$
\theta(z)-\theta(h) \in \sum_{\alpha, \beta \in P_{+}} X_{\alpha} \mathfrak{B} X_{-\beta}+\sum_{\alpha \in P_{-}} \mathfrak{B} X_{\alpha} .
$$

Hence in the notation of Lemma 11,

$$
\eta_{u, y}^{\mathfrak{D}_{0}^{*}}(\theta(z))=\overline{\beta_{y}\left(\xi_{u}(\theta(z))\right)}=\overline{\beta_{y}\left(\xi_{u}(\theta(h)+w)\right)}
$$

where $w \in \sum_{\alpha \in P_{-}} \mathfrak{B} X_{\alpha}$. Therefore

$$
\pi(\theta(z)) \psi_{0}=\pi\left(\xi_{\Lambda_{p}}(\theta(h)) \psi_{0},\right.
$$

since $\pi\left(X_{\alpha}\right) \psi_{0}=0\left(\alpha \in P_{-}\right)$. Let $\Lambda^{\prime \prime}$ be the linear function on $\mathfrak{h}$ which coincides with $-\left(\Lambda_{\mathfrak{p}}+2 \rho\right)$ on $\mathfrak{h}_{\mathfrak{p}}$ and with $\lambda_{\delta}$ on $\mathfrak{h}_{\mathfrak{q}}$. Similarly let $\Lambda^{\prime}$ be the linear function which is equal to $\Lambda_{\mathfrak{p}}$ on $\mathfrak{h}_{\mathfrak{p}}$ and to $\lambda_{\delta}$ on $\mathfrak{h}_{\mathfrak{q}}$. Then $\theta\left(\Lambda^{\prime \prime}+\rho\right)=\Lambda^{\prime}+\rho$ and one proves easily $\left(^{(6)}\right.$ that $\chi_{\Lambda^{\prime \prime}}(z)=\chi_{\Lambda^{\prime}}(\theta(z))$. Now it is clear that

$$
\pi\left(\xi_{\Lambda_{\mathfrak{p}}}(\theta(h))\right) \psi_{0}=\chi_{\Lambda^{\prime \prime}}(z) \psi_{0}=\chi_{\Lambda^{\prime}}(\theta(z)) \psi_{0} .
$$

Hence $\pi(\theta(z)) \psi_{0}=\chi_{\Lambda^{\prime}}(\theta(z)) \psi_{0}$. Since $\Lambda^{\prime}$ is equal to $\Lambda$ on $\mathfrak{h}_{\mathfrak{p}}$ we can replace $\Lambda$ by $\Lambda^{\prime}$ in Lemma 15 . Moreover $\Lambda^{\prime}$ coincides on $h_{t}$ with the highest weight $\lambda_{\delta}$ of an irreducible class $\delta$ of $M$ which has the property that $\operatorname{dim} V_{D}$ $\leqq d(\mathfrak{D})(\mathfrak{D}: \delta)$ for any $\mathfrak{D} \in \Omega$. Thus if we denote by $\omega$ the set of all equivalence

(4) $M$ is the analytic subgroup of $K$ corresponding to $\mathfrak{m}_{0}=\mathfrak{l}_{0} \cap\left(\mathfrak{h}_{\mathfrak{t}}+\sum_{\alpha \in P_{-}}\left(C X_{\alpha}+C X_{-\alpha}\right)\right)$.

(5) This means that if $\lambda$ is any other weight of $\delta\left(\lambda \neq \lambda_{\delta}\right)$ then $\lambda_{\delta}-\lambda>0$ (see $\$ 2$ of [5]).

(6) The proof depends on a calculation very similar to the one made in [3] on p. 79. 
classes of irreducible finite-dimensional representations of $M$ we get the following theorem.

THEOREM 4. Let $\pi$ be an irreducible quasi-simple representation of $\mathfrak{B}$ on a vector space $V$ such that some $\mathfrak{D}_{0} \in \Omega_{F}$ occurs in $\pi$. Then there exists a linear function $\Lambda$ on $\mathfrak{h}$ with the following properties:

(1) $\chi_{\Lambda}$ is the infinitesimal character of $\pi$,

(2) $\Lambda$ coincides on $\mathfrak{h}_{t}$ with the highest weight $\lambda_{\delta}$ of some $\delta \in \omega$,

(3) $\operatorname{dim} V_{\mathfrak{D}} \leqq d(\mathfrak{D})(\mathfrak{D}: \delta)$ for any $\mathfrak{D} \in \Omega$.

(4) $\pi$ is infinitesimally equivalent to a representation $\gamma$ of $G$ which is deducible $\left({ }^{7}\right)$ from $\pi_{\Lambda p, \mu}$ where $\mu$ is a suitable linear function on $\mathrm{c}$.

The above theorem contains some of the results announced in an earlier note (Theorems 1 and $2[4(\mathrm{~b})]$ ). The fourth assertion of Theorem 2 follows immediately if we take into account the definition of $\pi_{\Lambda p, \mu}$. For let $\mathfrak{S}=L_{2}\left(K^{*}\right)$ be the Hilbert space introduced above and let $\mathfrak{D}_{1}, \mathfrak{D}_{2}$ be two classes in $\Omega$ which occur in $\pi_{\Lambda p, \mu}$. Let $g_{i} \in \mathfrak{S}_{\mathfrak{D}_{i}}(i=1,2)$ and consider the scalar product $\left(g_{1}, \pi_{\Lambda p, \mu}(x) g_{2}\right)$ in $\mathfrak{S}$. Then if a bar denotes complex conjugate,

$$
\begin{aligned}
\left(g_{1}, \pi_{\Lambda_{\mathfrak{p}}, \mu}(x) g_{2}\right) & =\int_{K^{*}} \overline{g_{1}\left(u^{*}\right)} e^{-\mu\left(\Gamma\left(x^{-1}, u^{*}\right)\right)} e^{-\left(\Lambda_{p}+2 \rho\right)\left(H\left(x^{-1}, u^{*}\right)\right)} g_{2}\left(u_{x^{-1}}^{*}\right) d u^{*} \\
& =\int_{K^{*}} \overline{g_{1}\left(u_{x}^{*}\right) e^{\mu\left(\Gamma\left(x, u^{*}\right)\right)}} e^{\Lambda_{\mathfrak{p}}\left(H\left(x, u^{*}\right)\right)} g_{2}\left(u^{*}\right) d u^{*}
\end{aligned}
$$

if we recall that $d u^{*}=e^{2 \rho\left(H\left(x, u^{*}\right)\right)} d u_{x}^{*}($ see $[5, \S 11])$. Put

$$
\sigma_{i}^{*}\left(u^{*}\right)=e^{-\mu(\Gamma(u))} \sigma_{i}(u) \quad(u \in K)(i=1,2)
$$

where $\sigma_{i}$ is some representation $\mathfrak{D}_{i}$. Then $\sigma_{i}{ }^{*}$ is a representation of $K^{*}$ which we may assume to be unitary. The complex conjugates of the matrix coefficients of $\sigma_{i}^{*}$ span the space $\mathfrak{S}_{\mathfrak{D}_{i}}$. Hence $g_{i}$ is a linear combination of the complex conjugates of these functions. Let $E_{\mathbb{D}_{\mathfrak{i}}}$ be the orthogonal projection of $\mathfrak{S}$ on $\mathfrak{S D}_{\mathfrak{D}_{i}}$. Then it follows that the matrix coefficients of $E_{\mathfrak{D}_{1}} \pi_{\Delta p, \mu}(x) E_{\mathfrak{D}_{2}}$ are finite linear combinations of functions of the form

$$
\int_{K^{*}} g_{1}\left(u_{x}^{*}\right) e^{\mu\left(\Gamma\left(x, u^{*}\right)\right)} e^{\Delta_{p}\left(H\left(x, u^{*}\right)\right)} g_{2}\left(u^{*-1}\right) d u^{*}
$$

where $g_{i}$ is a matrix coefficient of some representation in $\mathfrak{D}_{i}{ }^{*}$. Since $\gamma$ is deducible from $\pi_{\Lambda p, \mu}$ the same holds for the matrix coefficients for $E_{\mathfrak{D}_{1}}^{\prime} \gamma(x) E_{\mathfrak{D}_{2}}^{\prime}$ where $E_{\mathfrak{D}_{i}}^{\prime}$ is the orthogonal projection of the representation space $\mathfrak{S}^{\prime}$ of $\gamma$ on the subspace $\mathfrak{S}_{\mathfrak{D}_{i}}^{\prime}$ corresponding to $\mathfrak{D}_{i}(i=1,2)$. These matrix coefficients

$\left({ }^{7}\right)$ Let $\sigma$ be a representation of $G$ on a Hilbert space $\mathfrak{W}$. Let $\mathfrak{W}_{1}, \mathfrak{W}_{2}$ be two closed invariant subspaces of $\mathfrak{S}$ such that $\mathfrak{W}_{1}>\mathfrak{W}_{2}$. We regard the factor space $\mathfrak{W}_{1} / \mathfrak{W}_{2}$ as a Hilbert space in the usual way and consider the representation $\sigma^{\prime}$ of $G$ induced on it under $\sigma$. Any representation $\sigma^{\prime}$ obtained in this fashion is said to be deducible from $\sigma$ (see [4(b)]. 
are analytic functions (see $[5, \S 6]$ ) on $G$ and so they are completely determined as soon as we know all their partial derivatives at $x=1$. Since $\gamma$ is infinitesimally equivalent to $\pi$, it follows that the values of these derivatives at $x=1$ coincide with the values of the corresponding derivatives of the coefficients of $\pi(x)$ computed with respect to suitable bases in $V_{\mathfrak{D}_{i}}(i=1,2)$. Hence the two sets of matrix coefficients for $\gamma(x)$ and $\pi(x)$ are identical as analytic functions on $G$. In view of the above result for $\gamma$ the fourth assertion of Theorem 2 of [4(b)] now follows for $\pi$.

In particular if $\sigma_{1}{ }^{*}, \sigma_{2}{ }^{*}$ are both trivial representations of $K^{*}$, the corresponding matrix coefficient of $\pi(x)$ is a numerical multiple of $\int_{K^{*}} e^{\mu\left(\Gamma\left(x, u^{*}\right)\right)} e^{\Lambda \mathfrak{p}\left(H\left(x, u^{*}\right)\right)} d u^{*}$. However since the value of this coefficient for $x=1$ is 1 the multiplicative constant involved must be 1 .

THEOREM( $\left.{ }^{8}\right)$ 5. Let $\pi$ be a quasi-simple irreducible representation of $G$ on a Banach space $\mathfrak{S}$. Let $\mathfrak{D}_{0}$ be a class in $\Omega$ which occurs in $\pi$ and such that $d\left(\mathfrak{D}_{0}\right)=1$. Then $\operatorname{dim} \mathfrak{S}_{\mathfrak{D}_{0}}=1$. Choose $\psi \in \mathfrak{S}_{\mathfrak{D}_{0}}(\psi \neq 0)$ and let $\mu$ be the linear function on $\mathfrak{l}$ such that $\pi(X) \psi=\mu(X) \psi(X \in \mathfrak{f})$. Then there exists a linear function $\Lambda$ on $\mathfrak{h}$ such that the following conditions are fulfilled:

(1) $\Lambda$ coincides with $\mu$ on $\mathfrak{h}_{t}$ and $\chi_{\Lambda}$ is the infinitesimal character of $\pi$.

(2) If $E_{\mathfrak{D}_{0}}$ is the canonical projection of $\mathfrak{S}$ on $\mathfrak{S E D}_{\mathbb{D}}($ see $[5, \S 8])$ then

$$
E_{D_{0}} \pi(x) \psi=\int_{K^{*}} e^{\mu\left(\Gamma\left(x, u^{*}\right)\right)} e^{\Lambda\left(H\left(x, u^{*}\right)\right)} d u^{*} \psi \quad(x \in G) .
$$

Since $d\left(\mathfrak{D}_{0}\right)=1$ every representation $\sigma \in \mathfrak{D}_{0}$ is abelian and therefore $\sigma\left(\mathfrak{f}^{\prime}\right)$ $=\{0\}$. Hence $\mathfrak{D}_{0} \in \Omega_{F}$ and Theorem 3 is applicable. Choose $\Lambda$ in accordance with Theorem 3 . Then $\operatorname{dim} \mathfrak{S}_{\mathfrak{D}_{0}} \leqq d\left(\mathfrak{D}_{0}\right)\left(\mathfrak{D}_{0}: \delta\right) \leqq d\left(\mathfrak{D}_{0}\right)^{2}=1$. Since $\mathfrak{D}_{0}$ occurs in

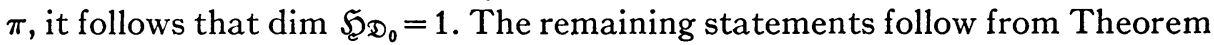
3 and what we have said above.

5. Appendix. The following lemma is frequently useful.

Lemma 16. Let $\mathcal{A}$ be an associate algebra with 1 over a field $K$ of characteristic zero and let $\pi_{1}, \cdots, \pi_{r}\left(\pi_{j} \neq 0,1 \leqq j \leqq r\right)$ be a finite number of finite-dimensional irreducible representations of $\mathcal{A}$ no two of which are equivalent. Put $s_{j}(a)$ $=\operatorname{sp} \pi_{j}(a), 1 \leqq j \leqq r(a \in \mathcal{A})$. Then the linear functions $s_{1}, \cdots, s_{r}$ on $A$ are linearly independent over $K$.

Let $\pi$ be the direct sum of the representations $\pi_{1}, \cdots, \pi_{r}$. Then $\mathcal{A}^{\prime}$ $=\pi(\mathcal{A})$ is a finite-dimensional semisimple algebra. Let $\mathfrak{N}_{j}$ be the kernel of $\pi_{j}$. Since $\pi(a) \rightarrow \pi_{j}(a)(a \in \mathcal{A})$ is an irreducible representation of $\mathcal{A}^{\prime}, \mathfrak{N}_{j}^{\prime}$ $=\pi\left(\mathfrak{N}_{j}\right)$ is a maximal two-sided ideal in $\mathcal{A}^{\prime}$. Since $\mathcal{A}^{\prime}$ is semisimple there exists a two-sided ideal $\mathcal{A}_{j}^{\prime}$ in $\mathcal{A}^{\prime}$ which is complementary to $\mathfrak{R}_{j}^{\prime}$. It is clear that $\mathcal{A}_{j}^{\prime}$ is a simple ideal of $\mathcal{A}^{\prime}$. Now if $k \neq j, \pi_{k}$ is not equivalent to $\pi_{j}$ and therefore $\mathfrak{N}_{k}^{\prime} \neq \mathfrak{N}_{j}^{\prime}$. Hence $\mathcal{A}_{j}^{\prime} \neq \mathcal{A}_{k}^{\prime}$ and therefore $\mathcal{A}_{1}^{\prime}, \cdots, \mathcal{A}_{r}^{\prime}$ are distinct

$(8)$ This result was announced in $[4(a)]$. 
minimal ideals in $\mathcal{A}^{\prime}$. But $\mathcal{A}^{\prime}$ is the direct.sum of its simple ideals and therefore $\Re_{j} \supset \mathcal{A}_{k}^{\prime}(k \neq j)$. Choose $e_{j} \in \mathcal{A}$ such that $\pi\left(e_{j}\right)$ is the unit element of the simple algebra $\mathcal{A}_{j}^{\prime}$. Then since $\pi\left(e_{j}\right) \in \mathfrak{R}_{k}^{\prime}(k \neq j), \pi_{k}\left(e_{j}\right)=0$. On the other hand $\pi\left(1-e_{j}\right) \in \mathfrak{N}_{j}^{\prime}$ and so $\pi_{j}\left(1-e_{j}\right)=0$. This shows that $s_{k}\left(e_{j}\right)=0$ if $k \neq j$ and $s_{j}\left(e_{j}\right)=d_{j}$ where $d_{j}$ is the degree of $\pi_{j}$. The assertion of the lemma is now obvious.

\section{REFERENCES}

1. E. Cartan, Ann. École Norm. vol. 44 (1927).

2. Harish-Chandra, Ann. of Math. vol. 50 (1949) pp. 900-915.

3. - Trans. Amer. Math. Soc. vol. 70 (1951) pp. 28-96.

4. - (a) Proc. Nat. Acad. Sci. U.S.A. vol. 37 (1951) pp. 362-365. (b) Proc. Nat. Acad. Sci. U.S.A. vol. 37 (1951) pp. 691-694.

5. - Representation of a semisimple Lie group on a Banach space. I, Trans. Amer. Math. Soc. vol. 75 (1953) pp. 185-243.

6. G. D. Mostow, Bull. Amer. Math. Soc. vol. 55 (1949) pp. 969-980. 1940.

7. A. Weil, L'integration dans les groupes topologiques et ses applications, Paris, Hermann,

8. H. Weyl, Math. Zeit. vol. 24 (1925) pp. 328-395.

Columbia University,

NEW YORK, N. Y.

TATA Institute of Fundamental REsearch, Bombay, INDIA. 